\title{
Interferometer Techniques for Gravitational-Wave Detection
}

\author{
Andreas Freise \\ School of Physics and Astronomy \\ University of Birmingham \\ Birmingham, B15 2TT, UK \\ email: adf@star.sr.bham.ac.uk \\ http://www .gwoptics.org \\ Kenneth Strain \\ Department of Physics and Astronomy \\ University of Glasgow \\ Glasgow G12 8QQ, UK \\ email: k.strain@physics.gla.ac.uk
}

Accepted on 15 February 2010

Published on 25 February 2010

\begin{abstract}
Several km-scale gravitational-wave detectors have been constructed world wide. These instruments combine a number of advanced technologies to push the limits of precision length measurement. The core devices are laser interferometers of a new kind; developed from the classical Michelson topology these interferometers integrate additional optical elements, which significantly change the properties of the optical system. Much of the design and analysis of these laser interferometers can be performed using well-known classical optical techniques, however, the complex optical layouts provide a new challenge. In this review we give a textbook-style introduction to the optical science required for the understanding of modern gravitational wave detectors, as well as other high-precision laser interferometers. In addition, we provide a number of examples for a freely available interferometer simulation software and encourage the reader to use these examples to gain hands-on experience with the discussed optical methods.
\end{abstract}

This review is licensed under a Creative Commons Attribution-Non-Commercial-NoDerivs 3.0 Germany License. http://creativecommons.org/licenses/by-nc-nd/3.0/de/ 


\section{Imprint / Terms of Use}

Living Reviews in Relativity is a peer reviewed open access journal published by the Max Planck Institute for Gravitational Physics, Am Mühlenberg 1, 14476 Potsdam, Germany. ISSN 1433-8351.

This review is licensed under a Creative Commons Attribution-Non-Commercial-NoDerivs 3.0 Germany License: http://creativecommons.org/licenses/by-nc-nd/3.0/de/

Because a Living Reviews article can evolve over time, we recommend to cite the article as follows:

Andreas Freise and Kenneth Strain, "Interferometer Techniques for Gravitational-Wave Detection", Living Rev. Relativity, 13, (2010), 1. [Online Article]: cited [<date $>$ ], http://www.livingreviews.org/lrr-2010-1

The date given as $<$ date $>$ then uniquely identifies the version of the article you are referring to.

\section{Article Revisions}

Living Reviews supports two different ways to keep its articles up-to-date:

Fast-track revision A fast-track revision provides the author with the opportunity to add short notices of current research results, trends and developments, or important publications to the article. A fast-track revision is refereed by the responsible subject editor. If an article has undergone a fast-track revision, a summary of changes will be listed here.

Major update A major update will include substantial changes and additions and is subject to full external refereeing. It is published with a new publication number.

For detailed documentation of an article's evolution, please refer always to the history document of the article's online version at http://www.livingreviews.org/lrr-2010-1. 


\section{Contents}

1 Introduction $\quad \mathbf{5}$

1.1 The scope and style of the review . . . . . . . . . . . . . . . . . . . . . . . .

1.2 Overview of the goals of interferometer design . . . . . . . . . . . . . . . . . . . 5

1.3 Overview of the physics of the primary interferometer components . . . . . . . 6

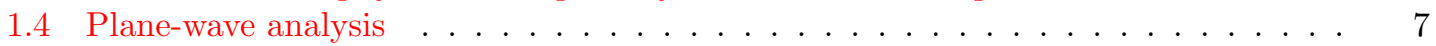

1.5 Frequency domain analysis . . . . . . . . . . . . . . . . . 8

2 Optical Components: Coupling of Field Amplitudes 9

2.1 Mirrors and spaces: reflection, transmission and propagation . . . . . . . . . 9

2.2 The two-mirror resonator . . . . . . . . . . . . . . . . . . . . 10

2.3 Coupling matrices . . . . . . . . . . . . . . . . . . . . . . . . . . . . . . . . . . . . . . . . .

2.4 Phase relation at a mirror or beam splitter . . . . . . . . . . . . . . . . 13

2.4.1 Composite optical surfaces . . . . . . . . . . . . . . . . . . 14

2.5 Lengths and tunings: numerical accuracy of distances . . . . . . . . . . . . . . . . 17

2.6 Revised coupling matrices for space and mirrors . . . . . . . . . . . . . . . . . . 19

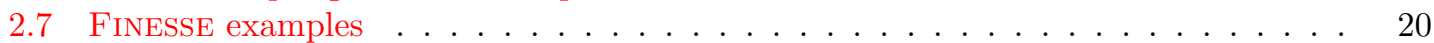

2.7.1 Mirror reflectivity and transmittance . . . . . . . . . . . . . . . . 20

2.7 .2 Length and tunings . . . . . . . . . . . . . . . . . . 20

3 Light with Multiple Frequency Components 2

3.1 Modulation of light fields . . . . . . . . . . . . . . . . . . . . . . . . . . . . . . . . . . . . . . .

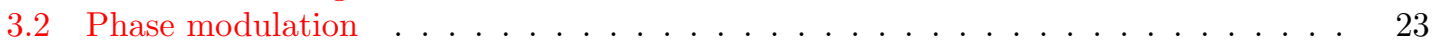

3.3 Frequency modulation . . . . . . . . . . . . . . . . . . . . . . . . . . . . . . . . . . . . .

3.4 Amplitude modulation . . . . . . . . . . . . . . . . . . . . . . . 25

3.5 Sidebands as phasors in a rotating frame . . . . . . . . . . . . . . . . . . 25

3.6 Phase modulation through a moving mirror . . . . . . . . . . . . . . . . 27

3.7 Coupling matrices for beams with multiple frequency components . . . . . . . . . 28

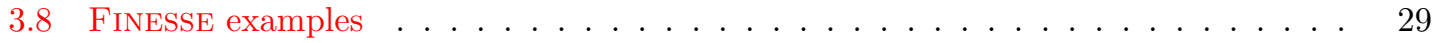

3.8.1 Modulation index . . . . . . . . . . . . . . . . . . . . . . . . . . . . . . . . . . . . .

3.8.2 Mirror modulation . . . . . . . . . . . . . . . . . . 29

4 Optical Readout $\quad 31$

4.1 Detection of optical beats . . . . . . . . . . . . . . . . . . . . . . . . . . . . . . . .

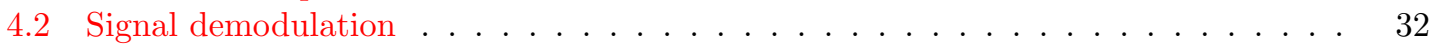

4.3 Finesse examples . . . . . . . . . . . . . . . . . . . . . . . . . . . . . . . . . . . . . . . . .

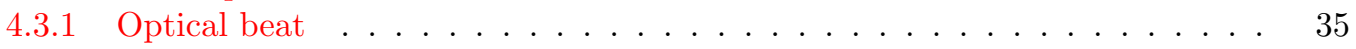

$5 \quad$ Basic Interferometers $\quad \mathbf{3 6}$

5.1 The two-mirror cavity: a Fabry-Pérot interferometer . . . . . . . . . . . . . . 36

5.2 Michelson interferometer . . . . . . . . . . . . . . . . . . . . . 40

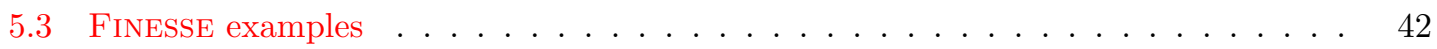

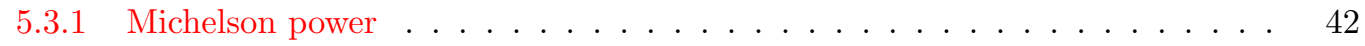

5.3.2 Michelson modulation . . . . . . . . . . . . . . . . 42

6 Interferometric Length Sensing and Control $\quad 44$

6.1 Error signals and transfer functions . . . . . . . . . . . . . . . . . . . . 44

6.2 Fabry-Pérot length sensing . . . . . . . . . . . . . . . . . . . . 46

6.3 The Pound-Drever-Hall length sensing scheme . . . . . . . . . . . . . . . . . . . . 47

6.4 Michelson length sensing . . . . . . . . . . . . . . . . . . . . . . 48 
6.5 The Schnupp modulation scheme . . . . . . . . . . . . . . . . . 50

6.6 Finesse examples . . . . . . . . . . . . . . . . . . . . . 51

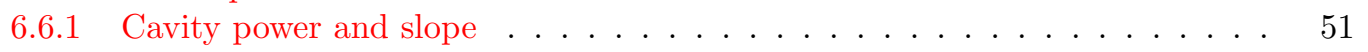

6.6.2 Michelson with Schnupp modulation . . . . . . . . . . . . . . 51

7 Beam Shapes: Beyond the Plane Wave Approximation 53

7.1 The paraxial wave equation . . . . . . . . . . . . . . . . 53

7.2 Transverse electromagnetic modes . . . . . . . . . . . . . . . . . . 54

7.3 Properties of Gaussian beams . . . . . . . . . . . . . . . . . . . . . 55

7.4 Astigmatic beams: the tangential and sagittal plane . . . . . . . . . . . . . 57

7.5 Higher-order Hermite-Gauss modes . . . . . . . . . . . . . . . . . . . . . . . 57

7.6 The Gaussian beam parameter . . . . . . . . . . . . . . . . . . . . . 58

7.7 Properties of higher-order Hermite-Gauss modes . . . . . . . . . . . . . . . . 59

7.8 Gouy phase . . . . . . . . . . . . . . . . . . . . . . . . . . . . . . . . . . . . . . . .

7.9 Laguerre-Gauss modes . . . . . . . . . . . . . . . . . . . . . . . . . . . . . . . . . . . . . 62

7.10 Tracing a Gaussian beam through an optical system . . . . . . . . . . . . . . . 64

7.11 ABCD matrices . . . . . . . . . . . . . . . . . . . 66

8 Interferometer Matrix with Hermite-Gauss Modes $\quad 69$

8.1 Coupling of Hermite-Gauss modes . . . . . . . . . . . . . . . . . . . . 69

8.2 Coupling coefficients for Hermite-Gauss modes . . . . . . . . . . . . . . . . . 70

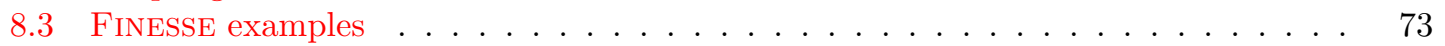

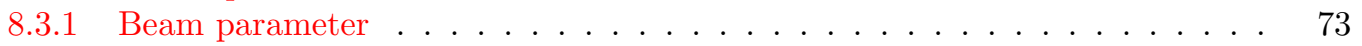

8.3 .2 Mode cleaner . . . . . . . . . . . . . . . . . . . . . . 73

8.3 .3 LG33 mode . . . . . . . . . . . . . . . . . . . . . 74

9 Acknowledgements $\quad 76$

$\begin{array}{ll}\text { A The Interferometer Simulation Finesse } & \mathbf{7 6}\end{array}$

$\begin{array}{lr}\text { References } & \mathbf{7 7}\end{array}$ 


\section{Introduction}

\subsection{The scope and style of the review}

The historical development of laser interferometers for application as gravitational-wave detectors [47] has involved the combination of relatively simple optical subsystems into more and more complex assemblies. The individual elements that compose the interferometers, including mirrors, beam splitters, lasers, modulators, various polarising optics, photo detectors and so forth, are individually well described by relatively simple, mostly-classical physics. Complexity arises from the combination of multiple mirrors, beam splitters etc. into optical cavity systems, have narrow resonant features, and the consequent requirement to stabilise relative separations of the various components to sub-wavelength accuracy, and indeed in many cases to very small fractions of a wavelength.

Thus, classical physics describes the interferometer techniques and the operation of current gravitational-wave detectors. However, we note that at signal frequencies above a couple of hundreds of Hertz, the sensitivity of current detectors is limited by the photon counting noise at the interferometer readout, also called shot-noise. The next generation systems such as Advanced LIGO [23, 5], Advanced Virgo [4] and LCGT [36] are expected to operate in a regime where the quantum physics of both light and mirror motion couple to each other. Then, a rigorous quantummechanical description is certainly required. Sensitivity improvements beyond these 'Advanced' detectors necessitate the development of non-classical techniques. The present review, in its first version, does not consider quantum effects but reserves them for future updates.

The components employed tend to behave in a linear fashion with respect to the optical field, i.e., nonlinear optical effects need hardly be considered. Indeed, almost all aspects of the design of laser interferometers are dealt with in the linear regime. Therefore the underlying mathematics is relatively simple and many standard techniques are available, including those that naturally allow numerical solution by computer models. Such computer models are in fact necessary as the exact solutions can become quite complicated even for systems of a few components. In practice, workers in the field rarely calculate the behaviour of the optical systems from first principles, but instead rely on various well-established numerical modelling techniques. An example of software that enables modelling of either time-dependent or frequency-domain behaviour of interferometers and their component systems is FInESsE [22, 19]. This was developed by one of us (AF), has been validated in a wide range of situations, and was used to prepare the examples included in the present review.

The target readership we have in mind is the student or researcher who desires to get to grips with practical issues in the design of interferometers or component parts thereof. For that reason, this review consists of sections covering the basic physics and approaches to simulation, intermixed with some practical examples. To make this as useful as possible, the examples are intended to be realistic with sensible parameters reflecting typical application in gravitational wave detectors. The examples, prepared using Finesse, are designed to illustrate the methods typically applied in designing gravitational wave detectors. We encourage the reader to obtain FinessE and to follow the examples (see Appendix A).

\subsection{Overview of the goals of interferometer design}

As set out in very many works, gravitational-wave detectors strive to pick out signals carried by passing gravitational waves from a background of self-generated noise. The principles of operation are set out at various points in the review, but in essence, the goal has been to prepare many photons, stored for as long as practical in the 'arms' of a laser interferometer (traditionally the two arms are at right angles), so that tiny phase shifts induced by the gravitational waves form 
as large as possible a signal, when the light leaving the appropriate 'port' of the interferometer is detected and the resulting signal analysed.

The evolution of gravitational-wave detectors can be seen by following their development from prototypes and early observing systems towards the Advanced detectors, which are currently in the final stages of planning or early stages of construction. Starting from the simplest Michelson interferometer [18], then by the application of techniques to increase the number of photons stored in the arms: delay lines [31], Fabry-Pérot arm cavities [16, 17] and power recycling [15]. The final step in the development of classical interferometry was the inclusion of signal recycling [41, 30], which, among other effects, allows the signal from a gravitational-wave signal of approximatelyknown spectrum to be enhanced above the noise.

Reading out a signal from even the most basic interferometer requires minimising the coupling of local environmental effects to the detected output. Thus, the relative positions of all the components must be stabilised. This is commonly achieved by suspending the mirrors etc. as pendulums, often multi-stage pendulums in series, and then applying closed-loop control to maintain the desired operating condition. The careful engineering required to provide low-noise suspensions with the correct vibration isolation, and also low-noise actuation, is described in many works. As the interferometer optics become more complicated, the resonance conditions, i.e., the allowed combinations of inter-component path lengths required to allow the photon number in the interferometer arms to reach maximum, become more narrowly defined. It is likewise necessary to maintain angular alignment of all components, such that beams required to interfere are correctly co-aligned. Typically the beams need to be aligned within a small fraction (and sometimes a very small fraction) of the far-field diffraction angle, and the requirement can be in the low nanoradian range for km-scale detectors [44, 21]. Therefore, for each optical component there is typically one longitudinal (i.e., along the direction of light propagation), plus two angular degrees of freedom (pitch and yaw about the longitudinal axis). A complex interferometer can consist of up to around seven highly sensitive components and so there can be of order 20 degrees of freedom to be measured and controlled [3, 57].

Although the light fields are linear, the coupling between the position of a mirror and the complex amplitude of the detected light field typically shows strongly nonlinear dependence on mirror positions due to the sharp resonance features exhibited by cavity systems. However, the fields do vary linearly or at least smoothly close to the desired operating point. So, while wellunderstood linear control theory suffices to design the control system needed to maintain the optical configuration at its operating point, bringing the system to that operating condition is often a separate and more challenging nonlinear problem. In the current version of this work we consider only the linear aspects of sensing and control.

Control systems require actuators, and those employed are typically electrical-force transducers that act on the suspended optical components, either directly or - to provide enhanced noise rejection - at upper stages of multi-stage suspensions. The transducers are normally coil-magnet actuators, with the magnets on the moving part, or, less frequently, electrostatic actuators of varying design. The actuators are frequently regarded as part of the mirror suspension subsystem and are not discussed in the current work.

\subsection{Overview of the physics of the primary interferometer components}

To give order to our review we consider the main physics describing the operation of the basic optical components (mirrors, beam splitters, modulators, etc.) required to construct interferometers. Although all of the relevant physics is generally well known and not new, we take it as a starting point that permits the introduction of notation and conventions. It is also true that the interferometry employed for gravitational-wave detection has a different emphasis than other interferometer applications. As a consequence, descriptions or examples of a number of crucial optical

Living Reviews in Relativity

http: //www . livingreviews . org/lrr-2010-1 
properties for gravitational wave detectors cannot be found in the literature. The purpose of this first version of the review is especially to provide a coherent theoretical framework for describing such effects. With the basics established, it can be seen that the interferometer configurations that have been employed in gravitational-wave detection may be built up and simulated in a relatively straightforward manner.

As mentioned above, we do not address the newer physics associated with operation at or beyond the standard quantum limit. The interested reader can begin to explore this topic from the following references.

- The standard quantum limit $[10,32]$

- Squeezing $[38,53]$

- Quantum nondemolition interferometry [9, 24]

These matters are to be included in a future revision of this review.

\subsection{Plane-wave analysis}

The main optical systems of interferometric gravitational-wave detectors are designed such that all system parameters are well known and stable over time. The stability is achieved through a mixture of passive isolation systems and active feedback control. In particular, the light sources are some of the most stable, low-noise continuous-wave laser systems so that electromagnetic fields can be assumed to be essentially monochromatic. Additional frequency components can be modelled as small modulations (in amplitude or phase). The laser beams are well collimated, propagate along a well-defined optical axis and remain always very much smaller than the optical elements they interact with. Therefore, these beams can be described as paraxial and the well-known paraxial approximations can be applied.

It is useful to first derive a mathematical model based on monochromatic, scalar, plane waves. As it turns out, a more detailed model including the polarisation and the shape of the laser beam as well as multiple frequency components, can be derived as an extension to the plane-wave model. A plane electromagnetic wave is typically described by its electric field component:

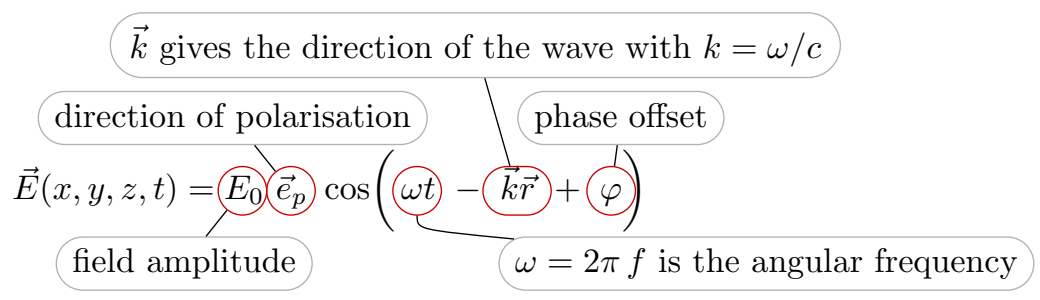

Figure 1

with $E_{0}$ as the (constant) field amplitude in $\mathrm{V} / \mathrm{m}, \vec{e}_{p}$ the unit vector in the direction of polarisation, such as, for example, $\vec{e}_{y}$ for $\mathscr{S}$-polarised light, $\omega$ the angular oscillation frequency of the wave, and $\vec{k}=\vec{e}_{k} \omega / c$ the wave vector pointing the in the direction of propagation. The absolute phase $\varphi$ only becomes meaningful when the field is superposed with other light fields.

In this document we will consider waves propagating along the optical axis given by the $z$-axis, so that $\vec{k} \vec{r}=k z$. For the moment we will ignore the polarisation and use scalar waves, which can be written as

$$
E(z, t)=E_{0} \cos (\omega t-k z+\varphi) .
$$


Further, in this document we use complex notation, i.e.,

$$
E=\Re\left\{E^{\prime}\right\} \quad \text { with } \quad E^{\prime}=E_{0}^{\prime} \exp (\mathrm{i}(\omega t-k z)) .
$$

This has the advantage that the scalar amplitude and the phase $\varphi$ can be given by one, now complex, amplitude $E_{0}^{\prime}=E_{0} \exp (\mathrm{i} \varphi)$. We will use this notation with complex numbers throughout. For clarity we will simply use the unprimed letters for the auxiliary field. In particular, we will use the letter $E$ and also $a$ and $b$ to denote complex electric-field amplitudes. But remember that, for example, in $E=E_{0} \exp (-\mathrm{i} k z)$ neither $E$ nor $E_{0}$ are physical quantities. Only the real part of $E$ exists and deserves the name field amplitude.

\subsection{Frequency domain analysis}

In most cases we are either interested in the fields at one particular location, for example, on the surface of an optical element, or we want to know the fields at all places in the interferometer but at one particular point in time. The latter is usually true for the steady state approach: assuming that the interferometer is in a steady state, all solutions must be independent of time so that we can perform all computations at $t=0$ without loss of generality. In that case, the scalar plane wave can be written as

$$
E=E_{0} \exp (-\mathrm{i} k z)
$$

The frequency domain is of special interest as numerical models of gravitational-wave detectors tend to be much faster to compute in the frequency domain than in the time domain. 


\section{Optical Components: Coupling of Field Amplitudes}

When an electromagnetic wave interacts with an optical system, all of its parameters can be changed as a result. Typically optical components are designed such that, ideally, they only affect one of the parameters, i.e., either the amplitude or the polarisation or the shape. Therefore, it is convenient to derive separate descriptions concerning each parameter. This section introduces the coupling of the complex field amplitude at optical components. Typically, the optical components are described in the simplest possible way, as illustrated by the use of abstract schematics such as those shown in Figure 2.

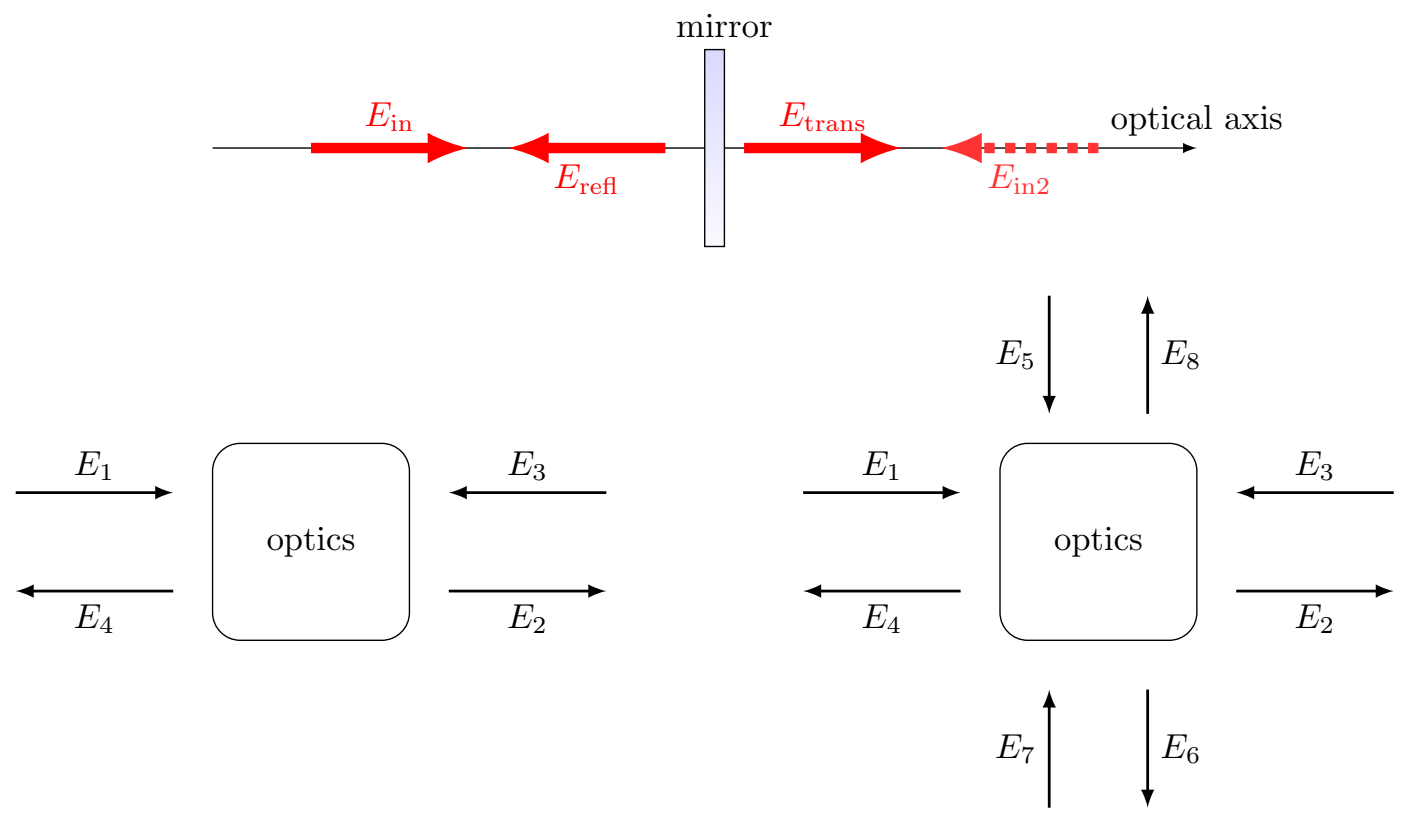

Figure 2: This set of figures introduces an abstract form of illustration, which will be used in this document. The top figure shows a typical example taken from the analysis of an optical system: an incident field $E_{\text {in }}$ is reflected and transmitted by a semi-transparent mirror; there might be the possibility of second incident field $E_{i n 2}$. The lower left figure shows the abstract form we choose to represent the same system. The lower right figure depicts how this can be extended to include a beam splitter object, which connects two optical axes.

\subsection{Mirrors and spaces: reflection, transmission and propagation}

The core optical systems of current interferometric gravitational interferometers are composed of two building blocks: a) resonant optical cavities, such as Fabry-Pérot resonators, and b) beam splitters, as in a Michelson interferometer. In other words, the laser beam is either propagated through a vacuum system or interacts with a partially-reflecting optical surface.

The term optical surface generally refers to a boundary between two media with possibly different indices of refraction $n$, for example, the boundary between air and glass or between two types of glass. A real fused silica mirror in an interferometer features two surfaces, which interact with a reflected or transmitted laser beam. However, in some cases, one of these surfaces has been treated with an anti-reflection (AR) coating to minimise the effect on the transmitted beam.

The terms mirror and beam splitter are sometimes used to describe a (theoretical) optical surface in a model. We define real amplitude coefficients for reflection and transmission $r$ and $t$, 
with $0 \leq r, t \leq 1$, so that the field amplitudes can be written as

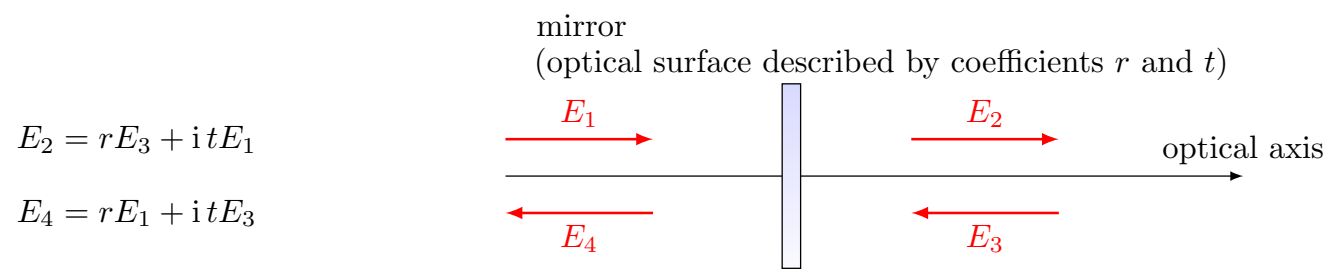

Figure 3

The $\pi / 2$ phase shift upon transmission (here given by the factor i) refers to a phase convention explained in Section 2.4.

The free propagation of a distance $D$ through a medium with index of refraction $n$ can be described with the following set of equations:

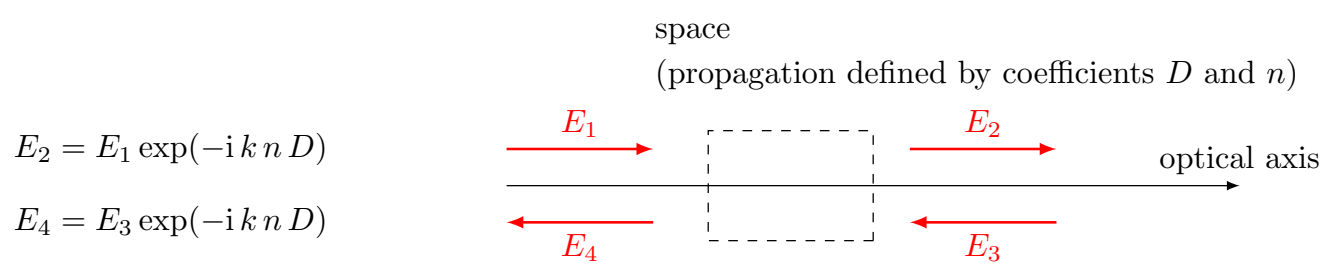

Figure 4

In the following we use $n=1$ for simplicity.

Note that we use above relations to demonstrate various mathematical methods for the analysis of optical systems. However, refined versions of the coupling equations for optical components, including those for spaces and mirrors, are also required, see, for example, Section 2.6.

\subsection{The two-mirror resonator}

The linear optical resonator, also called a cavity is formed by two partially-transparent mirrors, arranged in parallel as shown in Figure 5. This simple setup makes a very good example with which to illustrate how a mathematical model of an interferometer can be derived, using the equations introduced in Section 2.1.

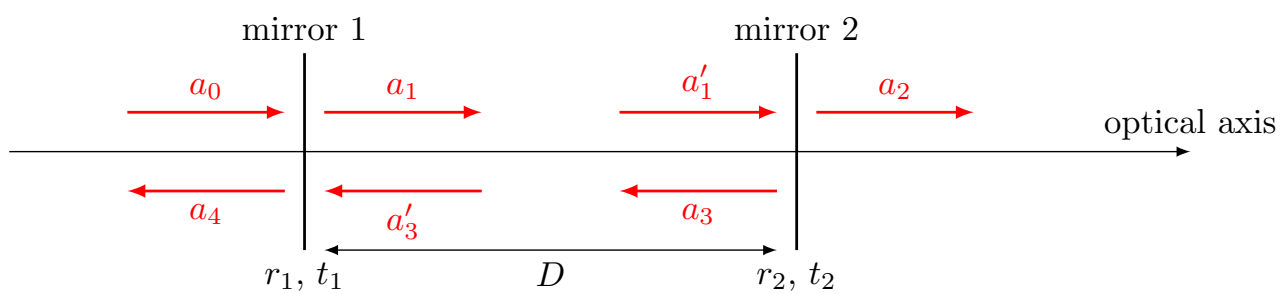

Figure 5: Simplified schematic of a two mirror cavity. The two mirrors are defined by the amplitude coefficients for reflection and transmission. Further, the resulting cavity is characterised by its length $D$. Light field amplitudes are shown and identified by a variable name, where necessary to permit their mutual coupling to be computed. 
The cavity is defined by a propagation length $D$ (in vacuum), the amplitude reflectivities $r_{1}$, $r_{2}$ and the amplitude transmittances $t_{1}, t_{2}$. The amplitude at each point in the cavity can be computed simply as the superposition of fields. The entire set of equations can be written as

$$
\begin{aligned}
& a_{1}=\mathrm{i} t_{1} a_{0}+r_{1} a_{3}^{\prime} \\
& a_{1}^{\prime}=\exp (-\mathrm{i} k D) a_{1} \\
& a_{2}=\mathrm{i} t_{2} a_{1}^{\prime} \\
& a_{3}=r_{2} a_{1}^{\prime} \\
& a_{3}^{\prime}=\exp (-\mathrm{i} k D) a_{3} \\
& a_{4}=r_{1} a_{0}+\mathrm{i} t_{1} a_{3}^{\prime}
\end{aligned}
$$

The circulating field impinging on the first mirror (surface) $a_{3}^{\prime}$ can now be computed as

$$
\begin{aligned}
a_{3}^{\prime} & =\exp (-\mathrm{i} k D) a_{3}=\exp (-\mathrm{i} k D) r_{2} a_{1}^{\prime}=\exp (-\mathrm{i} 2 k D) r_{2} a_{1} \\
& =\exp (-\mathrm{i} 2 k D) r_{2}\left(\mathrm{i} t_{1} a_{0}+r_{1} a_{3}^{\prime}\right)
\end{aligned}
$$

This then yields

$$
a_{3}^{\prime}=a_{0} \frac{\mathrm{i} r_{2} t_{1} \exp (-\mathrm{i} 2 k D)}{1-r_{1} r_{2} \exp (-\mathrm{i} 2 k D)} .
$$

We can directly compute the reflected field to be

$$
a_{4}=a_{0}\left(r_{1}-\frac{r_{2} t_{1}^{2} \exp (-\mathrm{i} 2 k D)}{1-r_{1} r_{2} \exp (-\mathrm{i} 2 k D)}\right)=a_{0}\left(\frac{r_{1}-r_{2}\left(r_{1}^{2}+t_{1}^{2}\right) \exp (-\mathrm{i} 2 k D)}{1-r_{1} r_{2} \exp (-\mathrm{i} 2 k D)}\right),
$$

while the transmitted field becomes

$$
a_{2}=a_{0} \frac{-t_{1} t_{2} \exp (-\mathrm{i} k D)}{1-r_{1} r_{2} \exp (-\mathrm{i} 2 k D)}
$$

The properties of two mirror cavities will be discussed in more detail in Section 5.1.

\subsection{Coupling matrices}

Computations that involve sets of linear equations as shown in Section 2.2 can often be done or written efficiently with matrices. Two methods of applying matrices to coupling field amplitudes are demonstrated below, using again the example of a two mirror cavity. First of all, we can rewrite the coupling equations in matrix form. The mirror coupling as given in Figure 3 becomes

$$
\begin{aligned}
& \text { mirror }(r, t) \\
& \left(\begin{array}{l}
a_{2} \\
a_{4}
\end{array}\right)=\left(\begin{array}{cc}
\mathrm{i} t & r \\
r & \mathrm{i} t
\end{array}\right)\left(\begin{array}{l}
a_{1} \\
a_{3}
\end{array}\right)
\end{aligned}
$$

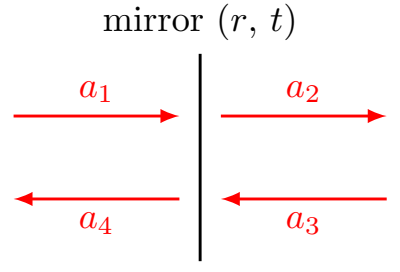

Figure 6 
and the amplitude coupling at a 'space', as given in Figure 4, can be written as

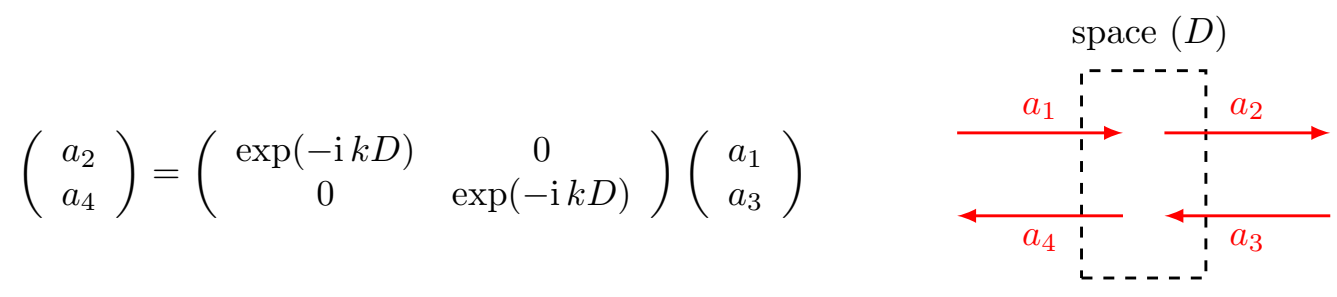

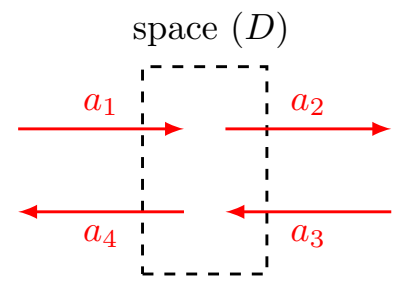

Figure 7

In these examples the matrix simply transforms the 'known' impinging amplitudes into the 'unknown' outgoing amplitudes.

\section{Coupling matrices for numerical computations}

An obvious application of the matrices introduced above would be to construct a large matrix for an extended optical system appropriate for computerisation. A very flexible method is to setup one equation for each field amplitude. The set of linear equations for a mirror would expand to

$$
\left(\begin{array}{cccc}
1 & 0 & 0 & 0 \\
-\mathrm{i} t & 1 & 0 & -r \\
0 & 0 & 1 & 0 \\
-r & 0 & -\mathrm{i} t & 1
\end{array}\right)\left(\begin{array}{c}
a_{1} \\
a_{2} \\
a_{3} \\
a_{4}
\end{array}\right)=\left(\begin{array}{c}
a_{1} \\
0 \\
a_{3} \\
0
\end{array}\right)=M_{\text {system }} \vec{a}_{\text {sol }}=\vec{a}_{\text {input }}
$$

where the input vector ${ }^{1} \vec{a}_{\text {input }}$ has non-zero values for the impinging fields and $\vec{a}_{\text {sol }}$ is the 'solution' vector, i.e., after solving the system of equations the amplitudes of the impinging as well as those of the outgoing fields are stored in that vector.

As an example we apply this method to the two mirror cavity. The system matrix for the optical setup shown in Figure 5 becomes

$$
\left(\begin{array}{ccccccc}
1 & 0 & 0 & 0 & 0 & 0 & 0 \\
-\mathrm{i} t_{1} & 1 & 0 & -r_{1} & 0 & 0 & 0 \\
-r_{1} & 0 & 1 & -\mathrm{i} t_{1} & 0 & 0 & 0 \\
0 & 0 & 0 & 1 & 0 & 0 & -e^{-\mathrm{i} k D} \\
0 & -e^{-\mathrm{i} k D} & 0 & 0 & 1 & 0 & 0 \\
0 & 0 & 0 & 0 & -\mathrm{i} t_{2} & 1 & 0 \\
0 & 0 & 0 & 0 & 0 & -r_{2} & 1
\end{array}\right)\left(\begin{array}{c}
a_{0} \\
a_{1} \\
a_{4} \\
a_{3}^{\prime} \\
a_{1}^{\prime} \\
a_{2} \\
a_{3}
\end{array}\right)=\left(\begin{array}{c}
a_{0} \\
0 \\
0 \\
0 \\
0 \\
0 \\
0
\end{array}\right)
$$

This is a sparse matrix. Sparse matrices are an important subclass of linear algebra problems and many efficient numerical algorithms for solving sparse matrices are freely available (see, for example, [13]). The advantage of this method of constructing a single matrix for an entire optical system is the direct access to all field amplitudes. It also stores each coupling coefficient in one or more dedicated matrix elements, so that numerical values for each parameter can be read out or changed after the matrix has been constructed and, for example, stored in computer memory. The obvious disadvantage is that the size of the matrix quickly grows with the number of optical elements (and with the degrees of freedom of the system, see, for example, Section 7).

\footnotetext{
${ }^{1}$ In many implementations of numerical matrix solvers the input vector is also called the right-hand side vector.
} 


\section{Coupling matrices for a compact system descriptions}

The following method is probably most useful for analytic computations, or for optimisation aspects of a numerical computation. The idea behind the scheme, which is used for computing the characteristics of dielectric coatings $[28,40]$ and has been demonstrated for analysing gravitational wave detectors [43], is to rearrange equations as in Figure 6 and Figure 7 such that the overall matrix describing a series of components can be obtained by multiplication of the component matrices. In order to achieve this, the coupling equations have to be re-ordered so that the input vector consists of two field amplitudes at one side of the component. For the mirror, this gives a coupling matrix of

$$
\left(\begin{array}{l}
a_{1} \\
a_{4}
\end{array}\right)=\frac{\mathrm{i}}{t}\left(\begin{array}{ll}
-1 & r \\
-r & r^{2}+t^{2}
\end{array}\right)\left(\begin{array}{l}
a_{2} \\
a_{3}
\end{array}\right) .
$$

In the special case of the lossless mirror this matrix simplifies as we have $r^{2}+t^{2}=R+T=1$. The space component would be described by the following matrix:

$$
\left(\begin{array}{l}
a_{1} \\
a_{4}
\end{array}\right)=\left(\begin{array}{cc}
\exp (\mathrm{i} k D) & 0 \\
0 & \exp (-\mathrm{i} k D)
\end{array}\right)\left(\begin{array}{l}
a_{2} \\
a_{3}
\end{array}\right) .
$$

With these matrices we can very easily compute a matrix for the cavity with two lossless mirrors as

$$
\begin{aligned}
M_{\text {cav }} & =M_{\text {mirror } 1} \times M_{\text {space }} \times M_{\text {mirror } 2} \\
& =\frac{-1}{t_{1} t_{2}}\left(\begin{array}{cc}
e^{+}-r_{1} r_{2} e^{-} & -r_{2} e^{+}+r_{1} e^{-} \\
-r_{2} e^{-}+r_{1} e^{+} & e^{-}-r_{1} r_{2} e^{+}
\end{array}\right),
\end{aligned}
$$

with $e^{+}=\exp (\mathrm{i} k D)$ and $e^{-}=\exp (-\mathrm{i} k D)$. The system of equation describing a cavity shown in Equation (4) can now be written more compactly as

$$
\left(\begin{array}{l}
a_{0} \\
a_{4}
\end{array}\right)=\frac{-1}{t_{1} t_{2}}\left(\begin{array}{c}
e^{+}-r_{1} r_{2} e^{-}-r_{2} e^{+}+r_{1} e^{-} \\
-r_{2} e^{-}+r_{1} e^{+} e^{-}-r_{1} r_{2} e^{+}
\end{array}\right)\left(\begin{array}{c}
a_{2} \\
0
\end{array}\right) .
$$

This allows direct computation of the amplitude of the transmitted field resulting in

$$
a_{2}=a_{0} \frac{-t_{1} t_{2} \exp (-\mathrm{i} k D)}{1-r_{1} r_{2} \exp (-\mathrm{i} 2 k D)},
$$

which is the same as Equation (8).

The advantage of this matrix method is that it allows compact storage of any series of mirrors and propagations, and potentially other optical elements, in a single $2 \times 2$ matrix. The disadvantage inherent in this scheme is the lack of information about the field amplitudes inside the group of optical elements.

\subsection{Phase relation at a mirror or beam splitter}

The magnitude and phase of reflection at a single optical surface can be derived from Maxwell's equations and the electromagnetic boundary conditions at the surface, and in particular the condition that the field amplitudes tangential to the optical surface must be continuous. The results are called Fresnel's equations [33]. Thus, for a field impinging on an optical surface under normal incidence we can give the reflection coefficient as

$$
r=\frac{n_{1}-n_{2}}{n_{1}+n_{2}},
$$


with $n_{1}$ and $n_{2}$ the indices of refraction of the first and second medium, respectively. The transmission coefficient for a lossless surface can be computed as $t^{2}=1-r^{2}$. We note that the phase change upon reflection is either 0 or $180^{\circ}$, depending on whether the second medium is optically thinner or thicker than the first. It is not shown here but Fresnel's equations can also be used to show that the phase change for the transmitted light at a lossless surface is zero. This contrasts with the definitions given in Section 2.1 (see Figure (3)ff.), where the phase shift upon any reflection is defined as zero and the transmitted light experiences a phase shift of $\pi / 2$. The following section explains the motivation for the latter definition having been adopted as the common notation for the analysis of modern optical systems.

\subsubsection{Composite optical surfaces}

Modern mirrors and beam splitters that make use of dielectric coatings are complex optical systems, see Figure 8 whose reflectivity and transmission depend on the multiple interference inside the coating layers and thus on microscopic parameters. The phase change upon transmission or reflection depends on the details of the applied coating and is typically not known. In any case, the knowledge of an absolute value of a phase change is typically not of interest in laser interferometers because the absolute positions of the optical components are not known to sub-wavelength precision. Instead the relative phase between the incoming and outgoing beams is of importance. In the following we demonstrate how constraints on these relative phases, i.e., the phase relation between the beams, can be derived from the fundamental principle of power conservation. To do this we consider a Michelson interferometer, as shown in Figure 9, with perfectly-reflecting mirrors. The beam splitter of the Michelson interferometer is the object under test. We assume that the magnitude of the reflection $r$ and transmission $t$ are known. The phase changes upon transmission and reflection are unknown. Due to symmetry we can say that the phase change upon transmission $\varphi_{t}$ should be the same in both directions. However, the phase change on reflection might be different for either direction, thus, we write $\varphi_{r 1}$ for the reflection at the front and $\varphi_{r 2}$ for the reflection at the back of the beam splitter.

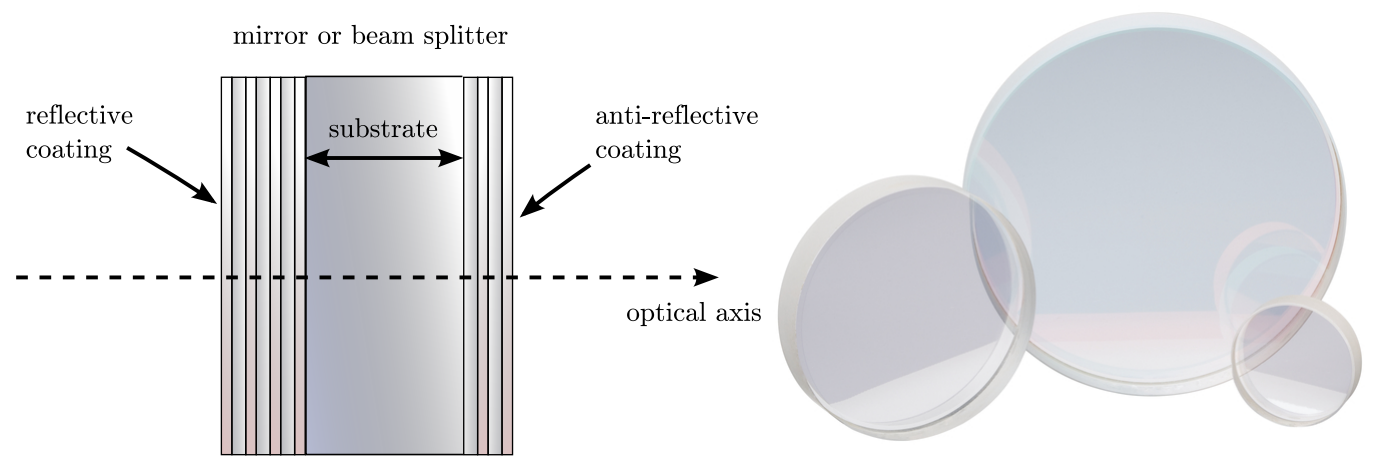

Figure 8: This sketch shows a mirror or beam splitter component with dielectric coatings and the photograph shows some typical commercially available examples [45]. Most mirrors and beam splitters used in optical experiments are of this type: a substrate made from glass, quartz or fused silica is coated on both sides. The reflective coating defines the overall reflectivity of the component (anything between $R \approx 1$ and $R \approx 0$, while the anti-reflective coating is used to reduce the reflection at the second optical surface as much as possible so that this surface does not influence the light. Please note that the drawing is not to scale, the coatings are typically only a few microns thick on a several millimetre to centimetre thick substrate. 


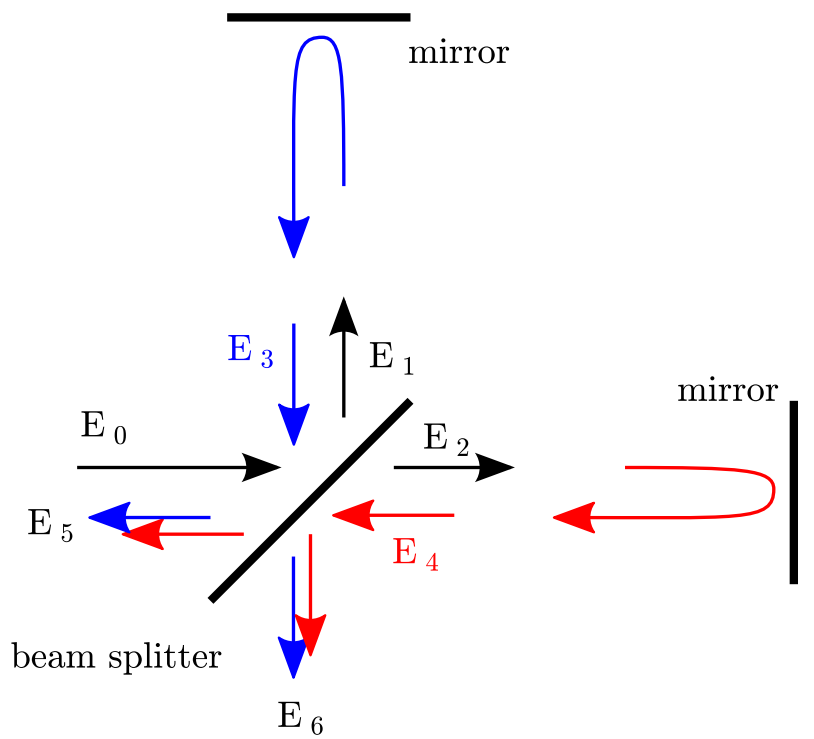

Figure 9: The relation between the phase of the light field amplitudes at a beam splitter can be computed assuming a Michelson interferometer, with arbitrary arm length but perfectly-reflecting mirrors. The incoming field $E_{0}$ is split into two fields $E_{1}$ and $E_{2}$ which are reflected atthe end mirrors and return to the beam splitter, as $E_{3}$ and $E_{4}$, to be recombined into two outgoing fields. These outgoing fields $E_{5}$ and $E_{6}$ are depicted by two arrows to highlight that these are the sum of the transmitted and reflected components of the returning fields. We can derive constraints for the phase of $E_{1}$ and $E_{2}$ with respect to the input field $E_{0}$ from the conservation of energy: $\left|E_{0}\right|^{2}=\left|E_{5}\right|^{2}+\left|E_{6}\right|^{2}$.

Then the electric fields can be computed as

$$
E_{1}=r E_{0} e^{\mathrm{i} \varphi_{r 1}} ; \quad E_{2}=t E_{0} e^{\mathrm{i} \varphi_{t}}
$$

We do not know the length of the interferometer arms. Thus, we introduce two further unknown phases: $\Phi_{1}$ for the total phase accumulated by the field in the vertical arm and $\Phi_{2}$ for the total phase accumulated in the horizontal arm. The fields impinging on the beam splitter compute as

$$
E_{3}=r E_{0} e^{\mathrm{i}\left(\varphi_{r 1}+\Phi_{1}\right)} ; \quad E_{4}=t E_{0} e^{\mathrm{i}\left(\varphi_{t}+\Phi_{2}\right)} .
$$

The outgoing fields are computed as the sums of the reflected and transmitted components:

$$
\begin{aligned}
& E_{5}=E_{0}\left(R e^{\mathrm{i}\left(2 \varphi_{r 1}+\Phi_{1}\right)}+T e^{\mathrm{i}\left(2 \varphi_{t}+\Phi_{2}\right)}\right) \\
& E_{6}=E_{0} r t\left(e^{\mathrm{i}\left(\varphi_{t}+\varphi_{r 1}+\Phi_{1}\right)}+e^{\mathrm{i}\left(\varphi_{t}+\varphi_{r 2}+\Phi_{2}\right)}\right),
\end{aligned}
$$

with $R=r^{2}$ and $T=t^{2}$.

It will be convenient to separate the phase factors into common and differential ones. We can write

$$
E_{5}=E_{0} e^{\mathrm{i} \alpha_{+}}\left(R e^{\mathrm{i} \alpha_{-}}+T e^{-\mathrm{i} \alpha_{-}}\right)
$$

with

$$
\alpha_{+}=\varphi_{r 1}+\varphi_{t}+\frac{1}{2}\left(\Phi_{1}+\Phi_{2}\right) ; \quad \alpha_{-}=\varphi_{r 1}-\varphi_{t}+\frac{1}{2}\left(\Phi_{1}-\Phi_{2}\right),
$$

and similarly

$$
E_{6}=E_{0} r t e^{\mathrm{i} \beta_{+}} 2 \cos \left(\beta_{-}\right),
$$


with

$$
\beta_{+}=\varphi_{t}+\frac{1}{2}\left(\varphi_{r 1}+\varphi_{r 2}+\Phi_{1}+\Phi_{2}\right) ; \quad \beta_{-}=\frac{1}{2}\left(\varphi_{r 1}-\varphi_{r 2}+\Phi_{1}-\Phi_{2}\right) .
$$

For simplicity we now limit the discussion to a 50:50 beam splitter with $r=t=1 / \sqrt{2}$, for which we can simplify the field expressions even further:

$$
E_{5}=E_{0} e^{\mathrm{i} \alpha_{+}} \cos \left(\alpha_{-}\right) ; \quad E_{6}=E_{0} e^{\mathrm{i} \beta_{+}} \cos \left(\beta_{-}\right) .
$$

Conservation of energy requires that $\left|E_{0}\right|^{2}=\left|E_{5}\right|^{2}+\left|E_{6}\right|^{2}$, which in turn requires

$$
\cos ^{2}\left(\alpha_{-}\right)+\cos ^{2}\left(\beta_{-}\right)=1
$$

which is only true if

$$
\alpha_{-}-\beta_{-}=(2 N+1) \frac{\pi}{2}
$$

with $N$ as in integer (positive, negative or zero). This gives the following constraint on the phase factors

$$
\frac{1}{2}\left(\varphi_{r 1}+\varphi_{r 2}\right)-\varphi_{t}=(2 N+1) \frac{\pi}{2}
$$

One can show that exactly the same condition results in the case of arbitrary (lossless) reflectivity of the beam splitter [48].

We can test whether two known examples fulfill this condition. If the beam-splitting surface is the front of a glass plate we know that $\varphi_{t}=0, \varphi_{r 1}=\pi, \varphi_{r 2}=0$, which conforms with Equation (28). A second example is the two-mirror resonator, see Section 2.2. If we consider the cavity as an optical 'black box', it also splits any incoming beam into a reflected and transmitted component, like a mirror or beam splitter. Further we know that a symmetric resonator must give the same results for fields injected from the left or from the right. Thus, the phase factors upon reflection must be equal $\varphi_{r}=\varphi_{r 1}=\varphi_{r 2}$. The reflection and transmission coefficients are given by Equations (7) and (8) as

and

$$
r_{\mathrm{cav}}=\left(r_{1}-\frac{r_{2} t_{1}^{2} \exp (-\mathrm{i} 2 k D)}{1-r_{1} r_{2} \exp (-\mathrm{i} 2 k D)}\right)
$$

$$
t_{\mathrm{cav}}=\frac{-t_{1} t_{2} \exp (-\mathrm{i} k D)}{1-r_{1} r_{2} \exp (-\mathrm{i} 2 k D)}
$$

We demonstrate a simple case by putting the cavity on resonance $(k D=N \pi)$. This yields

$$
r_{\mathrm{cav}}=\left(r_{1}-\frac{r_{2} t_{1}^{2}}{1-r_{1} r_{2}}\right) ; \quad t_{\mathrm{cav}}=\frac{\mathrm{i} t_{1} t_{2}}{1-r_{1} r_{2}},
$$

with $r_{\text {cav }}$ being purely real and $t_{\text {cav }}$ imaginary and thus $\varphi_{t}=\pi / 2$ and $\varphi_{r}=0$ which also agrees with Equation (28).

In most cases we neither know nor care about the exact phase factors. Instead we can pick any set which fulfills Equation (28). For this document we have chosen to use phase factors equal to those of the cavity, i.e., $\varphi_{t}=\pi / 2$ and $\varphi_{r}=0$, which is why we write the reflection and transmission at a mirror or beam splitter as

$$
E_{\mathrm{refl}}=r E_{0} \quad \text { and } \quad E_{\mathrm{trans}}=\mathrm{i} t E_{0} .
$$

In this definition $r$ and $t$ are positive real numbers satisfying $r^{2}+t^{2}=1$ for the lossless case.

Please note that we only have the freedom to chose convenient phase factors when we do not know or do not care about the details of the optical system, which performs the beam splitting. If instead the details are important, for example when computing the properties of a thin coating layer, such as anti-reflex coatings, the proper phase factors for the respective interfaces must be computed and used. 


\subsection{Lengths and tunings: numerical accuracy of distances}

The resonance condition inside an optical cavity and the operating point of an interferometer depends on the optical path lengths modulo the laser wavelength, i.e., for light from an Nd:YAG laser length differences of less than $1 \mu \mathrm{m}$ are of interest, not the full magnitude of the distances between optics. On the other hand, several parameters describing the general properties of an optical system, like the finesse or free spectral range of a cavity (see Section 5.1) depend on the macroscopic distance and do not change significantly when the distance is changed on the order of a wavelength. This illustrates that the distance between optical components might not be the best parameter to use for the analysis of optical systems. Furthermore, it turns out that in numerical algorithms the distance may suffer from rounding errors. Let us use the Virgo [56] arm cavities as an example to illustrate this. The cavity length is approximately $3 \mathrm{~km}$, the wavelength is on the order of $1 \mathrm{\mu m}$, the mirror positions are actively controlled with a precision of $1 \mathrm{pm}$ and the detector sensitivity can be as good as $10^{-18} \mathrm{~m}$, measured on $\sim 10 \mathrm{~ms}$ timescales (i.e., many samples of the data acquisition rate). The floating point accuracy of common, fast numerical algorithms is typically not better than $10^{-15}$. If we were to store the distance between the cavity mirrors as such a floating point number, the accuracy would be limited to $3 \mathrm{pm}$, which does not even cover the accuracy of the control systems, let alone the sensitivity.

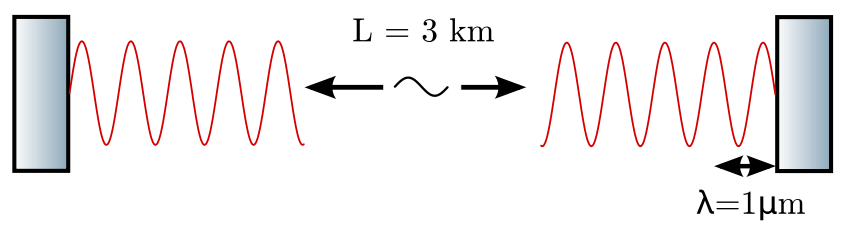

Figure 10: Illustration of an arm cavity of the Virgo gravitational-wave detector [56]: the macroscopic length $L$ of the cavity is approximately $3 \mathrm{~km}$, while the wavelength of the Nd:YAG laser is $\lambda \approx 1 \mu \mathrm{m}$. The resonance condition is only affected by the microscopic position of the wave nodes with respect to the mirror surfaces and not by the macroscopic length, i.e., displacement of one mirror by $\Delta x=\lambda / 2$ re-creates exactly the same condition. However, other parameters of the cavity, such as the finesse, only depend on the macroscopic length $L$ and not on the microscopic tuning.

A simple and elegant solution to this problem is to split a distance $D$ between two optical components into two parameters [29]: one is the macroscopic 'length' $L$, defined as the multiple of a constant wavelength $\lambda_{0}$ yielding the smallest difference to $D$. The second parameter is the microscopic tuning $T$ that is defined as the remaining difference between $L$ and $D$, i.e., $D=L+T$. Typically, $\lambda_{0}$ can be understood as the wavelength of the laser in vacuum, however, if the laser frequency changes during the experiment or multiple light fields with different frequencies are used simultaneously, a default constant wavelength must be chosen arbitrarily. Please note that usually the term $\lambda$ in any equation refers to the actual wavelength at the respective location as $\lambda=\lambda_{0} / n$ with $n$ the index of refraction at the local medium.

We have seen in Section 2.1 that distances appear in the expressions for electromagnetic waves in connection with the wave number, for example,

$$
E_{2}=E_{1} \exp (-\mathrm{i} k z)
$$

Thus, the difference in phase between the field at $z=z_{1}$ and $z=z_{1}+D$ is given as

$$
\varphi=-k D .
$$

We recall that $k=2 \pi / \lambda=\omega / c$. We can define $\omega_{0}=2 \pi c / \lambda_{0}$ and $k_{0}=\omega_{0} / c$. For any given wavelength $\lambda$ we can write the corresponding frequency as a sum of the default frequency and a 
difference frequency $\omega=\omega_{0}+\Delta \omega$. Using these definitions, we can rewrite Equation (34) with length and tuning as

$$
-\varphi=k D=\frac{\omega_{0} L}{c}+\frac{\Delta \omega L}{c}+\frac{\omega_{0} T}{c}+\frac{\Delta \omega T}{c} .
$$

The first term of the sum is always a multiple of $2 \pi$, which is equivalent to zero. The last term of the sum is the smallest, approximately of the order $\Delta \omega \cdot 10^{-14}$. For typical values of $L \approx 1 \mathrm{~m}$, $T<1 \mu \mathrm{m}$ and $\Delta \omega<2 \pi \cdot 100 \mathrm{MHz}$ we find that

$$
\frac{\omega_{0} L}{c}=0, \quad \frac{\Delta \omega L}{c} \lesssim 2, \quad \frac{\omega_{0} T}{c} \lesssim 6, \quad \frac{\Delta \omega T}{c} \lesssim 210^{-6}
$$

which shows that the last term can often be ignored.

We can also write the tuning directly as a phase. We define as the dimensionless tuning

$$
\phi=\omega_{0} T / c .
$$

This yields

$$
\exp \left(\mathrm{i} \frac{\omega}{c} T\right)=\exp \left(\mathrm{i} \frac{\omega_{0}}{c} T \frac{\omega}{\omega_{0}}\right)=\exp \left(\mathrm{i} \frac{\omega}{\omega_{0}} \phi\right) .
$$

The tuning $\phi$ is given in radian with $2 \pi$ referring to a microscopic distance of one wavelength ${ }^{2} \lambda_{0}$.

Finally, we can write the following expression for the phase difference between the light field taken at the end points of a distance $D$ :

$$
\varphi=-k D=-\left(\frac{\Delta \omega L}{c}+\phi \frac{\omega}{\omega_{0}}\right)
$$

or if we neglect the last term from Equation $(36)$ we can approximate $\left(\omega / \omega_{0} \approx 1\right)$ to obtain

$$
\varphi \approx-\left(\frac{\Delta \omega L}{c}+\phi\right) .
$$

This convention provides two parameters $L$ and $\phi$, that can describe distances with a markedly improved numerical accuracy. In addition, this definition often allows simplification of the algebraic notation of interferometer signals. By convention we associate a length $L$ with the propagation through free space, whereas the tuning will be treated as a parameter of the optical components. Effectively the tuning then represents a microscopic displacement of the respective component. If, for example, a cavity is to be resonant to the laser light, the tunings of the mirrors have to be the same whereas the length of the space in between can be arbitrary.

\footnotetext{
${ }^{2}$ Note that in other publications the tuning or equivalent microscopic displacements are sometimes defined via an optical path-length difference. In that case, a tuning of $2 \pi$ is used to refer to the change of the optical path length of one wavelength, which, for example, if the reflection at a mirror is described, corresponds to a change of the mirror's position of $\lambda_{0} / 2$.
} 


\subsection{Revised coupling matrices for space and mirrors}

Using the definitions for length and tunings we can rewrite the coupling equations for mirrors and spaces introduced in Section 2.1 as follows. The mirror coupling becomes

$$
\begin{aligned}
& \text { mirror }(r, t, \phi) \\
& \left(\begin{array}{c}
a_{2} \\
a_{4}
\end{array}\right)=\left(\begin{array}{cc}
\mathrm{i} t & r \exp \left(\mathrm{i} 2 \phi \frac{\omega}{\omega_{0}}\right) \\
r \exp \left(-\mathrm{i} 2 \phi \frac{\omega}{\omega_{0}}\right) & \mathrm{i} t
\end{array}\right)\left(\begin{array}{l}
a_{1} \\
a_{3}
\end{array}\right)
\end{aligned}
$$

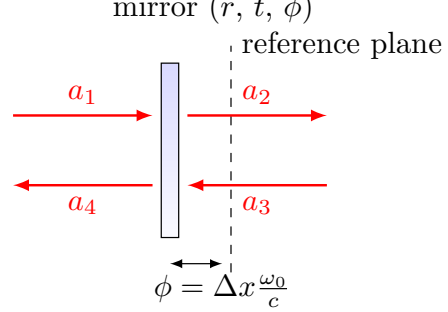

Figure 11

(compare this to Figure 6), and the amplitude coupling for a 'space', formally written as in Figure 7, is now written as

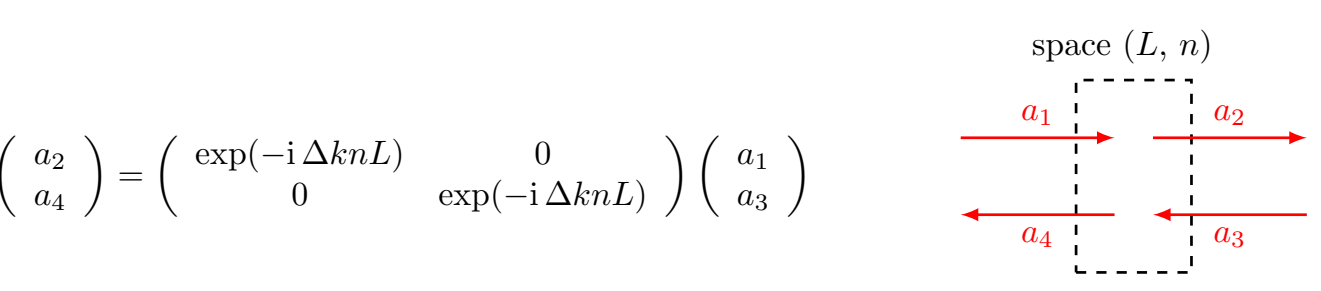

Figure 12 


\subsection{Finesse examples}

\subsubsection{Mirror reflectivity and transmittance}
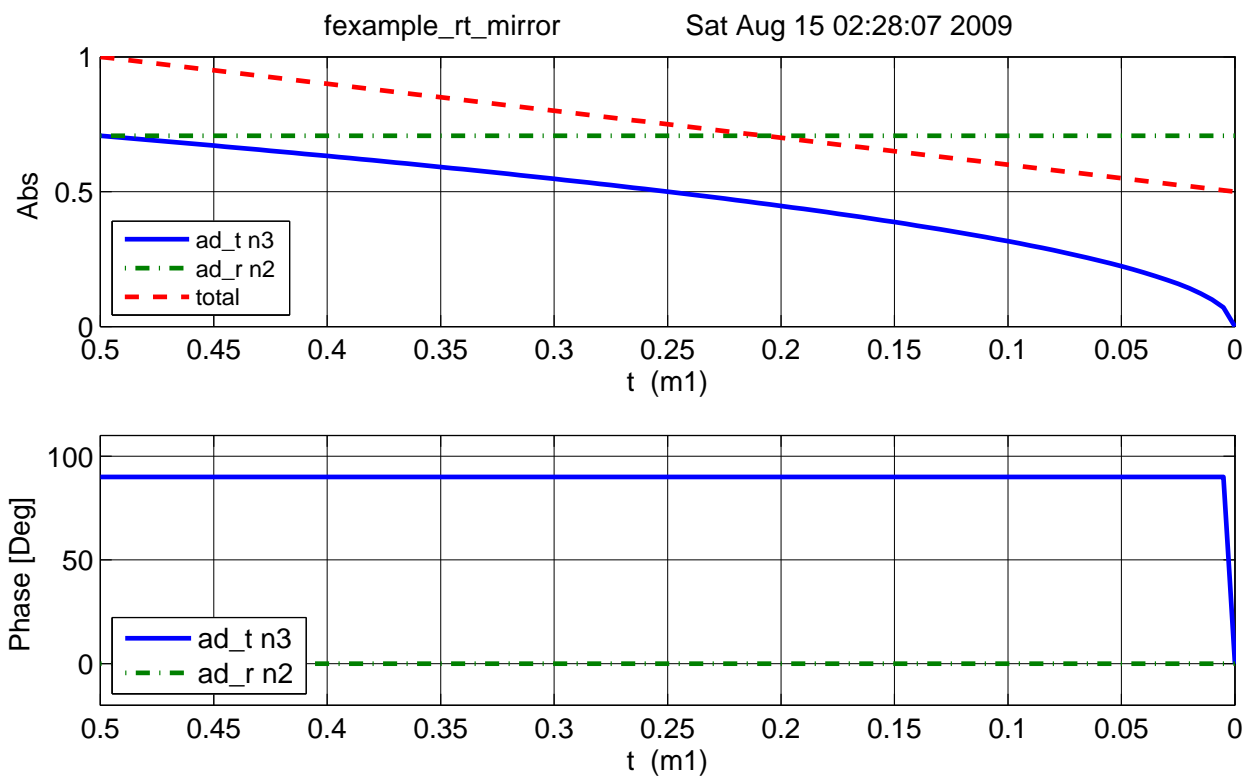

Figure 13: FINESSE example: Mirror reflectivity and transmittance.

We use Finesse to plot the amplitudes of the light fields transmitted and reflected by a mirror (given by a single surface). Initially, the mirror has a power reflectance and transmittance of $R=T=0.5$ and is, thus, lossless. For the plot in Figure 13 we tune the transmittance from 0.5 to 0 . Since we do not explicitly change the reflectivity, $R$ remains at 0.5 and the mirror loss increases instead, which is shown by the trace labelled 'total' corresponding to the sum of the reflected and transmitted light power. The plot also shows the phase convention of a $90^{\circ}$ phase shift for the transmitted light.

\section{Finesse input file for 'Mirror reflectivity and transmittance'}

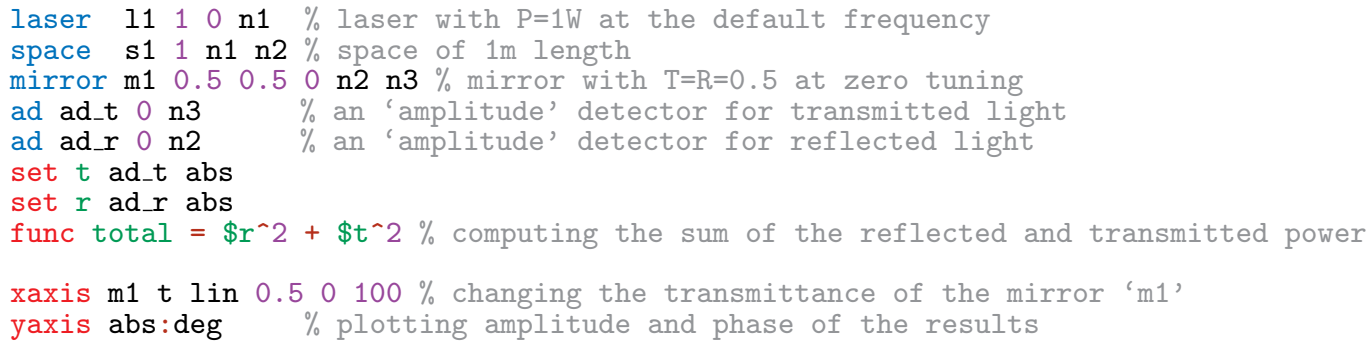

\subsubsection{Length and tunings}

This Finesse file demonstrates the conventions for lengths and microscopic positions introduced in Section 2.5. The top trace in Figure 14 depicts the phase change of a beam reflected by a beam splitter as the function of the beam splitter tuning. By changing the tuning from 0 to $180^{\circ}$ 

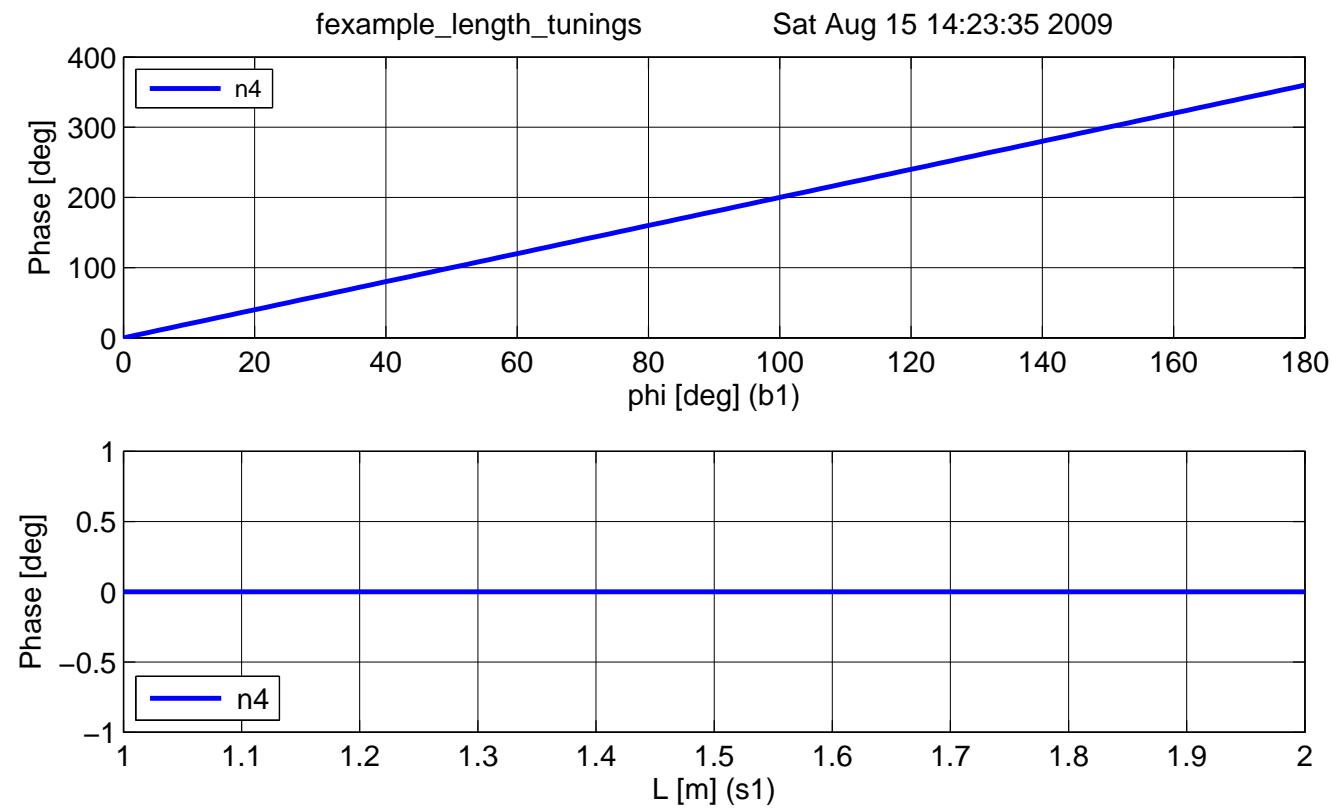

Figure 14: FinESSE example: Length and tunings.

the beam splitter is moved forward and shortens the path length by one wavelength, which by convention increases the light phase by $360^{\circ}$. On the other hand, if a length of a space is changed, the phase of the transmitted light is unchanged (for the default wavelength $\Delta k=0$ ), as shown the in the lower trace.

\section{Finesse input file for 'Length and tunings'}

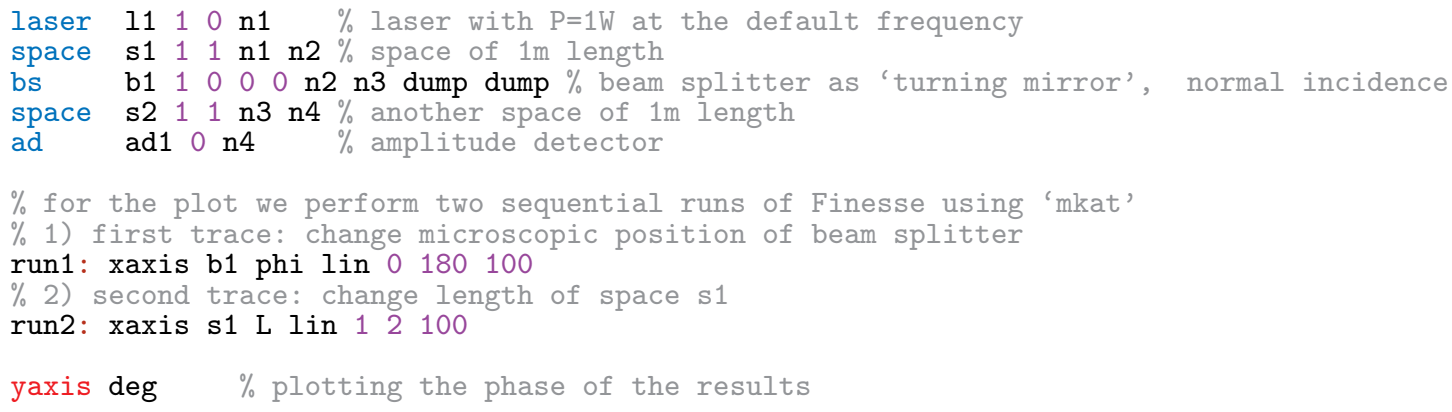




\section{Light with Multiple Frequency Components}

So far we have considered the electromagnetic field to be monochromatic. This has allowed us to compute light-field amplitudes in a quasi-static optical setup. In this section, we introduce the frequency of the light as a new degree of freedom. In fact, we consider a field consisting of a finite and discrete number of frequency components. We write this as

$$
E(t, z)=\sum_{j} a_{j} \exp \left(\mathrm{i}\left(\omega_{j} t-k_{j} z\right)\right)
$$

with complex amplitude factors $a_{j}, \omega_{j}$ as the angular frequency of the light field and $k_{j}=\omega_{j} / c$. In many cases the analysis compares different fields at one specific location only, in which case we can set $z=0$ and write

$$
E(t)=\sum_{j} a_{j} \exp \left(\mathrm{i} \omega_{j} t\right)
$$

In the following sections the concept of light modulation is introduced. As this inherently involves light fields with multiple frequency components, it makes use of this type of field description. Again we start with the two-mirror cavity to illustrate how the concept of modulation can be used to model the effect of mirror motion.

a)

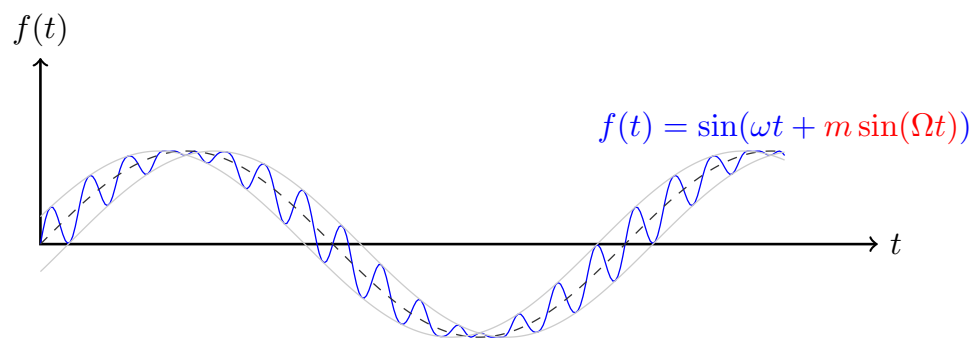

b)

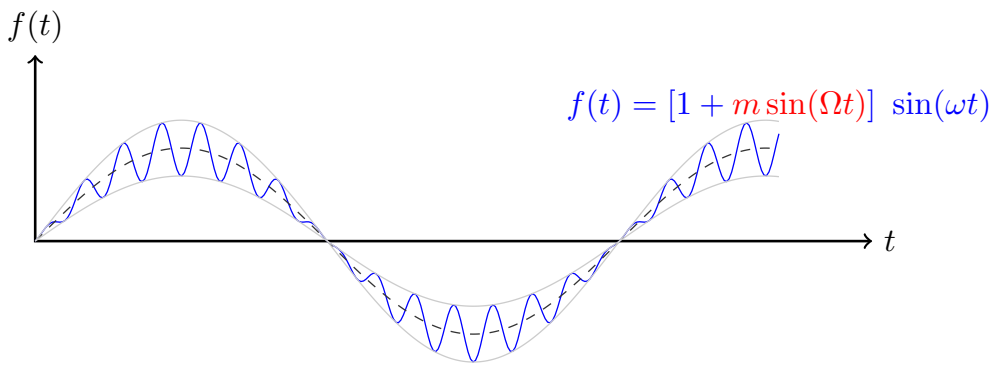

Figure 15: Example traces for phase and amplitude modulation: the upper plot a) shows a phasemodulated sine wave and the lower plot b) depicts an amplitude-modulated sine wave. Phase modulation is characterised by the fact that it mostly affects the zero crossings of the sine wave. Amplitude modulation affects mostly the maximum amplitude of the wave. The equations show the modulation terms in red with $m$ the modulation index and $\Omega$ the modulation frequency.

\subsection{Modulation of light fields}

Laser interferometers typically use three different types of light fields: the laser with a frequency of, for example, $f \approx 2.8 \cdot 10^{14} \mathrm{~Hz}$, radio frequency $(\mathrm{RF})$ sidebands used for interferometer control with frequencies (offset to the laser frequency) of $f \approx 1 \cdot 10^{6}$ to $150 \cdot 10^{6} \mathrm{~Hz}$, and the signal sidebands at frequencies of 1 to $10,000 \mathrm{~Hz}^{3}$. As these modulations usually have as their origin a change in

\footnotetext{
${ }^{3}$ The signal sidebands are sometimes also called audio sidebands because of their frequency range.
} 
optical path length, they are often phase modulations of the laser frequency, the RF sidebands are utilised for optical readout purposes, while the signal sidebands carry the signal to be measured (the gravitational-wave signal plus noise created in the interferometer).

Figure 15 shows a time domain representation of an electromagnetic wave of frequency $\omega_{0}$, whose amplitude or phase is modulated at a frequency $\Omega$. One can easily see some characteristics of these two types of modulation, for example, that amplitude modulation leaves the zero crossing of the wave unchanged whereas with phase modulation the maximum and minimum amplitude of the wave remains the same. In the frequency domain in which a modulated field is expanded into several unmodulated field components, the interpretation of modulation becomes even easier: any sinusoidal modulation of amplitude or phase generates new field components, which are shifted in frequency with respect to the initial field. Basically, light power is shifted from one frequency component, the carrier, to several others, the sidebands. The relative amplitudes and phases of these sidebands differ for different types of modulation and different modulation strengths. This section demonstrates how to compute the sideband components for amplitude, phase and frequency modulation.

\subsection{Phase modulation}

Phase modulation can create a large number of sidebands. The number of sidebands with noticeable power depends on the modulation strength (or depth) given by the modulation index $m$. Assuming an input field

$$
E_{\text {in }}=E_{0} \exp \left(\mathrm{i} \omega_{0} t\right)
$$

a sinusoidal phase modulation of the field can be described as

$$
E=E_{0} \exp \left(\mathrm{i}\left(\omega_{0} t+m \cos (\Omega t)\right)\right) .
$$

This equation can be expanded using the identity [27]

$$
\exp (\mathrm{i} z \cos \varphi)=\sum_{k=-\infty}^{\infty} \mathrm{i}^{k} J_{k}(z) \exp (\mathrm{i} k \varphi)
$$

with Bessel functions of the first kind $J_{k}(m)$. We can write

$$
E=E_{0} \exp \left(\mathrm{i} \omega_{0} t\right) \sum_{k=-\infty}^{\infty} \mathrm{i}^{k} J_{k}(m) \exp (\mathrm{i} k \Omega t) .
$$

The field for $k=0$, oscillating with the frequency of the input field $\omega_{0}$, represents the carrier. The sidebands can be divided into upper $(k>0)$ and lower $(k<0)$ sidebands. These sidebands are light fields that have been shifted in frequency by $k \Omega$. The upper and lower sidebands with the same absolute value of $k$ are called a pair of sidebands of order $k$. Equation (46) shows that the carrier is surrounded by an infinite number of sidebands. However, for small modulation indices $(m<1)$ the Bessel functions rapidly decrease with increasing $k$ (the lowest orders of the Bessel functions are shown in Figure 16). For small modulation indices we can use the approximation [2]

$$
J_{k}(m)=\left(\frac{m}{2}\right)^{k} \sum_{n=0}^{\infty} \frac{\left(-\frac{m^{2}}{4}\right)^{n}}{n !(k+n) !}=\frac{1}{k !}\left(\frac{m}{2}\right)^{k}+O\left(m^{k+2}\right) .
$$

In which case, only a few sidebands have to be taken into account. For $m \ll 1$ we can write

$$
\begin{aligned}
E= & E_{0} \exp \left(\mathrm{i} \omega_{0} t\right) \\
& \times\left(J_{0}(m)-\mathrm{i} J_{-1}(m) \exp (-\mathrm{i} \Omega t)+\mathrm{i} J_{1}(m) \exp (\mathrm{i} \Omega t)\right),
\end{aligned}
$$


and with

$$
J_{-k}(m)=(-1)^{k} J_{k}(m),
$$

we obtain

$$
E=E_{0} \exp \left(\mathrm{i} \omega_{0} t\right)\left(1+\mathrm{i} \frac{m}{2}(\exp (-\mathrm{i} \Omega t)+\exp (\mathrm{i} \Omega t))\right),
$$

as the first-order approximation in $m$. In the above equation the carrier field remains unchanged by the modulation, therefore this approximation is not the most intuitive. It is clearer if the approximation up to the second order in $m$ is given:

$$
E=E_{0} \exp \left(\mathrm{i} \omega_{0} t\right)\left(1-\frac{m^{2}}{4}+\mathrm{i} \frac{m}{2}(\exp (-\mathrm{i} \Omega t)+\exp (\mathrm{i} \Omega t))\right)
$$

which shows that power is transferred from the carrier to the sideband fields.

Higher-order expansions in $m$ can be performed simply by specifying the highest order of Bessel function, which is to be used in the sum in Equation (46), i.e.,

$$
E=E_{0} \exp \left(\mathrm{i} \omega_{0} t\right) \sum_{k=- \text { order }}^{\text {order }} i^{k} J_{k}(m) \exp (\mathrm{i} k \Omega t) \text {. }
$$

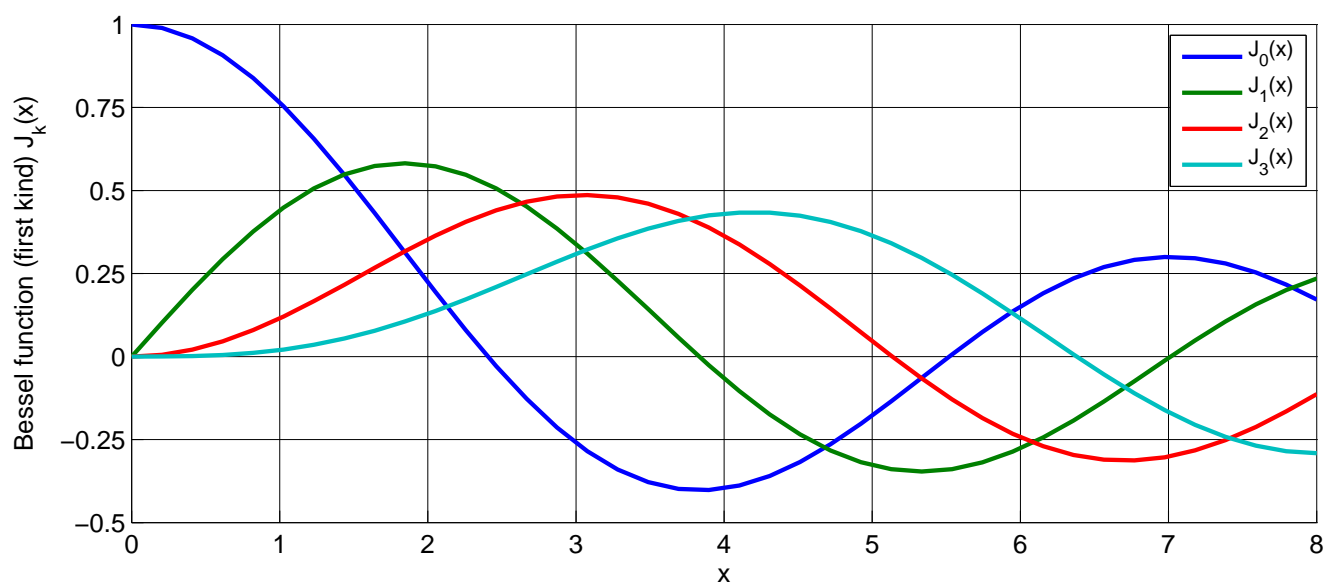

Figure 16: Some of the lowest-order Bessel functions $J_{k}(x)$ of the first kind. For small $x$ the expansion shows a simple $x^{k}$ dependency and higher-order functions can often be neglected.

\subsection{Frequency modulation}

For small modulation, indices, phase modulation and frequency modulation can be understood as different descriptions of the same effect [29]. Following the same spirit as above we would assume a modulated frequency to be given by

$$
\omega=\omega_{0}+m^{\prime} \cos (\Omega t)
$$

and then we might be tempted to write

$$
E=E_{0} \exp \left(\mathrm{i}\left(\omega_{0}+m^{\prime} \cos (\Omega t)\right) t\right)
$$


which would be wrong. The frequency of a wave is actually defined as $\omega /(2 \pi)=f=d \varphi / d t$. Thus, to obtain the frequency given in Equation (53), we need to have a phase of

$$
\omega_{0} t+\frac{m^{\prime}}{\Omega} \sin (\Omega t)
$$

For consistency with the notation for phase modulation, we define the modulation index to be

$$
m=\frac{m^{\prime}}{\Omega}=\frac{\Delta \omega}{\Omega},
$$

with $\Delta \omega$ as the frequency swing - how far the frequency is shifted by the modulation - and $\Omega$ the modulation frequency - how fast the frequency is shifted. Thus, a sinusoidal frequency modulation can be written as

$$
E=E_{0} \exp (\mathrm{i} \varphi)=E_{0} \exp \left(\mathrm{i}\left(\omega_{0} t+\frac{\Delta \omega}{\Omega} \cos (\Omega t)\right)\right),
$$

which is exactly the same expression as Equation (44) for phase modulation. The practical difference is the typical size of the modulation index, with phase modulation having a modulation index of $m<10$, while for frequency modulation, typical numbers might be $m>10^{4}$. Thus, in the case of frequency modulation, the approximations for small $m$ are not valid. The series expansion using Bessel functions, as in Equation (46), can still be performed, however, very many terms of the resulting sum need to be taken into account.

\subsection{Amplitude modulation}

In contrast to phase modulation, (sinusoidal) amplitude modulation always generates exactly two sidebands. Furthermore, a natural maximum modulation index exists: the modulation index is defined to be one $(m=1)$ when the amplitude is modulated between zero and the amplitude of the unmodulated field.

If the amplitude modulation is performed by an active element, for example by modulating the current of a laser diode, the following equation can be used to describe the output field:

$$
\begin{aligned}
E & =E_{0} \exp \left(\mathrm{i} \omega_{0} t\right)(1+m \cos (\Omega t)) \\
& =E_{0} \exp \left(\mathrm{i} \omega_{0} t\right)\left(1+\frac{m}{2} \exp (\mathrm{i} \Omega t)+\frac{m}{2} \exp (-\mathrm{i} \Omega t)\right) .
\end{aligned}
$$

However, passive amplitude modulators (like acousto-optic modulators or electro-optic modulators with polarisers) can only reduce the amplitude. In these cases, the following equation is more useful:

$$
\begin{aligned}
E & =E_{0} \exp \left(\mathrm{i} \omega_{0} t\right)\left(1-\frac{m}{2}(1-\cos (\Omega t))\right) \\
& =E_{0} \exp \left(\mathrm{i} \omega_{0} t\right)\left(1-\frac{m}{2}+\frac{m}{4} \exp (\mathrm{i} \Omega t)+\frac{m}{4} \exp (-\mathrm{i} \Omega t)\right) .
\end{aligned}
$$

\subsection{Sidebands as phasors in a rotating frame}

A common method of visualising the behaviour of sideband fields in interferometers is to use phase diagrams in which each field amplitude is represented by an arrow in the complex plane.

We can think of the electric field amplitude $E_{0} \exp \left(\mathrm{i} \omega_{0} t\right)$ as a vector in the complex plane, rotating around the origin with angular velocity $\omega_{0}$. To illustrate or to help visualise the addition of several light fields it can be useful to look at this problem using a rotating reference frame, 

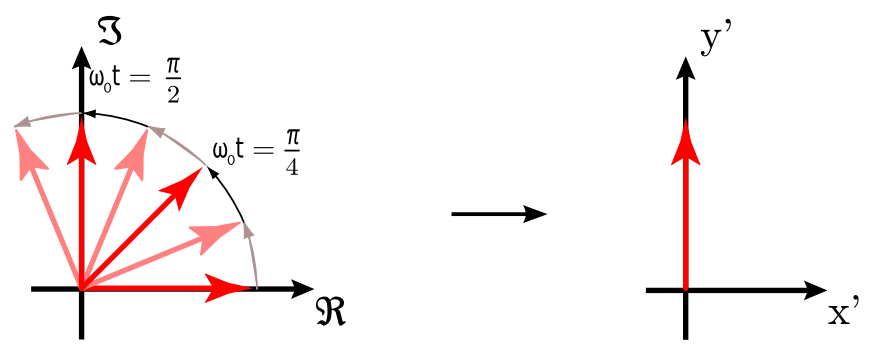

Figure 17: Electric field vector $E_{0} \exp \left(\mathrm{i} \omega_{0} t\right)$ depicted in the complex plane and in a rotating frame $\left(x^{\prime}\right.$, $\left.y^{\prime}\right)$ rotating at $\omega_{0}$ so that the field vector appears stationary.

a)

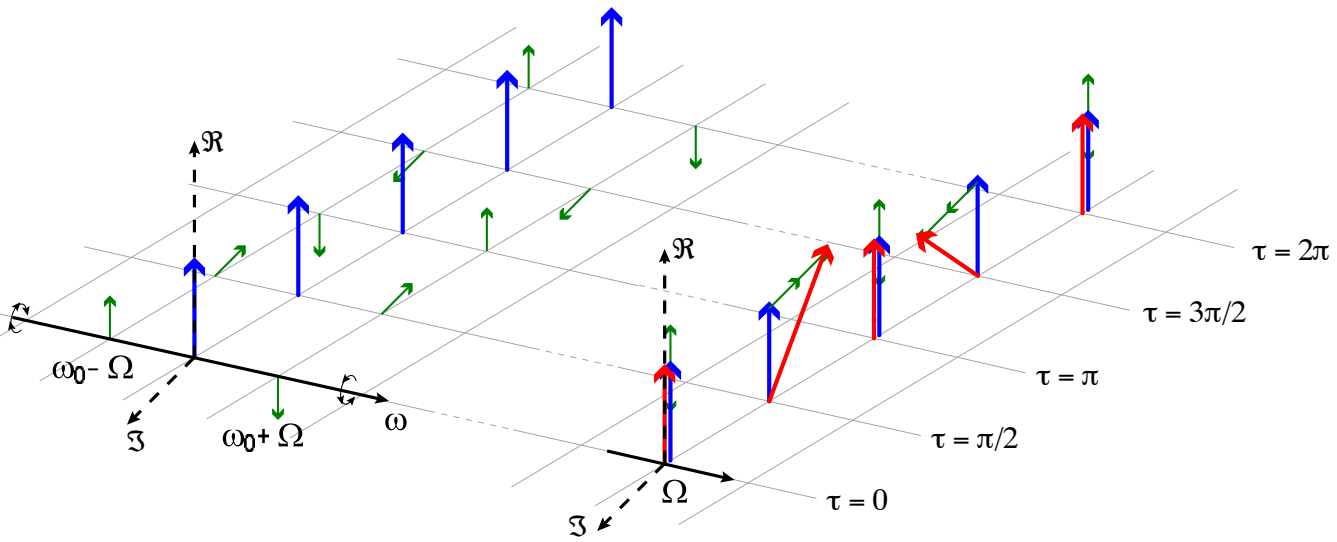

b)

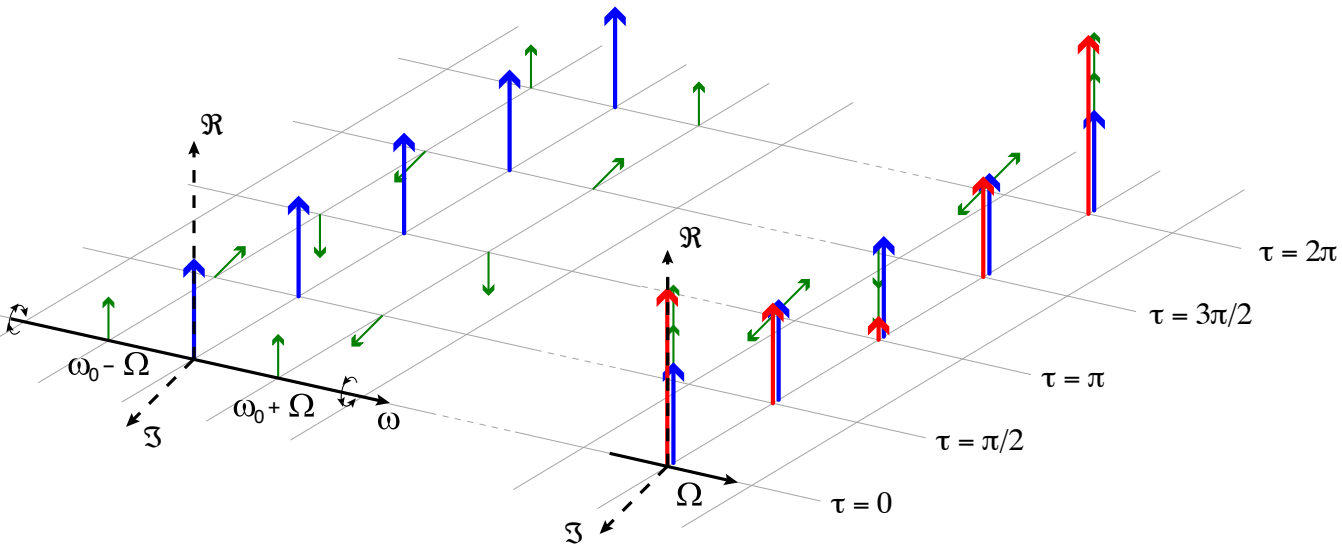

Figure 18: Amplitude and phase modulation in the 'phasor' picture. The upper plots a) illustrate how a phasor diagram can be used to describe phase modulation, while the lower plots b) do the same for amplitude modulation. In both cases the left hand plot shows the carrier in blue and the modulation sidebands in green as snapshots at certain time intervals. One can see clearly that the upper sideband $\left(\omega_{0}+\Omega\right)$ rotates faster than the carrier, while the lower sideband rotates slower. The right plot in both cases shows how the total field vector at any given time can be constructed by adding the three field vectors of the carrier and sidebands. [Drawing courtesy of Simon Chelkowski] 
defined as follows. A complex number shall be defined as $z=x+\mathrm{i} y$ so that the real part is plotted along the $x$-axis, while the $y$-axis is used for the imaginary part. We want to construct a new coordinate system $\left(x^{\prime}, y^{\prime}\right)$ in which the field vector is at a constant position. This can be achieved by defining

or

$$
\begin{aligned}
& x=x^{\prime} \quad \cos \omega_{0} t-y^{\prime} \sin \omega_{0} t \\
& y=x^{\prime} \sin \omega_{0} t+y^{\prime} \cos \omega_{0} t,
\end{aligned}
$$

$$
\begin{aligned}
& x^{\prime}=x \cos \left(-\omega_{0} t\right)-y \sin \left(-\omega_{0} t\right) \\
& y^{\prime}=x \sin \left(-\omega_{0} t\right)+y \cos \left(-\omega_{0} t\right) .
\end{aligned}
$$

Figure 17 illustrates how the transition into the rotating frame makes the field vector to appear stationary. The angle of the field vector in a rotating frame depicts the phase offset of the field. Therefore these vectors are also called phasors and the illustrations using phasors are called phasor diagrams. Two more complex examples of how phasor diagrams can be employed is shown in Figure 18 [11].

Phasor diagrams can be especially useful to see how frequency coupling of light field amplitudes can change the type of modulation, for example, to turn phase modulation into amplitude modulation. An extensive introduction to this type of phasor diagram can be found in [39].

\subsection{Phase modulation through a moving mirror}

Several optical components can modulate transmitted or reflected light fields. In this section we discuss in detail the example of phase modulation by a moving mirror. Mirror motion does not change the transmitted light; however, the phase of the reflected light will be changed as shown in Equation (11).

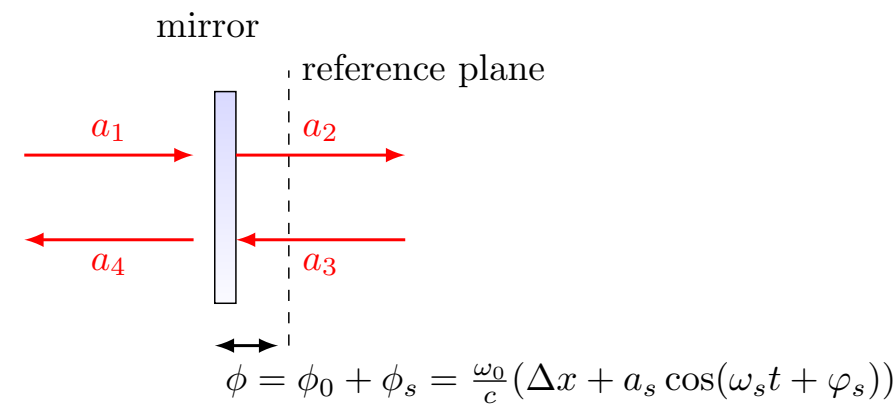

Figure 19: A sinusoidal signal with amplitude $a_{s}$ frequency $\omega_{s}$ and phase offset $\varphi_{s}$ is applied to a mirror position, or to be precise, to the mirror tuning. The equation given for the tuning $\phi$ assumes that $\omega_{s} / \omega_{0} \ll 1$, see Section 2.5 .

We assume sinusoidal change of the mirror's tuning as shown in Figure 19. The position modulation is given as $x_{\mathrm{m}}=a_{\mathrm{s}} \cos \left(\omega_{\mathrm{s}} t+\varphi_{\mathrm{s}}\right)$, and thus the reflected field at the mirror becomes (assuming $a_{4}=0$ )

$$
a_{3}=r a_{1} \exp \left(-\mathrm{i} 2 \phi_{0}\right) \exp \left(\mathrm{i} 2 k x_{\mathrm{m}}\right) \approx r a_{1} \exp \left(-\mathrm{i} 2 \phi_{0}\right) \exp \left(\mathrm{i} 2 k_{0} a_{\mathrm{s}} \cos \left(\omega_{\mathrm{s}} t+\varphi_{\mathrm{s}}\right)\right),
$$

setting $m=2 k_{0} a_{\mathrm{s}}$. This can be expressed as

$$
\begin{aligned}
a_{3}= & r a_{1} \exp \left(-\mathrm{i} 2 \phi_{0}\right)\left(1+\mathrm{i} \frac{m}{2} \exp \left(-\mathrm{i}\left(\omega_{\mathrm{s}} t+\varphi_{\mathrm{s}}\right)\right)+\mathrm{i} \frac{m}{2} \exp \left(\mathrm{i}\left(\omega_{\mathrm{s}} t+\varphi_{\mathrm{s}}\right)\right)\right) \\
= & r a_{1} \exp \left(-\mathrm{i} 2 \phi_{0}\right)\left(1+\frac{m}{2} \exp \left(-\mathrm{i}\left(\omega_{\mathrm{s}} t+\varphi_{\mathrm{s}}-\pi / 2\right)\right)\right. \\
& \left.+\frac{m}{2} \exp \left(\mathrm{i}\left(\omega_{\mathrm{s}} t+\varphi_{\mathrm{s}}+\pi / 2\right)\right)\right) .
\end{aligned}
$$




\subsection{Coupling matrices for beams with multiple frequency components}

The coupling between electromagnetic fields at optical components introduced in Section 2 referred only to the amplitude and phase of a simplified monochromatic field, ignoring all the other parameters of the electric field of the beam given in Equation (1). However, this mathematical concept can be extended to include other parameters provided that we can find a way to describe the total electric field as a sum of components, each of which is characterised by a discrete value of the related parameters. In the case of the frequency of the light field, this means we have to describe the field as a sum of monochromatic components. In the previous sections we have shown how this could be done in the special case of an initial monochromatic field that is subject to modulation: if the modulation index is small enough we can limit the amount of frequency components that we need to consider. In many cases it is actually sufficient to describe a modulation only by the interaction of the carrier at $\omega_{0}$ (the unmodulated field) and two sidebands with a frequency offset of $\pm \omega_{m}$ to the carrier. A beam given by the sum of three such components can be described by a complex vector:

$$
\vec{a}=\left(\begin{array}{c}
a\left(\omega_{0}\right) \\
a\left(\omega_{0}-\omega_{m}\right) \\
a\left(\omega_{0}+\omega_{m}\right)
\end{array}\right)=\left(\begin{array}{c}
a_{\omega 0} \\
a_{\omega 1} \\
a_{\omega 2}
\end{array}\right)
$$

with $\omega_{0}=\omega 0, \omega_{0}-\omega_{m}=\omega 1$ and $\omega_{0}+\omega_{m}=\omega 2$. In the case of a phase modulator that applies a modulation of small modulation index $m$ to an incoming light field $\vec{a}_{1}$, we can describe the coupling of the frequency component as follows:

$$
\begin{aligned}
& a_{2, \omega 0}=J_{0}(m) a_{1, \omega 0}+J_{1}(m) a_{1, \omega 1}+J_{-1}(m) a_{1, \omega 2} \\
& a_{2, \omega 1}=J_{0}(m) a_{1, \omega 1}+J_{-1}(m) a_{1, \omega 0} \\
& a_{2, \omega 2}=J_{0}(m) a_{1, \omega 2}+J_{1}(m) a_{1, \omega 0}
\end{aligned}
$$

which can be written in matrix form:

$$
\vec{a}_{2}=\left(\begin{array}{ccc}
J_{0}(m) & J_{1}(m) & J_{-1}(m) \\
J_{-1}(m) & J_{0}(m) & 0 \\
J_{1}(m) & 0 & J_{0}(m)
\end{array}\right) \vec{a}_{1} .
$$

And similarly, we can write the complete coupling matrix for the modulator component, for example, as

$$
\left(\begin{array}{c}
a_{2, w 0} \\
a_{2, w 1} \\
a_{2, w 2} \\
a_{4, w 0} \\
a_{4, w 1} \\
a_{4, w 2}
\end{array}\right)\left(\begin{array}{cccccc}
J_{0}(m) & J_{1}(m) & J_{-1}(m) & 0 & 0 & 0 \\
J_{-1}(m) & J_{0}(m) & 0 & 0 & 0 & 0 \\
J_{1}(m) & 0 & J_{0}(m) & 0 & 0 & 0 \\
0 & 0 & 0 & J_{0}(m) & J_{1}(m) & J_{-1}(m) \\
0 & 0 & 0 & J_{-1}(m) & J_{0}(m) & 0 \\
0 & 0 & 0 & J_{1}(m) & 0 & J_{0}(m)
\end{array}\right)\left(\begin{array}{l}
a_{1, w 0} \\
a_{1, w 1} \\
a_{1, w 2} \\
a_{3, w 0} \\
a_{3, w 1} \\
a_{3, w 2}
\end{array}\right)
$$




\subsection{Finesse examples}

\subsubsection{Modulation index}

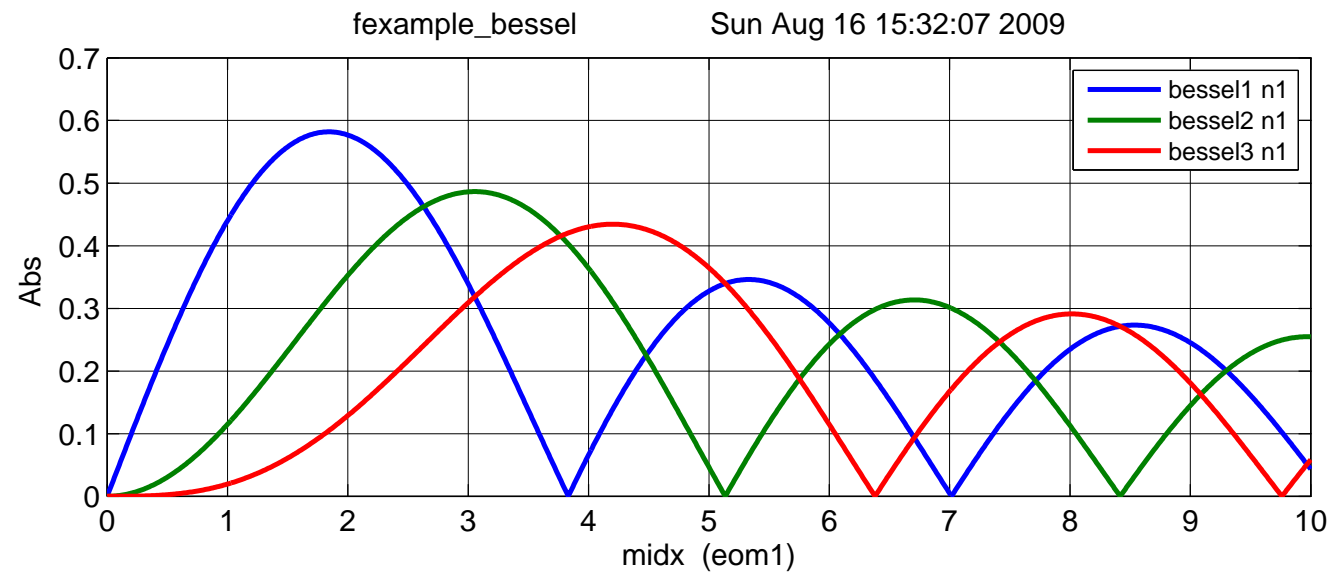

Figure 20: Finesse example: Modulation index.

This file demonstrates the use of a modulator. Phase modulation (with up to five higher harmonics is applied to a laser beam and amplitude detectors are used to measure the field at the first three harmonics. Compare this to Figure 16 as well.

Finesse input file for 'Modulation index'

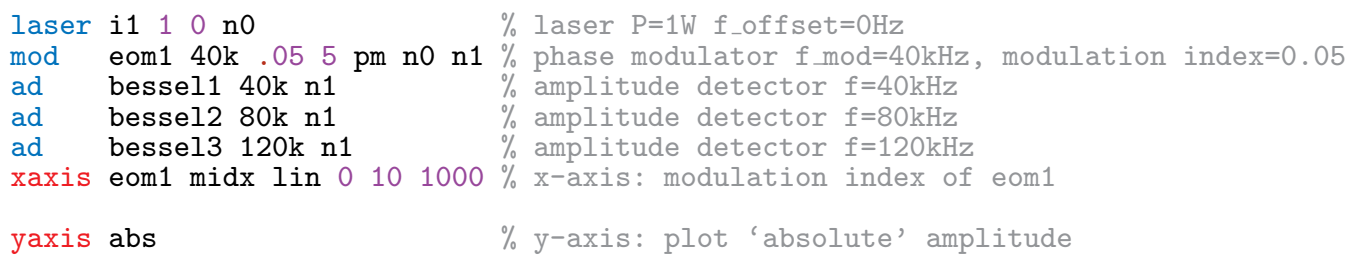

\subsubsection{Mirror modulation}

FINESSE offers two different types of modulators: the 'modulator' component shown in the example above, and the 'fsig' command, which can be used to apply a signal modulation to existing optical components. The main difference is that 'fsig' is meant to be used for transfer function computations. Consequently FINESSE discards all nonlinear terms, which means that the sideband amplitude is proportional to the signal amplitude and harmonics are not created.

Finesse input file for 'Mirror modulation'

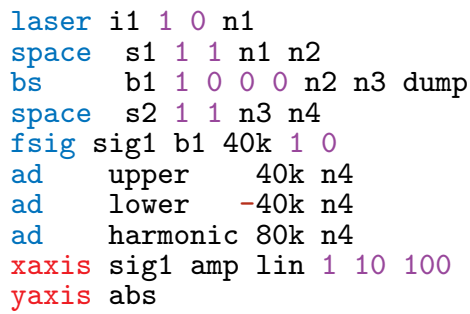

$\%$ laser $\mathrm{P}=1 \mathrm{~W}$ f_offset $=0 \mathrm{~Hz}$

$\%$ space of $1 \mathrm{~m}$ length

dump \% beam splitter as 'turning mirror', normal incidence

$\%$ another space of $1 \mathrm{~m}$ length

$\%$ signal modulation applied to beam splitter b1

$\%$ amplitude detector $\mathrm{f}=40 \mathrm{kHz}$

$\%$ amplitude detector $f=-40 \mathrm{kHz}$

$\%$ amplitude detector $\mathrm{f}=80 \mathrm{kHz}$

$\%$ x-axis: amplitude of signal modulation

$\%$ y-axis: plot 'absolute' amplitude 


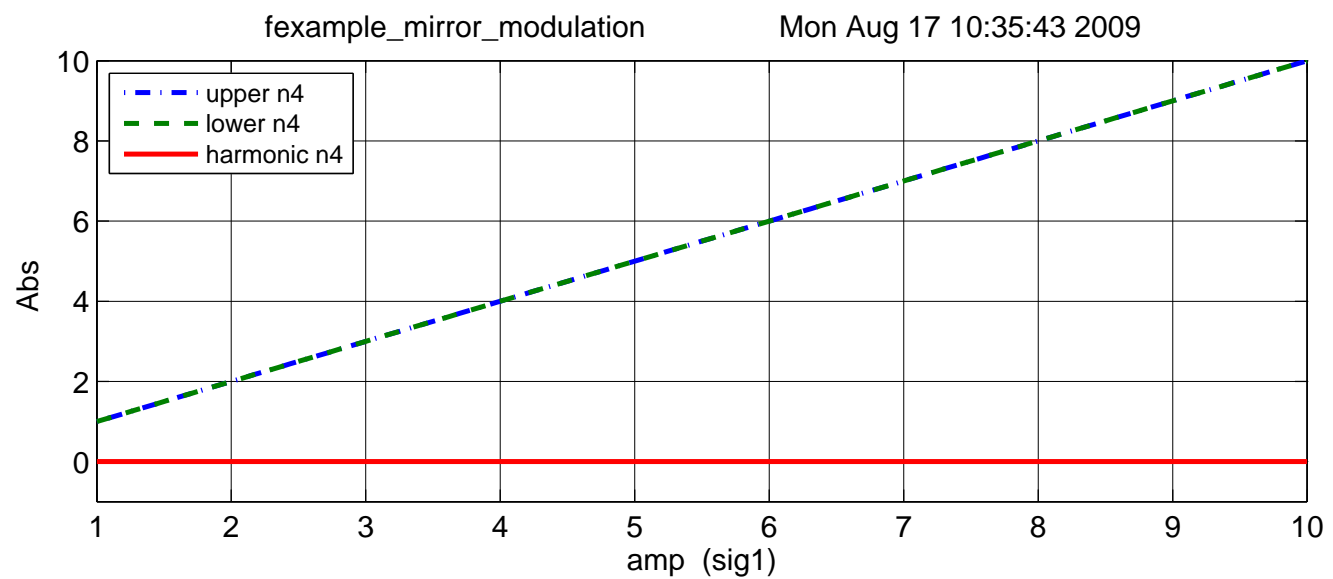

Figure 21: FINESSE example: Mirror modulation. 


\section{Optical Readout}

In previous sections we have dealt with the amplitude of light fields directly and also used the amplitude detector in the FINESSE examples. This is the advantage of a mathematical analysis versus experimental tests, in which only light intensity or light power can be measured directly. This section gives the mathematical details for modelling photo detectors.

The intensity of a field impinging on a photo detector is given as the magnitude of the Poynting vector, with the Poynting vector given as [58]

$$
\vec{S}=\vec{E} \times \vec{H}=\frac{1}{\mu_{0}} \vec{E} \times \vec{B}
$$

Inserting the electric and magnetic components of a plane wave, we obtain

$$
|\vec{S}|=\frac{1}{\mu_{0} c} E^{2}=c \epsilon_{0} E_{0}^{2} \cos ^{2}(\omega t)=\frac{c \epsilon_{0}}{2} E_{0}^{2}(1+\cos (2 \omega t)),
$$

with $\epsilon_{0}$ the electric permeability of vacuum and $c$ the speed of light.

The response of a photo detector is given by the total flux of effective radiation ${ }^{4}$ during the response time of the detector. For example, in a photo diode a photon will release a charge in the n-p junction. The response time is given by the time it takes for the charge to travel through the detector (and further time may be taken up in the electronic processing of the signal). The size of the photodiode and the applied bias voltage determine the travel time of the charges with typical values of approximately $10 \mathrm{~ns}$. Thus, frequency components faster than perhaps $100 \mathrm{MHz}$ are not resolved by a standard photodiode. For example, a laser beam with a wavelength of $\lambda=1064 \mathrm{~nm}$ has a frequency of $f=c / \lambda \approx 28210^{12} \mathrm{~Hz}=282 \mathrm{THz}$. Thus, the $2 \omega$ component is much too fast for the photo detector; instead, it returns the average power

$$
|\overline{\vec{S}}|=\frac{c \epsilon_{0}}{2} E_{0}^{2}
$$

In complex notation we can write

$$
|\overline{\vec{S}}|=\frac{c \epsilon_{0}}{2} E E^{*}
$$

However, for more intuitive results the light fields can be given in converted units, so that the light power can be computed as the square of the light field amplitudes. Unless otherwise noted, throughout this work the unit of light field amplitudes is $\sqrt{\text { watt }}$. Thus, the notation used in this document to describe the computation of the light power of a laser beam is

$$
P=E E^{*} \text {. }
$$

\subsection{Detection of optical beats}

What is usually called an optical beat or simply a beat is the sinusoidal behaviour of the intensity of two overlapping and coherent fields. For example, if we superpose two fields of slightly different frequency, we obtain

$$
\begin{aligned}
E= & E_{0} \cos \left(\omega_{1} t\right)+E_{0} \cos \left(\omega_{2} t\right) \\
P= & E^{2}=E_{0}^{2}\left(\cos ^{2}\left(\omega_{1} t\right)+\cos ^{2}\left(\omega_{2} t\right)+2 \cos \left(\omega_{1} t\right) \cos \left(\omega_{2} t\right)\right) \\
& =E_{0}^{2}\left(\cos ^{2}\left(\omega_{1} t\right)+\cos ^{2}\left(\omega_{2} t\right)+\cos \left(\omega_{+} t\right)+\cos \left(\omega_{-} t\right)\right),
\end{aligned}
$$

\footnotetext{
${ }^{4}$ The term effective refers to that amount of incident light, which is converted into photo-electrons that are then usefully extracted from the junction (i.e., do not recombine within the device). This fraction is usually referred to as quantum efficiency $\eta$ of the photodiode.
} 


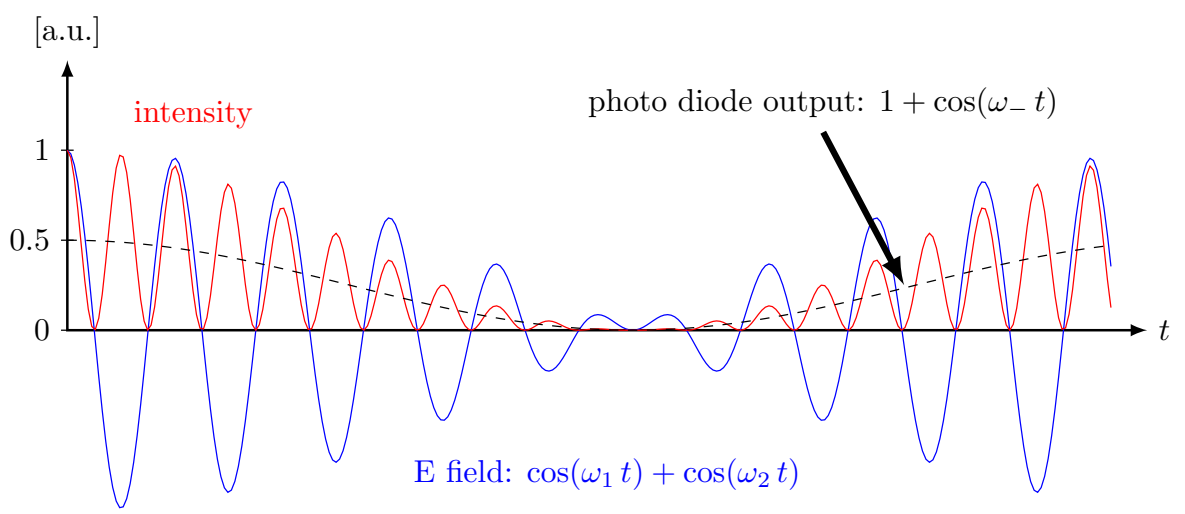

Figure 22: A beam with two frequency components hits the photo diode. Shown in this plot are the field amplitude, the corresponding intensity and the electrical output of the photodiode.

with $\omega_{+}=\omega_{1}+\omega_{2}$ and $\omega_{-}=\omega_{1}-\omega_{2}$. In this equation the frequency $\omega_{-}$can be very small and can then be detected with the photodiode as illustrated in Figure 22.

$$
P_{\text {diode }}=E_{0}^{2}\left(1+\cos \left(\omega_{-} t\right)\right)
$$

Using the same example photodiode as before: in order to be able to detect an optical beat $\omega_{-}$ would need to be smaller than $100 \mathrm{MHz}$. If we take two, sightly detuned Nd:YAG lasers with $f=282 \mathrm{THz}$, this means that the relative detuning of these lasers must be smaller than $10^{-7}$.

In general, for a field with several frequency components, the photodiode signal can be written as

$$
|E|^{2}=E \cdot E^{*}=\sum_{i=0}^{N} \sum_{j=0}^{N} a_{i} a_{j}^{*} e^{\mathrm{i}\left(\omega_{i}-\omega_{j}\right) t} .
$$

For example, if the photodiode signal is filtered with a low-pass filter, such that only the DC part remains, we can compute the resulting signal by looking for all components without frequency dependence. The frequency dependence vanishes when the frequency becomes zero, i.e., in all parts of Equation (75) with $\omega_{i}=\omega_{j}$. The output is a real number, calculated like this:

$$
x=\sum_{i} \sum_{j} a_{i} a_{j}^{*} \quad \text { with } \quad\left\{i, j \mid i, j \in\{0, \ldots, N\} \wedge \omega_{i}=\omega_{j}\right\} .
$$

\subsection{Signal demodulation}

A typical application of light modulation, is its use in a modulation-demodulation scheme, which applies an electronic demodulation to a photodiode signal. A 'demodulation' of a photodiode signal at a user-defined frequency $\omega_{x}$, performed by an electronic mixer and a low-pass filter, produces a signal, which is proportional to the amplitude of the photo current at DC and at the frequency $\omega_{0} \pm \omega_{x}$. Interestingly, by using two mixers with different phase offsets one can also reconstruct the phase of the signal, or to be precise the phase difference of the light at $\omega_{0} \pm \omega_{x}$ with respect to the carrier light. This feature can be very powerful for generating interferometer control signals.

Mathematically, the demodulation process can be described by a multiplication of the output with a cosine: $\cos \left(\omega_{x}+\varphi_{x}\right)\left(\varphi_{x}\right.$ is the demodulation phase), which is also called the 'local oscillator'. After the multiplication was performed only the DC part of the result is taken into account. The signal is

$$
S_{0}=|E|^{2}=E \cdot E^{*}=\sum_{i=0}^{N} \sum_{j=0}^{N} a_{i} a_{j}^{*} e^{\mathrm{i}\left(\omega_{i}-\omega_{j}\right) t} .
$$


Multiplied with the local oscillator it becomes

$$
\begin{aligned}
S_{1} & =S_{0} \cdot \cos \left(\omega_{x} t+\varphi_{x}\right)=S_{0} \frac{1}{2}\left(e^{\mathrm{i}\left(\omega_{x} t+\varphi_{x}\right)}+e^{-\mathrm{i}\left(\omega_{x} t+\varphi_{x}\right)}\right) \\
& =\frac{1}{2} \sum_{i=0}^{N} \sum_{j=0}^{N} a_{i} a_{j}^{*} e^{\mathrm{i}\left(\omega_{i}-\omega_{j}\right) t} \cdot\left(e^{\mathrm{i}\left(\omega_{x} t+\varphi_{x}\right)}+e^{-\mathrm{i}\left(\omega_{x} t+\varphi_{x}\right)}\right) .
\end{aligned}
$$

With $A_{i j}=a_{i} a_{j}^{*}$ and $e^{\mathrm{i} \omega_{i j} t}=e^{\mathrm{i}\left(\omega_{i}-\omega_{j}\right) t}$ we can write

$$
S_{1}=\frac{1}{2}\left(\sum_{i=0}^{N} A_{i i}+\sum_{i=0}^{N} \sum_{j=i+1}^{N}\left(A_{i j} e^{\mathrm{i} \omega_{i j} t}+A_{i j}^{*} e^{-\mathrm{i} \omega_{i j} t}\right)\right) \cdot\left(e^{\mathrm{i}\left(\omega_{x} t+\varphi_{x}\right)}+e^{-\mathrm{i}\left(\omega_{x} t+\varphi_{x}\right)}\right) .
$$

When looking for the DC components of $S_{1}$ we get the following [20]:

$$
\begin{aligned}
S_{1, \mathrm{DC}} & =\sum_{i j} \frac{1}{2}\left(A_{i j} e^{-\mathrm{i} \varphi_{x}}+A_{i j}^{*} e^{\mathrm{i} \varphi_{x}}\right) \quad \text { with } \quad\left\{i, j \mid i, j \in\{0, \ldots, N\} \wedge \omega_{i j}=\omega_{x}\right\} \\
& =\sum_{i j} \Re\left\{A_{i j} e^{-\mathrm{i} \varphi_{x}}\right\} .
\end{aligned}
$$

This would be the output of a mixer and a subsequent low-pass filter. The results for $\varphi_{x}=0$ and $\varphi_{x}=\pi / 2$ are called in-phase and in-quadrature, respectively (or also first and second quadrature). They are given by

$$
\begin{aligned}
S_{1, \mathrm{DC}, \text { phase }} & =\sum_{i j} \Re\left\{A_{i j}\right\}, \\
S_{1, \mathrm{DC}, \text { quad }} & =\sum_{i j} \Im\left\{A_{i j}\right\} .
\end{aligned}
$$

If only one mixer is used, the output is always real and is determined by the demodulation phase. However, with two mixers generating the in-phase and in-quadrature signals, it is possible to construct a complex number representing the signal amplitude and phase:

$$
z=\sum_{i j} a_{i} a_{j}^{*} \quad \text { with } \quad\left\{i, j \mid i, j \in\{0, \ldots, N\} \wedge \omega_{i j}=\omega_{x}\right\} .
$$

Often several sequential demodulations are applied in order to measure very specific phase information. For example, a double demodulation can be described as two sequential multiplications of the signal with two local oscillators and taking the DC component of the result. First looking at the whole signal, we can write:

$$
S_{2}=S_{0} \cdot \cos \left(\omega_{x} t+\varphi_{x}\right) \cos \left(\omega_{y} t+\varphi_{y}\right) .
$$

This can be written as

$$
\begin{aligned}
S_{2} & =S_{0} \frac{1}{2}\left(\cos \left(\omega_{y} t+\omega_{x} t+\varphi_{y}+\varphi_{x}\right)+\cos \left(\omega_{y} t-\omega_{x} t+\varphi_{y}-\varphi_{x}\right)\right) \\
& =S_{0} \frac{1}{2}\left(\cos \left(\omega_{+} t+\varphi_{+}\right)+\cos \left(\omega_{-} t+\varphi_{-}\right)\right),
\end{aligned}
$$

and thus reduced to two single demodulations. Since we now only care for the DC component we can use the expression from above (Equation (82)). These two demodulations give two complex numbers:

$$
\begin{aligned}
& z 1=\sum_{i j} A_{i j} \quad \text { with } \quad\left\{i, j \mid i, j \in\{0, \ldots, N\} \wedge \omega_{i}-\omega_{j}=\omega_{+}\right\} \\
& z 2=\sum_{i j} A_{k l} \quad \text { with } \quad\left\{k, l \mid k, l \in\{0, \ldots, N\} \wedge \omega_{k}-\omega_{l}=\omega_{-}\right\} .
\end{aligned}
$$

The demodulation phases are applied as follows to get a real output (two sequential mixers)

$$
x=\Re\left\{\left(z_{1} e^{-i \varphi_{x}}+z_{2} e^{\mathrm{i} \varphi_{x}}\right) e^{-\mathrm{i} \varphi_{y}}\right\} .
$$


In a typical setup, a user-defined demodulation phase for the first frequency (here $\varphi_{x}$ ) is given. If two mixers are used for the second demodulation, we can reconstruct the complex number

$$
z=z_{1} e^{-\mathrm{i} \varphi_{x}}+z_{2} e^{\mathrm{i} \varphi_{x}} .
$$

More demodulations can also be reduced to single demodulations as above. 


\subsection{Finesse examples}

\subsubsection{Optical beat}
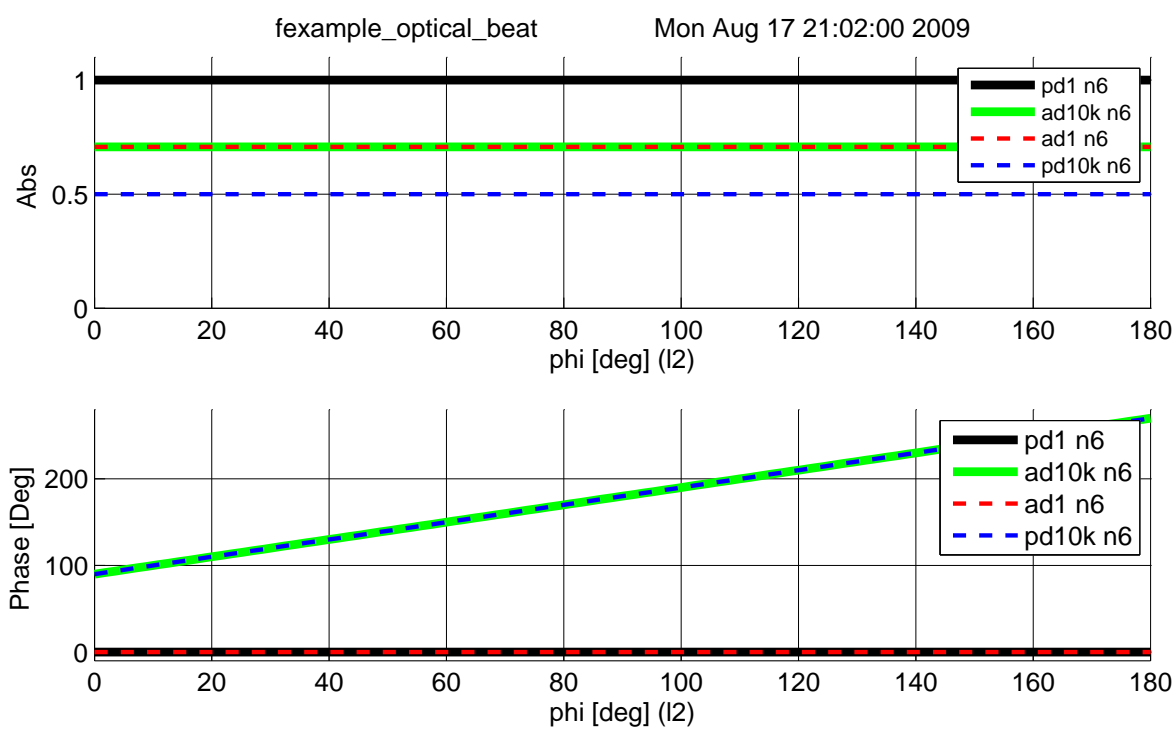

Figure 23: FinESSE example: Optical beat.

In this example two laser beams are superimposed at a 50:50 beam splitter. The beams have a slightly different frequency: the second beam has a $10 \mathrm{kHz}$ offset with respect to the first (and to the default laser frequency). The plot illustrates the output of four different detectors in one of the beam splitter output ports, while the phase of the second beam is tuned from $0^{\circ}$ to $180^{\circ}$. The photodiode 'pd1' shows the total power remaining constant at 1 . The amplitude detectors 'ad1' and 'ad10k' detect the laser light at $0 \mathrm{~Hz}$ (default frequency) and $10 \mathrm{kHz}$ respectively. Both show a constant absolute of $\sqrt{1 / 2}$ and the detector 'ad10k' tracks the tuning of the phase of the second laser beam. Finally, the detector 'pd10k' resembles a photodiode with demodulation at $10 \mathrm{kHz}$. In fact, this represents a photodiode and two mixers used to reconstruct a complex number as shown in Equation (82). One can see that the phase of the resulting electronic signal also directly follows the phase difference between the two laser beams.

\section{Finesse input file for 'Optical beat'}

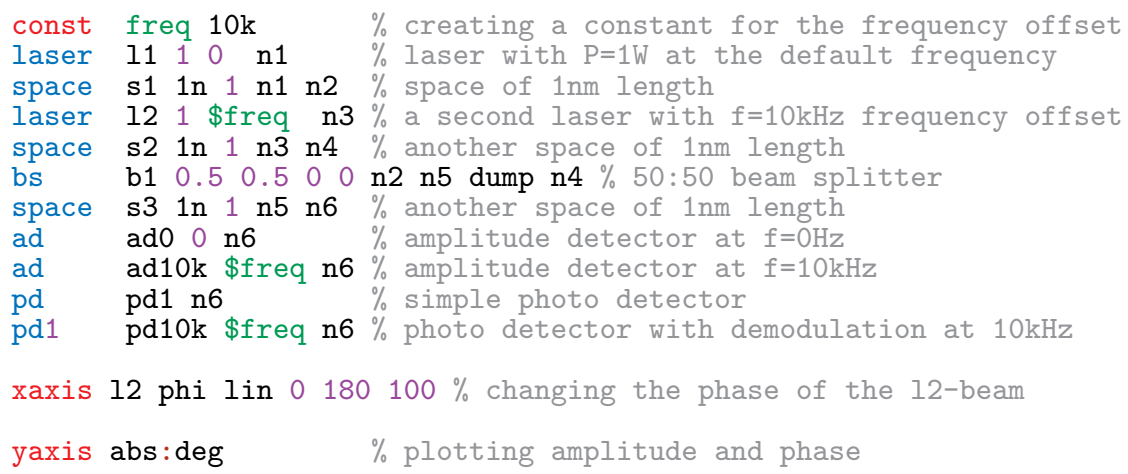




\section{$5 \quad$ Basic Interferometers}

The large interferometric gravitational-wave detectors currently in operation are based on two fundamental interferometer topologies: the Fabry-Pérot and the Michelson interferometer. The main instrument is very similar to the original interferometer concept used in the famous experiment by Michelson and Morley, published in 1887 [42]. The main difference is that modern instruments use laser light to illuminate the interferometer to achieve much higher accuracy. Already the first prototype by Forward and Weiss has thus achieved a sensitivity a million times better than Michelson's original instrument [18]. In addition, in current gravitational-wave detectors, the Michelson interferometer has been enhanced by resonant cavities, which in turn have been derived from the original idea for a spectroscopy standard published by Fabry and Pérot in 1899 [16]. The following section will describe the fundamental properties of the Fabry-Pérot interferometer and the Michelson interferometer. A thorough understanding of these basic instruments is essential for the study of the high-precision interferometers used for gravitational-wave detection.

\subsection{The two-mirror cavity: a Fabry-Pérot interferometer}

We have computed the field amplitudes in a linear two-mirror cavity, also called Fabry-Pérot interferometer, in Section 2.2. In order to understand the features of this optical instrument it is of interest to have a closer look at the power circulation in the cavity. A typical optical layout is shown in Figure 24: two parallel mirrors form the Fabry-Pérot cavity. A laser beam is injected through the first mirror (at normal incidence).

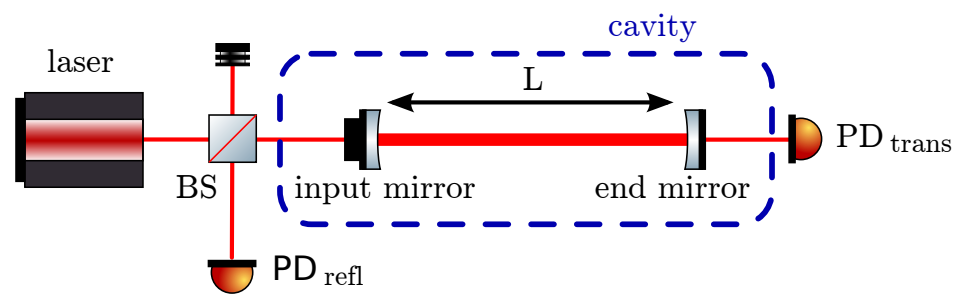

Figure 24: Typical optical layout of a two-mirror cavity, also called a Fabry-Pérot interferometer. Two mirrors form the Fabry-Pérot interferometer, a laser beam is injected through one of the mirrors and the reflected and transmitted light can be detected by photo detectors.

The behaviour of the (ideal) cavity is determined by the length of the cavity $L$, the wavelength of the laser $\lambda$ and the reflectivity and transmittance of the mirrors. Assuming an input power of $\left|a_{0}\right|^{2}=1$, we obtain

$$
P_{1}=\left|a_{1}\right|^{2}=\frac{T_{1}}{1+R_{1} R_{2}-2 r_{1} r_{2} \cos (2 k L)},
$$

with $k=2 \pi / \lambda, P, T=t^{2}$ and $R=r^{2}$, as defined in Section 1.4. Similarly we could compute the transmission of the optical system as the input-output ratio of the field amplitudes. For example,

$$
\frac{a_{2}}{a_{0}}=\frac{-t_{1} t_{2} \exp (-\mathrm{i} k L)}{1-r_{1} r_{2} \exp (-\mathrm{i} 2 k L)}
$$

is the frequency-dependent transfer function of the cavity in transmission (the frequency dependency is hidden inside the $k=2 \pi f / c)$.

Figure 25 shows a plot of the circulating light power $P_{1}$ over the laser frequency. The maximum power is reached when the cosine function in the denominator becomes equal to one, i.e., at

Living Reviews in Relativity

http: //www . livingreviews . org/lrr-2010-1 


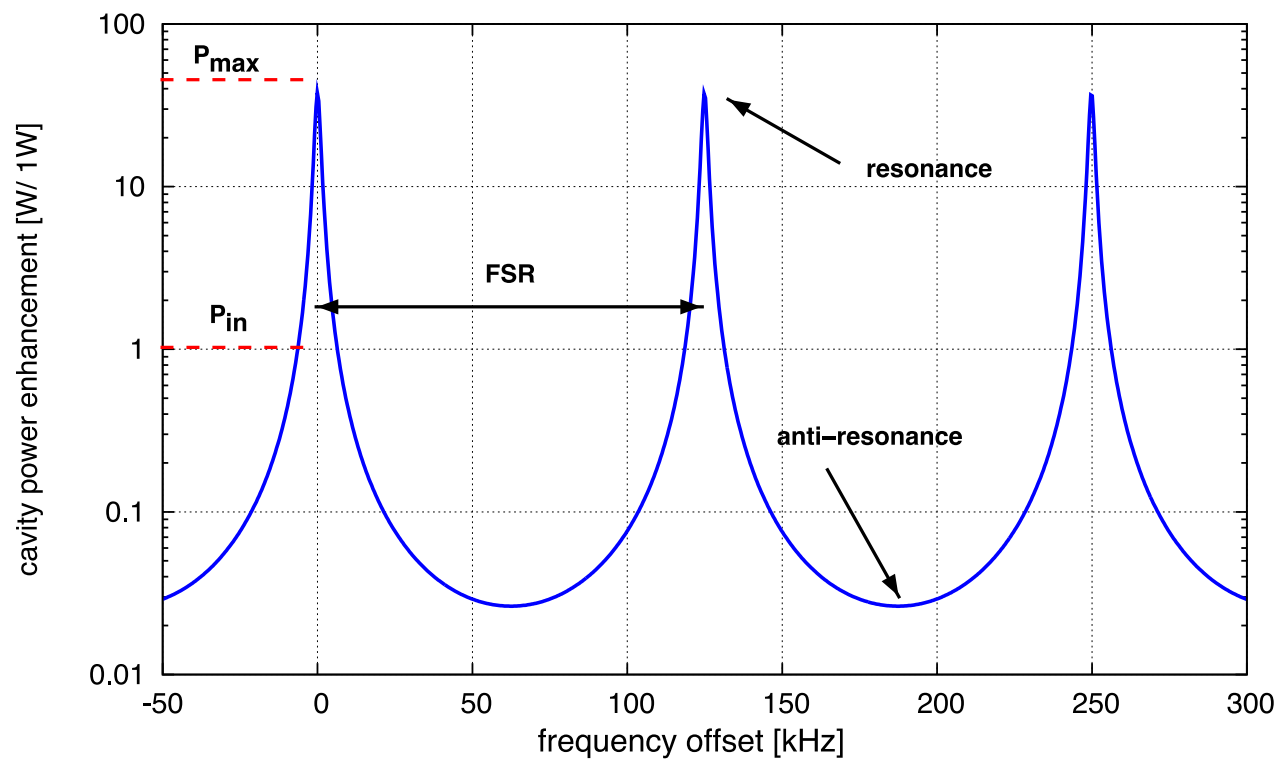

Figure 25: Power enhancement in a two-mirror cavity as a function of the laser-light frequency. The peaks marks the resonances of the cavity, i.e., modes of operation in which the injected light is resonantly enhanced. The frequency distance between two peaks is called free-spectral range (FSR).

$k L=N \pi$ with $N$ an integer. This is called the cavity resonance. The lowest power values are reached at anti-resonance when $k L=(N+1 / 2) \pi$. We can also rewrite

$$
2 k L=\omega \frac{2 L}{c}=2 \pi f \frac{2 L}{c}=\frac{2 \pi f}{\mathrm{FSR}},
$$

with FSR being the free-spectral range of the cavity as shown in Figure 25. Thus, it becomes clear that resonance is reached for laser frequencies

$$
f_{r}=N \cdot \mathrm{FSR}
$$

where $N$ is an integer.

Another characteristic parameter of a cavity is its linewidth, usually given as full width at half maximum (FWHM) or its pole frequency, $f_{p}$. In order to compute the linewidth we have to ask at which frequency the circulating power becomes half the maximum:

$$
\left|a_{1}\left(f_{p}\right)\right|^{2} \stackrel{!}{=} \frac{1}{2}\left|a_{1, \max }\right|^{2} .
$$

This results in the following expression for the full linewidth:

$$
\mathrm{FWHM}=2 f_{p}=\frac{2 \mathrm{FSR}}{\pi} \arcsin \left(\frac{1-r_{1} r_{2}}{2 \sqrt{r_{1} r_{2}}}\right) \text {. }
$$

The ratio of the linewidth and the free spectral range is called the finesse of a cavity:

$$
F=\frac{\mathrm{FSR}}{\mathrm{FWHM}}=\frac{\pi}{2 \arcsin \left(\frac{1-r_{1} r_{2}}{2 \sqrt{r_{1} r_{2}}}\right)} .
$$

In the case of high finesse, i.e., $r_{1}$ and $r_{2}$ are close to 1 we can use the fact that the argument of the arcsin function is small and make the approximation

$$
F \approx \frac{\pi \sqrt{r_{1} r_{2}}}{1-r_{1} r_{2}} \approx \frac{\pi}{1-r_{1} r_{2}} .
$$



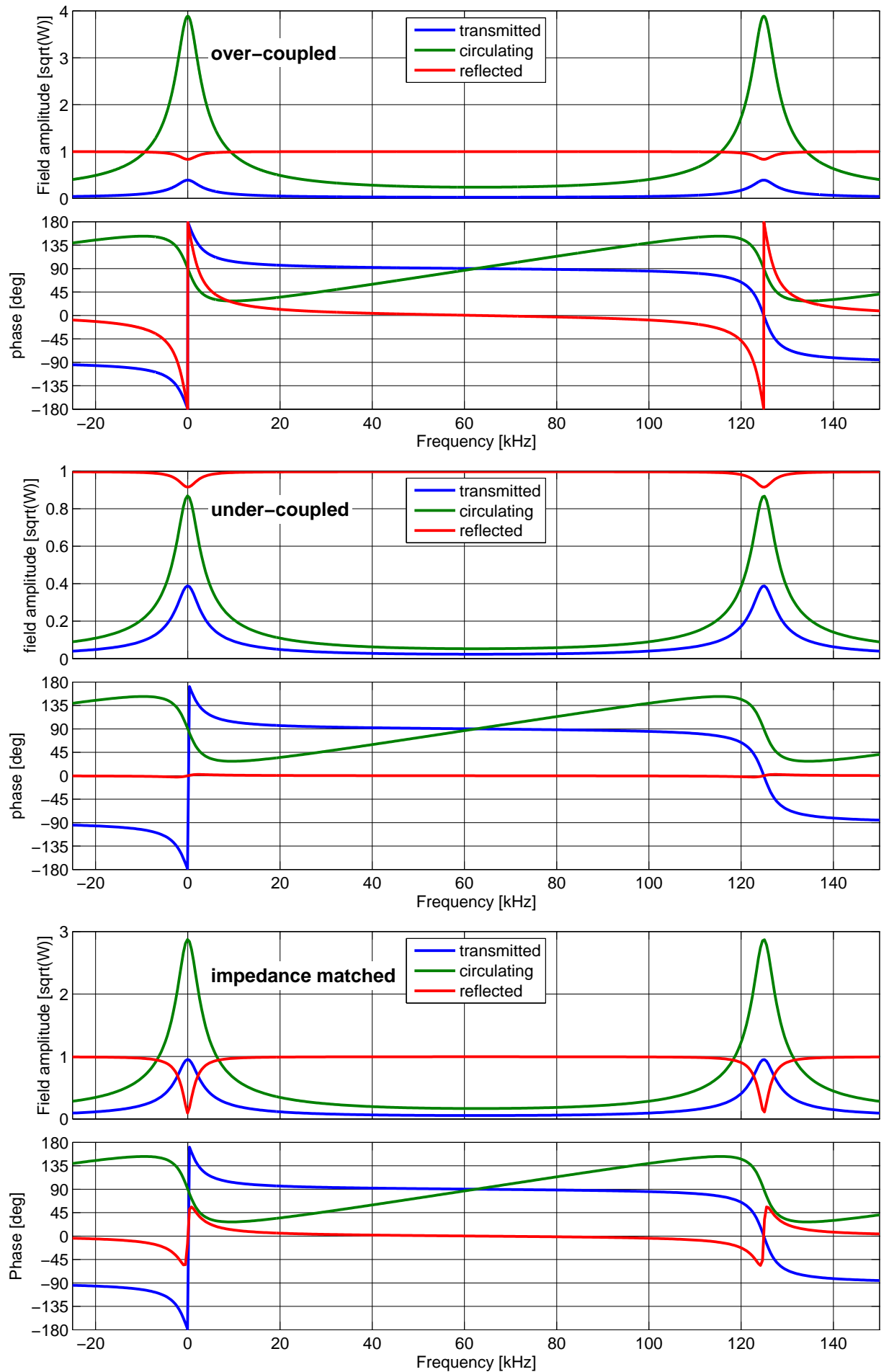

Figure 26: This figure compares the fields reflected by, transmitted by and circulating in a Fabry-Pérot cavity for the three different cases: over-coupled, under-coupled and impedance matched cavity (in all cases $T_{1}+T_{2}=0.2$ and the round-trip loss is $1 \%$ ). The traces show the phase and amplitude of the electric field as a function of laser frequency detuning. 


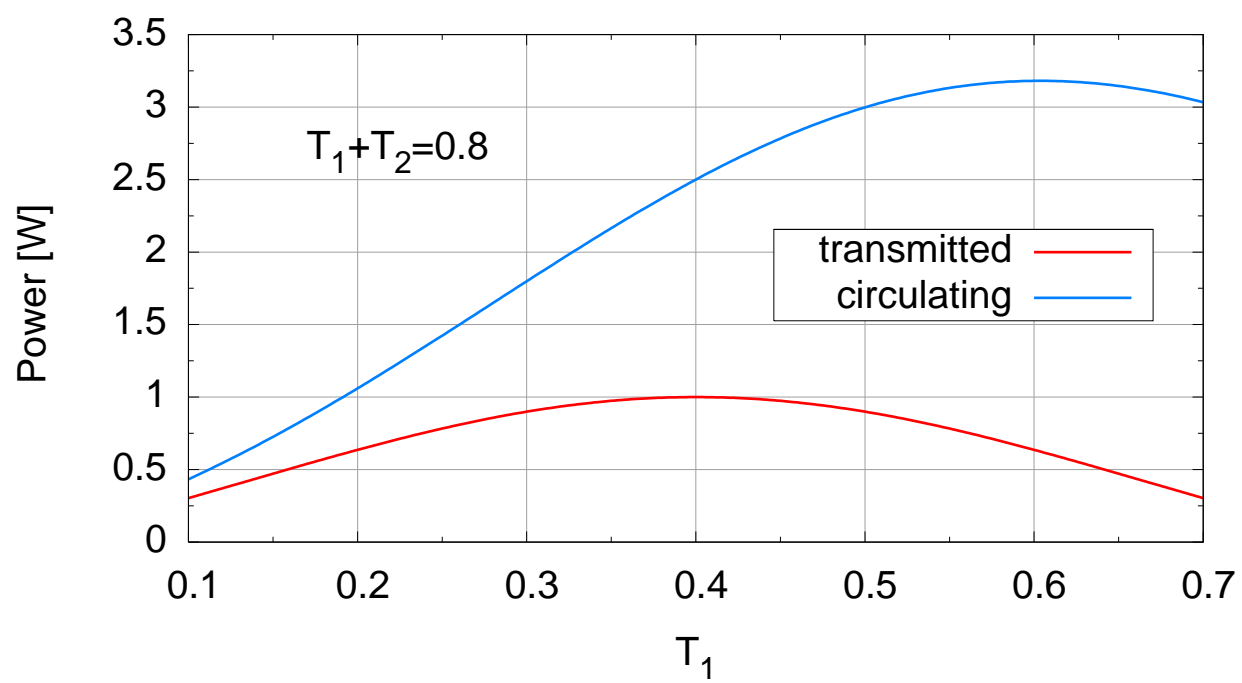

Figure 27: Power transmitted and circulating in a two mirror cavity with input power $1 \mathrm{~W}$. The mirror transmissions are set such that $T_{1}+T_{2}=0.8$ and the reflectivities of both mirrors are set as $R=1-T$. The cavity is undercoupled for $T_{1}<0.4$, impedance matched at $T_{1}=T_{2}=0.4$ and overcoupled for $T_{1}>0.4$. The transmission is maximised in the impedance-matched case and falls similarly for over or undercoupled settings. However, the circulating power (and any resonance performance of the cavity) is much larger in the overcoupled case.

The behaviour of a two mirror cavity depends on the length of the cavity (with respect to the frequency of the laser) and on the reflectivities of the mirrors. Regarding the mirror parameters one distinguishes three $\operatorname{cases}^{5}$ :

- when $T_{1}<T_{2}$ the cavity is called undercoupled

- when $T_{1}=T_{2}$ the cavity is called impedance matched

- when $T_{1}>T_{2}$ the cavity is called overcoupled

The differences between these three cases can seem subtle mathematically but have a strong impact on the application of cavities in laser systems. One of the main differences is the phase evolution of the light fields, which is shown in Figure 26. The circulating power shows that the resonance effect is better used in over-coupled cavities; this is illustrated in Figure 27, which shows the transmitted and circulating power for the three different cases. Only in the impedancematched case can the cavity transmit (on resonance) all the incident power. Given the same total transmission $T_{1}+T_{2}$, the overcoupled case allows for the largest circulating power and thus a stronger 'resonance effect' of the cavity, for example, when the cavity is used as a mode filter. Hence, most commonly used cavities are impedance matched or overcoupled.

\footnotetext{
${ }^{5}$ Please note that in the presence of losses the coupling is defined with respect to the transmission and losses. In particular, the impedance-matched case is defined as $T_{1}=T_{2} \cdot$ Loss, so that the input power transmission exactly matches the light power lost in one round-trip.
} 


\subsection{Michelson interferometer}

We came across the Michelson interferometer in Section 2.4 when we discussed the phase relation at a beam splitter. The typical optical layout of the Michelson interferometer is shown again in Figure 28: a laser beam is split by a beam splitter and send along two perpendicular interferometer arms. The four directions seen from the beam splitter are called North, East, West and South. The ends of these arms (North and East) are marked by highly reflective end mirrors, which reflect the beams back into themselves so that they can be recombined by the beam splitter. Generally, the Michelson interferometer has two outputs, namely the so far unused beam splitter port (South) and the input port (West). Both output ports can be used to obtain interferometer signals, however, most setups are designed such that the signals with high signal-to-noise ratios are detected in the South port.

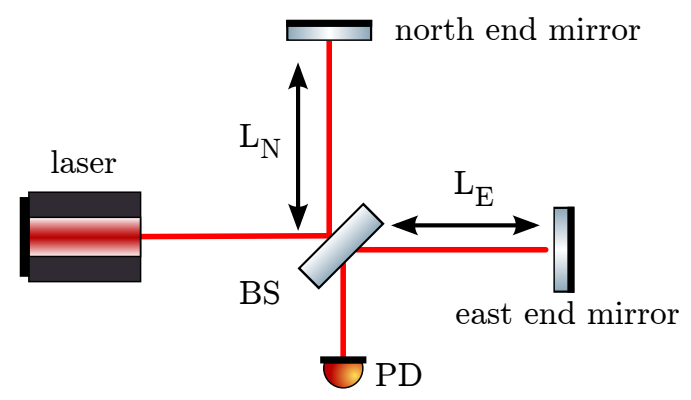

Figure 28: Typical optical layout of a Michelson interferometer: a laser beam is split into two and sent along two perpendicular interferometer arms. We will label the directions in a Michelson interferometer as North, East, West and South in the following. The end mirrors reflect the beams such that they are recombined at the beam splitter. The South and West ports of the beam splitter are possible output port, however, in many cases, only the South port is used.

The Michelson interferometer output is determined by the laser wavelength $\lambda$, the reflectivity and transmittance of the beam splitter and the end mirrors, and the length of the interferometer arms. In many cases the end mirrors are highly reflective and the beam splitter ideally a 50:50 beam splitter. In that case, we can compute the output for a monochromatic field as shown in Section 2.4. Using Equation (20) we can write the field in the South port as

$$
E_{S}=E_{0} \frac{\mathrm{i}}{2}\left(e^{\mathrm{i} 2 k L_{N}}+e^{\mathrm{i} 2 k L_{E}}\right) .
$$

We define the common arm length and the arm-length difference as

$$
\begin{aligned}
& \bar{L}=\frac{L_{N}+L_{E}}{2} \\
& \Delta L=L_{N}-L_{E},
\end{aligned}
$$

which yield $2 L_{N}=2 \bar{L}+\Delta L$ and $2 L_{E}=2 \bar{L}-\Delta L$. Thus, we can further simplify to get

$$
E_{S}=E_{0} \frac{\mathrm{i}}{2} e^{\mathrm{i} 2 k \bar{L}}\left(e^{\mathrm{i} k \Delta L}+e^{-\mathrm{i} k \Delta L}\right)=E_{0} \mathrm{i} e^{\mathrm{i} 2 k \bar{L}} \cos (k \Delta L) .
$$

The photo detector then produces a signal proportional to

$$
S=E_{S} E_{S}^{*}=P_{0} \cos ^{2}(k \Delta L)=P_{0} \cos ^{2}(2 \pi \Delta L / \lambda) .
$$

This signal is depicted in Figure 29; it shows that the power in the South port changes between zero and the input power with a period of $\Delta L / \lambda=0.5$. The tuning at which the output power 


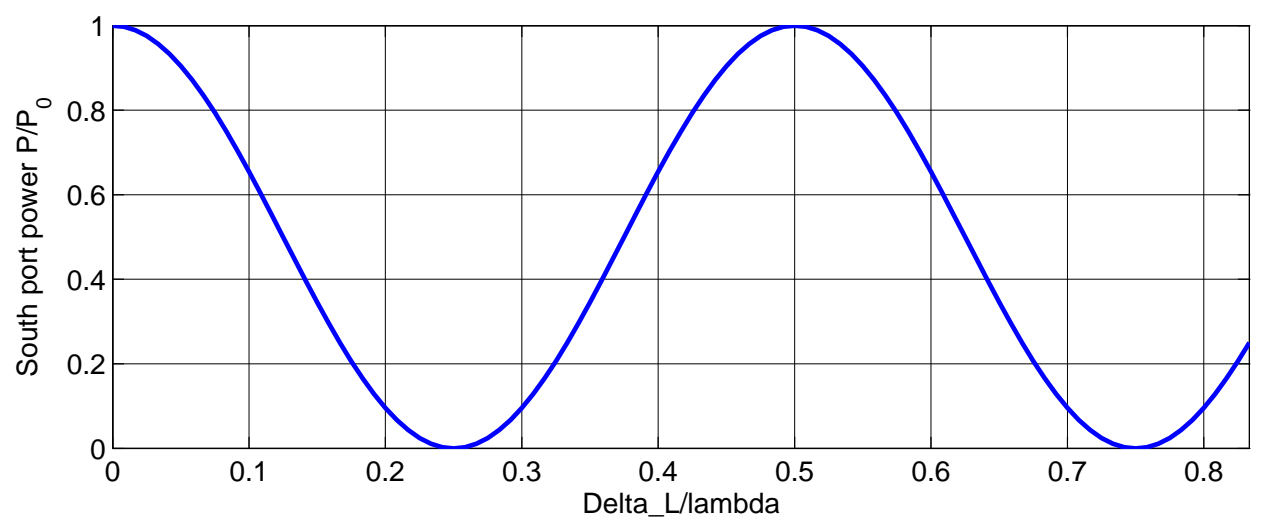

Figure 29: Power in the South port of a symmetric Michelson interferometer as a function of the arm length difference $\Delta L$.

drops to zero is called the dark fringe. Current interferometric gravitational-wave detectors operate their Michelson interferometer at or near the dark fringe.

The above seems to indicate that the macroscopic arm-length difference plays no role in the Michelson output signal. However, this is only correct for a monochromatic laser beam with infinite coherence length. In real interferometers care must be taken that the arm-length difference is well below the coherence length of the light source. In gravitational-wave detectors the macroscopic arm-length difference is an important design feature; it is kept very small in order to reduce coupling of laser noise into the output but needs to retain a finite size to allow the transfer of phase modulation sidebands from the input to the output port; this is illustrated in the FINESSE example below and will be covered in detail in Section 6.4. 


\subsection{Finesse examples}

\subsubsection{Michelson power}

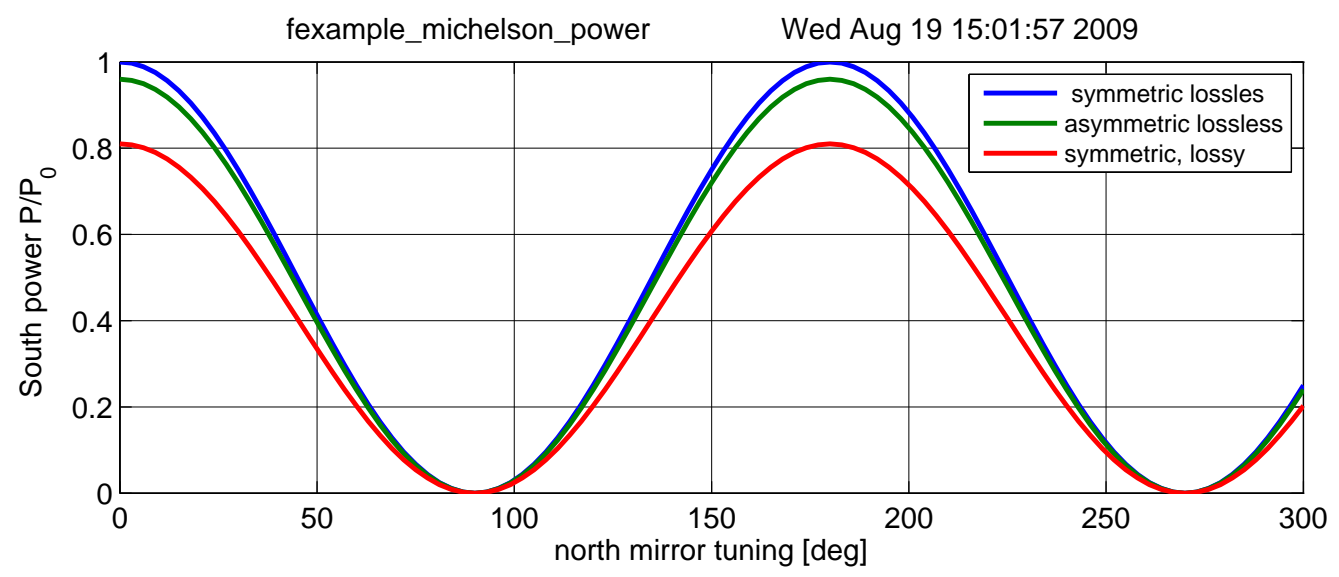

Figure 30: FINESSE example: Michelson power.

The power in the South port of a Michelson detector varies as the cosine squared of the microscopic arm length difference. The maximum output can be equal to the input power, but only if the Michelson interferometer is symmetric and lossless. The tuning for which the South port power is zero is referred to as the dark fringe.

Finesse input file for 'Michelson power'

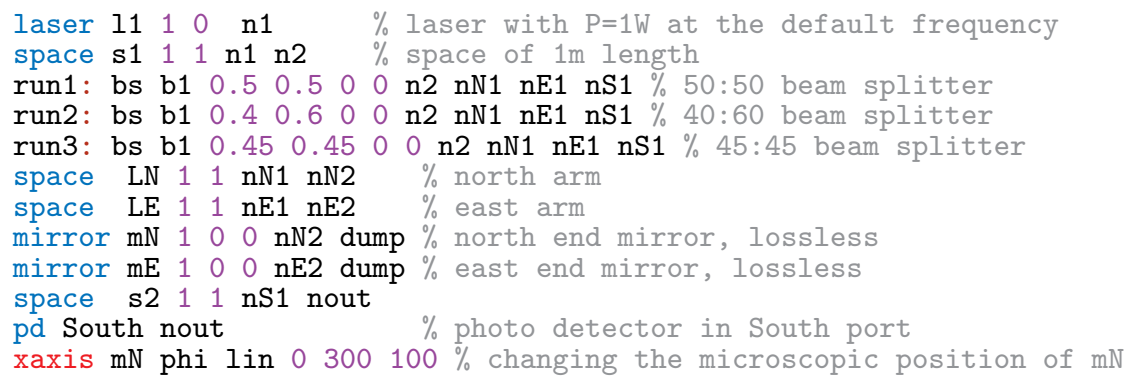

\subsubsection{Michelson modulation}

This example demonstrates how a macroscopic arm length difference can cause different 'dark fringe' tuning for injected fields with different frequencies. In this case, some of the $10 \mathrm{MHz}$ modulation sidebands are transmitted when the interferometer is tuned to a dark fringe for the carrier light. This effect can be used to separate light fields of different frequencies. It is also the cause for transmission of laser noise (especially frequency noise) into the Michelson output port when the interferometer is not perfectly symmetric.

\section{Finesse input file for 'Michelson modulation'}

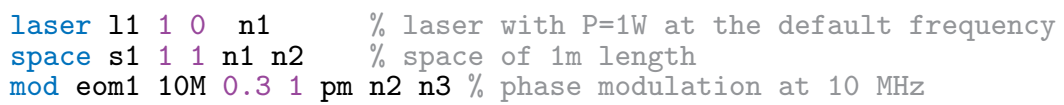




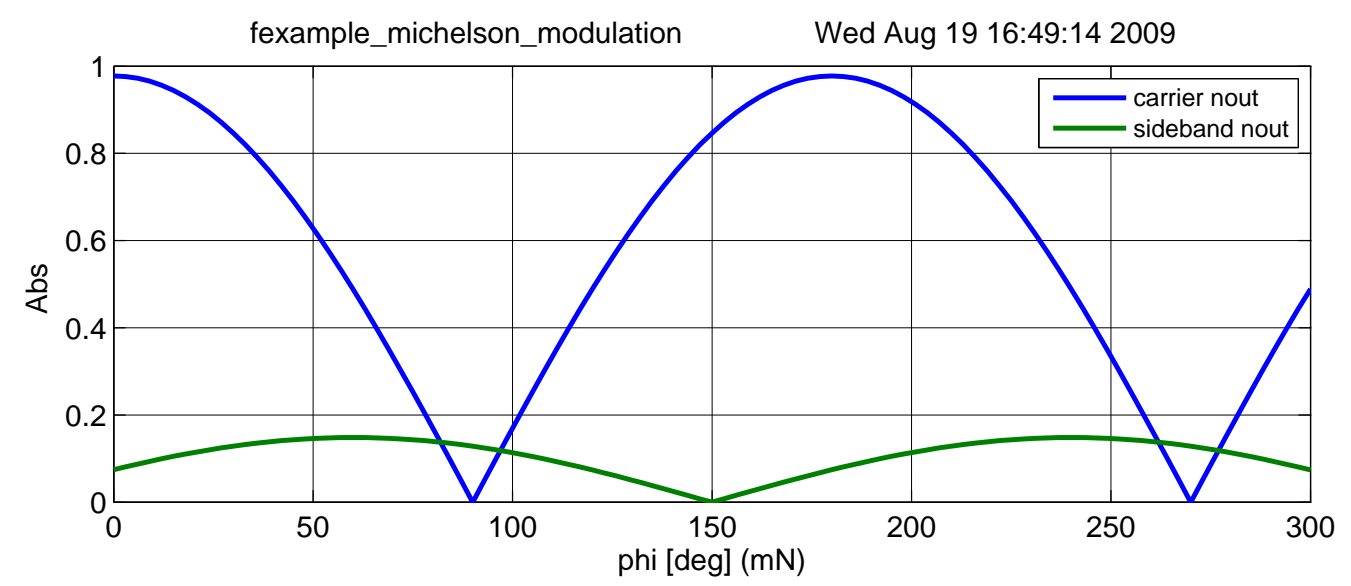

Figure 31: FinESSE example: Michelson modulation.

space s2 $11 \mathrm{n} 3 \mathrm{n} 4 \quad \%$ another space of $1 \mathrm{~m}$ length

bs b1 0.50 .500 n4 nN1 nE1 nS1 \% 50:50 beam splitter

space LN $1001 \mathrm{nN} 1 \mathrm{nN2} \%$ north arm

space LE $1101 \mathrm{nE} 1 \mathrm{nE2} \%$ east arm

mirror $\mathrm{mN} 100 \mathrm{nN} 2$ dump \% north end mirror, lossless

mirror mE 100 nE2 dump \% east end mirror, lossless

space s3 11 nS1 nout

ad carrier 0 nout

$\%$ amplitude detector for carrier field

ad sideband 10M nout $\%$ amplitude detector for $+10 \mathrm{MHz}$ sideband

xaxis mN phi lin $0300100 \%$ changing the microscopic position of $\mathrm{mN}$ 


\section{Interferometric Length Sensing and Control}

In this section we introduce interferometers as length sensing devices. In particular, we explain how the Fabry-Pérot interferometer and the Michelson interferometer can be used for high-precision measurements and that both require a careful control of the base length (which is to be measured) in order to yield their large sensitivity. In addition, we briefly introduce the general concepts of error signals and transfer functions, which are used to describe most essential features of length sensing and control.

\subsection{Error signals and transfer functions}

In general, we will call an error signal any measured signal suitable for stabilising a certain experimental parameter $p$ with a servo loop. The aim is to maintain the variable $p$ at a user-defined value, the operating point, $p_{0}$. Therefore, the error signal must be a function of the parameter $p$. In most cases it is preferable to have a bipolar signal with a zero crossing at the operating point. The slope of the error signal at the operating point is a measure of the 'gain' of the sensor (which in the case of interferometers is a combination of optics and electronics).

Transfer functions describe the propagation of a periodic signal through a plant and are usually given as plots of amplitude and phase over frequency. By definition a transfer function describes only the linear coupling of signals inside a system. This means a transfer function is independent of the actual signal size. For small signals or small deviations, most systems can be linearised and correctly described by transfer functions.

Experimentally, network analysers are commonly used to measure a transfer function: one connects a periodic signal (the source) to an actuator of the plant (which is to be analysed) and to an input of the analyser. A signal from a sensor that monitors a certain parameter of the plant is connected to the second analyser input. By mixing the source with the sensor signal the analyser can determine the amplitude and phase of the input signal with respect to the source (amplitude equals one and the phase equals zero when both signals are identical).

Mathematically, transfer functions can be modeled similarly: applying a sinusoidal signal $\sin \left(\omega_{s} t\right)$ to the interferometer, e.g., as a position modulation of a cavity mirror, will create phase modulation sidebands with a frequency offset of $\pm \omega_{s}$ to the carrier light. If such light is detected in the right way by a photodiode, it will include a signal at the frequency component $\omega_{s}$, which can be extracted, for example, by means of demodulation (see Section 4.2).

Transfer functions are of particular interest in relation to error signals. Typically a transfer function of the error signal is required for the design of the respective electronic servo. A 'transfer function of the error signal' usually refers to a very specific setup: the system is held at its operating point, such that, on average, $\bar{p}=p_{0}$. A signal is applied to the system in the form of a very small sinusoidal disturbance of $p$. The transfer function is then constructed by computing for each signal frequency the ratio of the error signal and the injected signal. Figure 32 shows an example of an error signal and its corresponding transfer function. The operating point shall be at

$$
x_{\mathrm{d}}=0 \quad \text { and } \quad x_{\mathrm{EP}}\left(x_{\mathrm{d}}=0\right)=0
$$

The optical transfer function $T_{\mathrm{opt}, \mathrm{x}_{\mathrm{d}}}$ with respect to this error signal is defined by

$$
\widetilde{x}_{\mathrm{EP}}(f)=T_{\mathrm{opt}, \mathrm{x}_{\mathrm{d}}} T_{\mathrm{det}} \widetilde{x}_{d}(f),
$$

with $T_{\text {det }}$ as the transfer function of the sensor. In the following, $T_{\text {det }}$ is assumed to be unity. At the zero crossing the slope of the error signal represents the magnitude of the transfer function for low frequencies:

$$
\left|\frac{d x_{\mathrm{EP}}}{d x_{\mathrm{d}}}\right|_{\mid x_{\mathrm{d}}=0}=\left|T_{\mathrm{opt}, \mathrm{x}_{\mathrm{d}}}\right|_{\mid f \rightarrow 0}
$$

Living Reviews in Relativity

http: //www. livingreviews.org/lrr-2010-1 

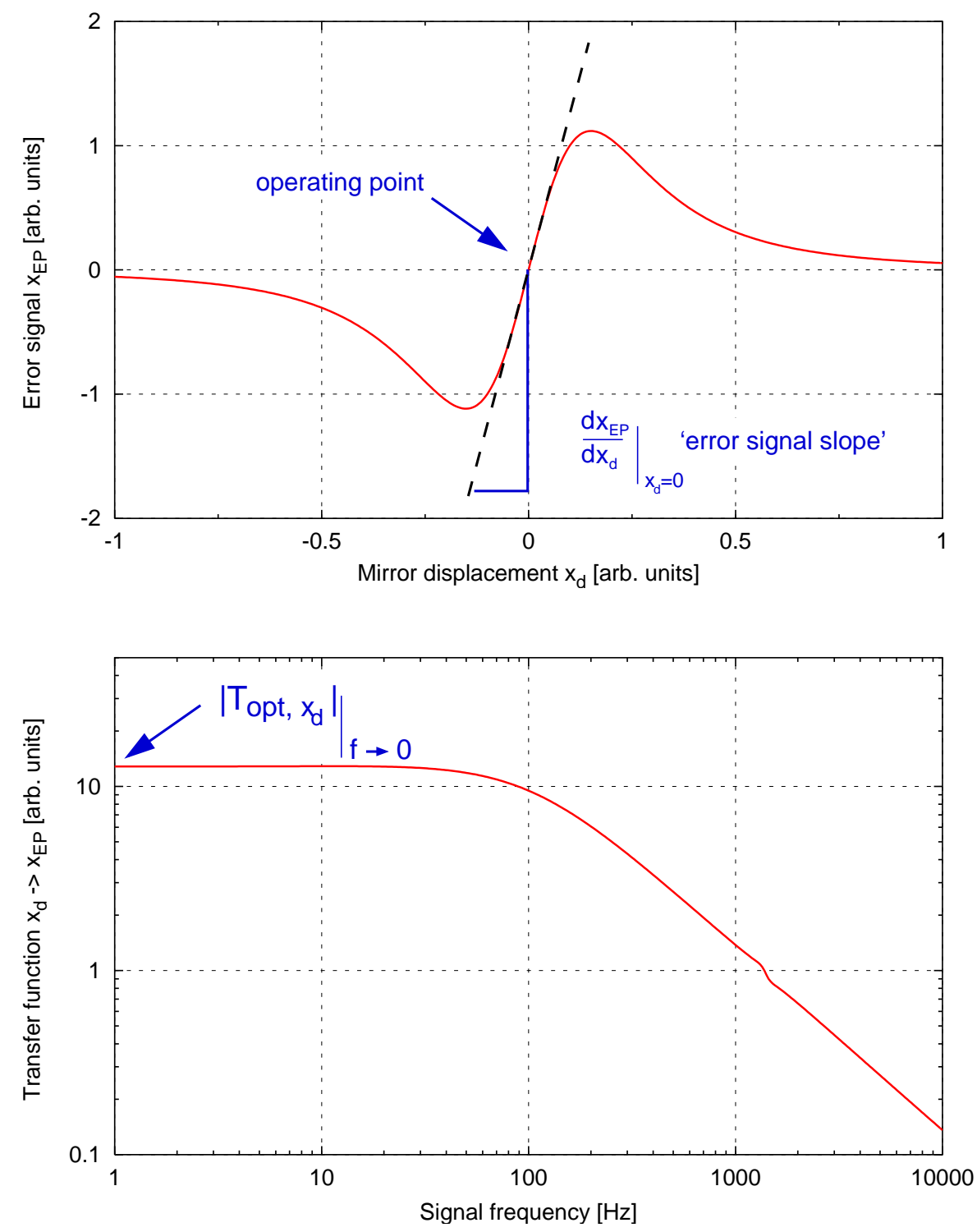

Figure 32: Example of an error signal: the top graph shows the electronic interferometer output signal as a function of mirror displacement. The operating point is given as the zero crossing, and the error-signal slope is defined as the slope at the operating point. The right graph shows the magnitude of the transfer function mirror displacement $\rightarrow$ error signal. The slope of the error signal (left graph) is equal to the low frequency limit of the transfer function magnitude (see Equation (102)). 
The quantity above will be called the error-signal slope in the following text. It is proportional to the optical gain $\left|T_{\mathrm{opt}, x_{\mathrm{d}}}\right|$, which describes the amplification of the gravitational-wave signal by the optical instrument.

\subsection{Fabry-Pérot length sensing}

In Figure 25 we have plotted the circulating power in a Fabry-Pérot cavity as a function of the laser frequency. The steep features in this plot indicate that such a cavity can be used to measure changes in the laser frequency. From the equation for the circulating power (see Equation (88)),

$$
P_{1} / P_{0}=\frac{T_{1}}{1+R_{1} R_{2}-2 r_{1} r_{2} \cos (2 k L)}=\frac{T_{1}}{d}
$$

we can see that the actual frequency dependence is given by the $\cos (2 k L)$ term. Writing this term as

$$
\cos (2 k L)=\cos \left(2 \pi \frac{L f}{c}\right),
$$

we can highlight the fact that the cavity is in fact a reference for the laser frequency in relation to the cavity length. If we know the cavity length very well, a cavity should be a good instrument to measure the frequency of a laser beam. However, if we know the laser frequency very accurately, we can use an optical cavity to measure a length. In the following we will detail the optical setup and behaviour of a cavity used for a length measurement. The same reasoning applies for frequency measurements. If we make use of the resonant power enhancement of the cavity to measure the cavity length, we can derive the sensitivity of the cavity from the differentiation of Equation (88), which gives the slope of the trace shown in Figure 25,

$$
\frac{d P_{1} / P_{0}}{d L}=\frac{-4 T_{1} r_{1} r_{2} k \sin (2 k L)}{d^{2}}
$$

with $d$ as defined in Equation (103). This is plotted in Figure 33 together with the cavity power as a function of the cavity tuning. From Figure 33 we can deduce a few key features of the cavity:

- The cavity must be held as near as possible to the resonance for maximum sensitivity. This is the reason that active servo control systems play an important role in modern laser interferometers.

- If we want to use the power directly as an error signal for the length, we cannot use the cavity directly on resonance because there the optical gain is zero. A suitable error signal (i.e., a bipolar signal) can be constructed by adding an offset to the light power signal. A control system utilising this method is often called DC-lock or offset-lock. However, we show below that more elegant alternative methods for generating error signals exist.

- The differentiation of the cavity power looks like a perfect error signal for holding the cavity on resonance. A signal proportional to such differentiation can be achieved with a modulationdemodulation technique. 

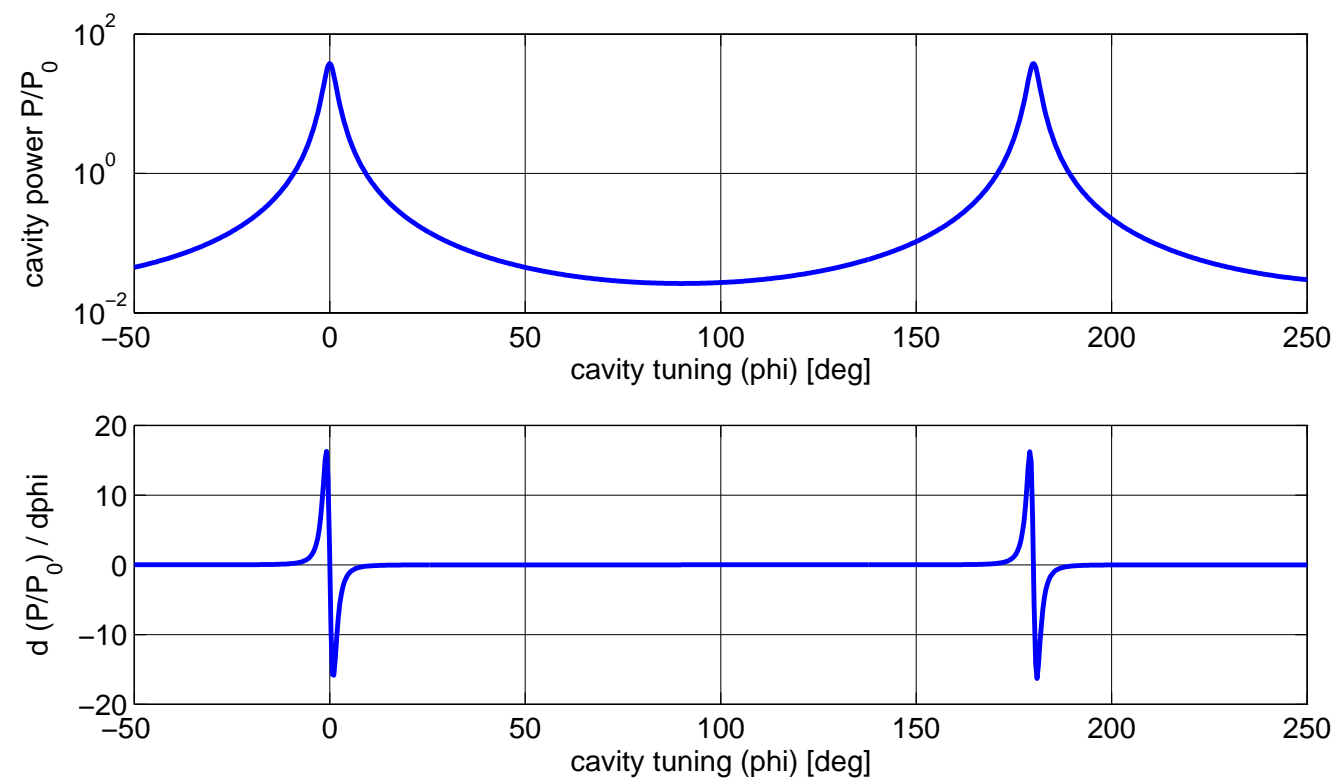

Figure 33: The top plot shows the cavity power as a function of the cavity tuning. A tuning of $360^{\circ}$ refers to a change in the cavity length by one laser wavelength. The bottom plot shows the differentiation of the upper trace. This illustrates that near resonance the cavity power changes very rapidly when the cavity length changes. However, for most tunings the cavity seems not sensitive at all.

\subsection{The Pound-Drever-Hall length sensing scheme}

This scheme for stabilising the frequency of a light field to the length of a cavity, or vice versa, is based on much older techniques for performing very similar actions with microwaves and microwave resonators. Drever and Hall have adapted such techniques for use in the optical regime [14] and today what is now called the Pound-Drever-Hall technique can be found in a great number of different types of optical setups. An example layout of this scheme is shown in Figure 34, in this case for generating a length (or frequency) signal of a two-mirror cavity. The laser is passed through an electro-optical modulator, which applies a periodic phase modulation at a fixed frequency. In many cases the modulation frequency is chosen such that it resides in the radio frequency band for which low-cost, low-noise electronic components are available. The phase modulated light is then injected into the cavity. However, from the frequency domain analysis introduced in Section 5, we know that in most cases not all the light can be injected into the cavity. Let's consider the example of an over-coupled cavity with the reflectivity of the end mirror $R_{2}<1$. Such a cavity would have a frequency response as shown in the top traces of Figure 26 (recall that the origin of the frequency axis refers to an arbitrarily chosen default frequency, which for this figure has been selected to be a resonance frequency of the cavity). If the cavity is held on resonance for the unmodulated carrier field, this field enters the cavity, gets resonantly enhanced and a substantial fraction is transmitted. If the frequency offset of the modulation sidebands is chosen such that it does not coincide with (or is near to) an integer multiple of the cavity's free spectral range, the modulation sidebands are mostly reflected by the cavity and will not be influenced as much by the resonance condition of the cavity as the carrier. The photodiode measuring the reflected light will see the optical beat between the carrier field and the modulation sidebands. This includes a component at the modulation frequency which is a measure of the phase difference between the carrier field and the sidebands (given the setup as described above). Any slight change of the 
cavity length would introduce a proportional change in the phase of the carrier field and no change in the sideband fields. Thus the photodiode signal can be used to measure the length changes of the cavity. One of the advantages of this method is the fact that the so-generated signal is bipolar with a zero crossing and steep slope exactly at the cavity's resonance, see Figure 35.

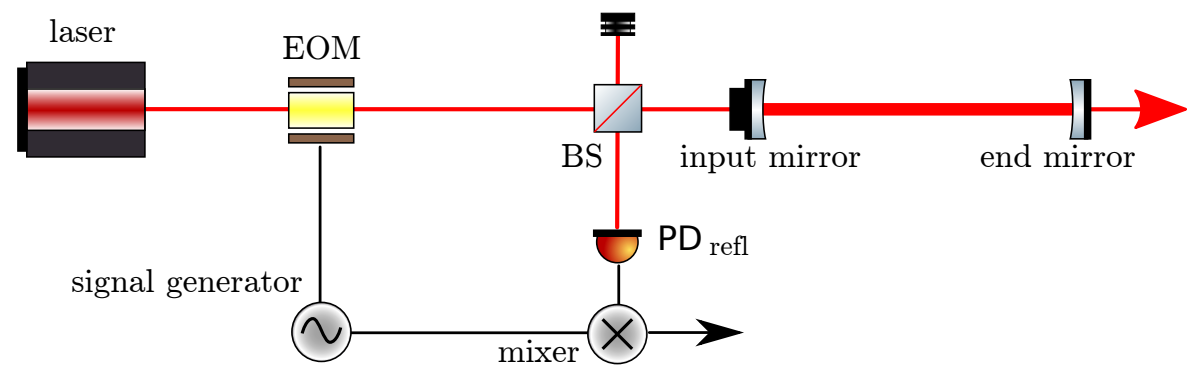

Figure 34: Typical setup for using the Pound-Drever-Hall scheme for length sensing and with a twomirror cavity: the laser beam is phase modulated with an electro-optical modulator (EOM). The modulation frequency is often in the radio frequency range. The photodiode signal in reflection is then electrically demodulated at the same frequency.

\subsection{Michelson length sensing}

Similarly to the two-mirror cavity, we can start to understand the length-sensing capabilities of the Michelson interferometer by looking at the output light power as a function of a mirror movement, as shown in Figure 29. The power changes as sine squared with the maximum slope at the point when the output power (in what we call the South port) is half the input power. The slope of the output power, which is the optical gain of the instrument for detecting a differential arm-length change $\Delta L$ with a photo detector in the South port can be written as

$$
\frac{d S}{d \Delta L}=\frac{2 \pi P_{0}}{\lambda} \sin \left(\frac{4 \pi}{\lambda} \Delta L\right)
$$

and is shown in Figure 36. The most notable difference of the optical gain of the Michelson interferometer with respect to the Fabry-Pérot interferometer (see Figure 33) is the wider, more smooth distribution of the gain. This is due to the fact that the cavity example is based on a highfinesse cavity in which the optical resonance effect is dominant. In a basic Michelson interferometer such resonance enhancement is not present.

However, the main difference is that the measurement is made differentially by comparing two lengths. This allows one to separate a larger number of possible noise contributions, for example noise in the laser light source, such as amplitude or frequency noise. This is why the main instrument for gravitational-wave measurements is a Michelson interferometer. However, the resonant enhancement of light power can be added to the Michelson, for example, by using FabryPérot cavities within the Michelson. This construction of new topologies by combining Michelson and Fabry-Pérot interferometers will be described in detail in a future version of this review.

The Michelson interferometer has two longitudinal degrees of freedom. These can be represented by the positions (along the optical axes) of the end mirrors. However, it is more efficient to use proper linear combinations of these and describe the Michelson interferometer length or position information by the common and differential arm length, as introduced in Equation (97):

$$
\begin{aligned}
& \bar{L}=\frac{L_{N}+L_{E}}{2} \\
& \Delta L=L_{N}-L_{E} .
\end{aligned}
$$

The Michelson interferometer is intrinsically insensitive to the common arm length $\bar{L}$.

Living Reviews in Relativity

http: //www. livingreviews.org/lrr-2010-1 

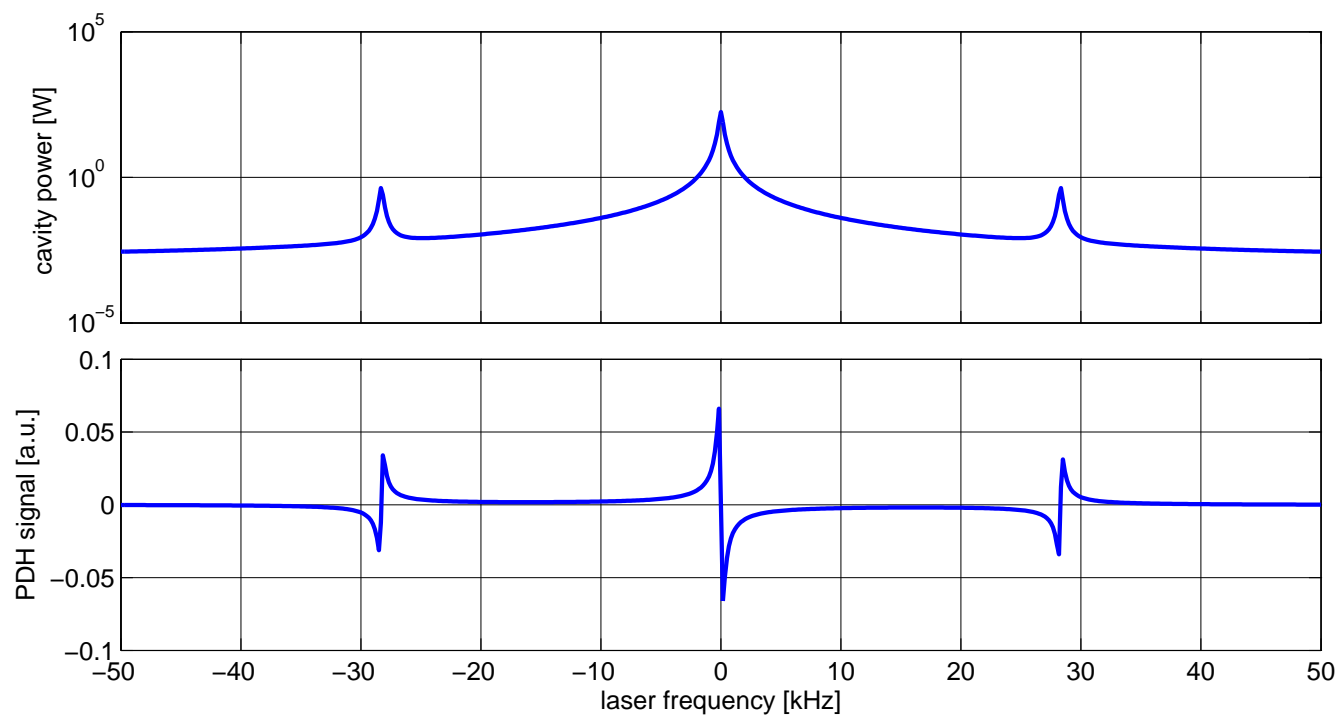

Figure 35: This figure shows an example of a Pound-Drever-Hall (PDH) signal of a two-mirror cavity. The plots refer to a setup in which the cavity mirrors are stationary and the frequency of the input laser is tuned linearly. The upper trace shows the light power circulating in the cavity. The three peaks correspond to the frequency tunings for which the carrier (main central peak) or the modulation sidebands (smaller side peaks) are resonant in the cavity. The lower trace shows the PDH signal for the same frequency tuning. Coincident with the peaks in the upper trace are bipolar structures in the lower trace. Each of the bipolar structures would be suitable as a length-sensing signal. In most cases the central structure is used, as experimentally it can be easily identified because its slope has a different sign compared to the sideband structures.
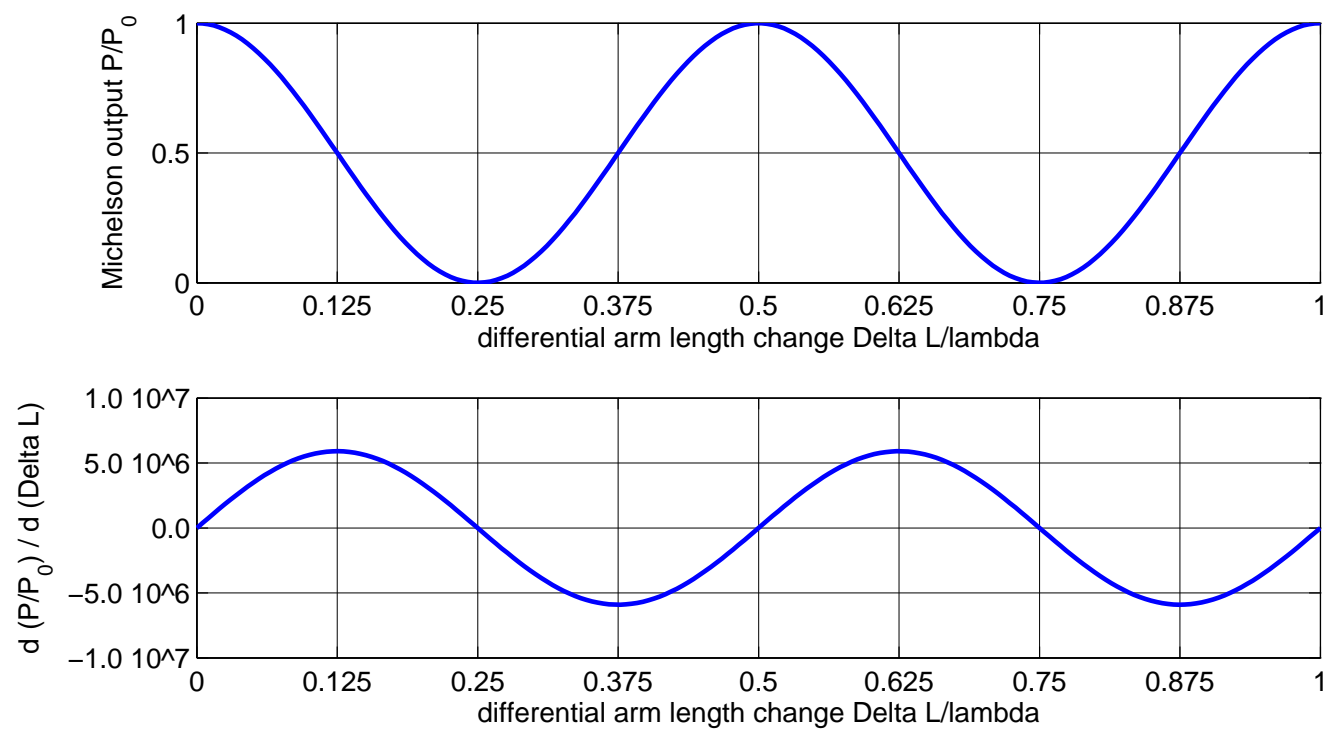

Figure 36: Power and slope of a Michelson interferometer. The upper plot shows the output power of a Michelson interferometer as detected in the South port (as already shown in Figure 29). The lower plot shows the optical gain of the instrument as given by the slope of the upper plot. 


\subsection{The Schnupp modulation scheme}

Similar to the Fabry-Pérot cavity, the Michelson interferometer is also often used to set an operating point where the optical gain of a direct light power detection is zero. This operating point, given by $\Delta L / \lambda=(2 N+1) \cdot 0.25$ with $N$ a non-negative integer, is called dark fringe. This operating point has several advantages, the most important being the low (ideally zero) light power on the diode. Highly efficient and low-noise photodiodes usually use a small detector area and thus are typically not able to detect large power levels. By using the dark fringe operating point, the Michelson interferometer can be used as a null instrument or null measurement, which generally is a good method to reduce systematic errors [49].

One approach to make use of the advantages of the dark fringe operating point is to use an operating point very close to the dark fringe at which the optical gain is not yet zero. In such a scenario a careful trade-off calculation can be done by computing the signal-to-noise with noises that must be suppressed, such as the laser amplitude noise. This type of operation is usually referred to as DC control or offset control and is very similar to the similarly-named mechanism used with Fabry-Pérot cavities.

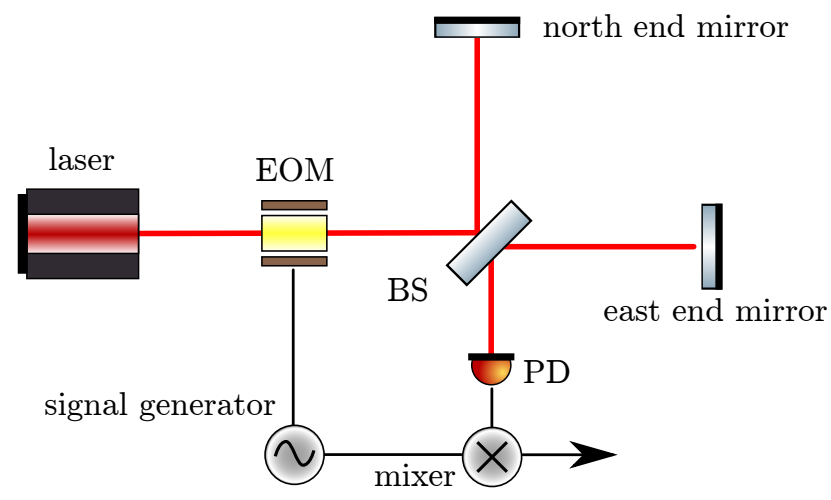

Figure 37: This length sensing scheme is often referred to as frontal or Schnupp modulation: an EOM is used to phase modulate the laser beam before entering the Michelson interferometer. The signal of the photodiode in the South port is then demodulated at the same frequency used for the modulation.

Another option is to employ phase modulated light, similar to the Pound-Drever-Hall scheme described in Section 6.3. The optical layout of such a scheme is depicted in Figure 37: an electrooptical modulator is used to apply a phase modulation at a fixed (usually RF type) frequency to the (monochromatic) laser light before it enters the interferometer. The photodiode signal from the interferometer output is then demodulated at the same frequency. This scheme allows one to operate the interferometer precisely on the dark fringe. The method originally proposed by Lise Schnupp is also sometimes referred to as frontal modulation.

The optical gain of a Michelson interferometer with Schnupp modulation is shown in Figure 39 in Section 6.6. 


\subsection{Finesse examples}

\subsubsection{Cavity power and slope}
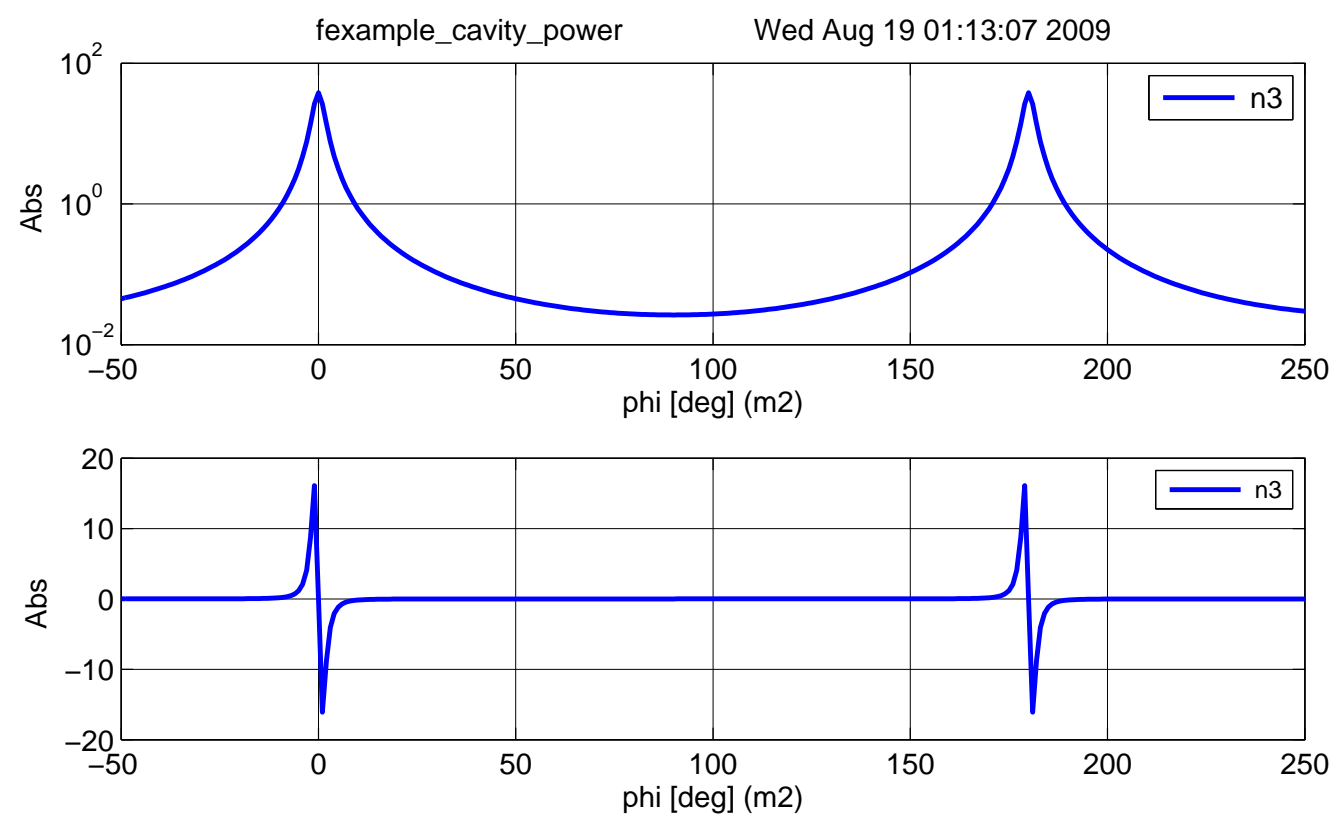

Figure 38: FinESSE example: Cavity power and slope.

Figure 33 shows a plot of the analytical functions describing the power inside a cavity and its differentiation by the cavity tuning. This example recreates the plot using a numerical model in FINESSE.

Finesse input file for 'Cavity power and slope'

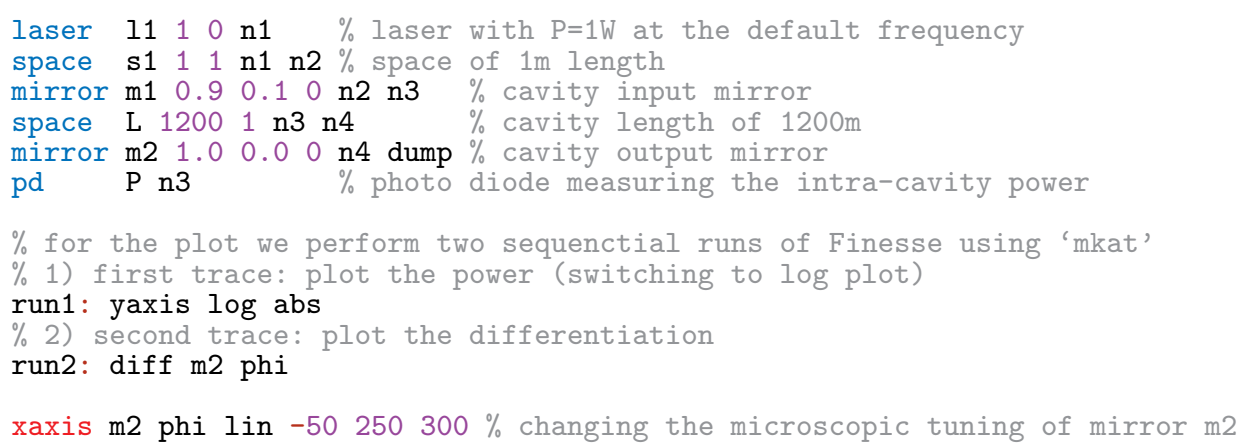

\subsubsection{Michelson with Schnupp modulation}

Figure 39 shows the demodulated photodiode signal of a Michelson interferometer with Schnupp modulation, as well as its differentiation, the latter being the optical gain of the system. Comparing this figure to Figure 36, it can be seen that with Schnupp modulation, the optical gain at the dark fringe operating points is maximised and a suitable error signal for these points is obtained. 


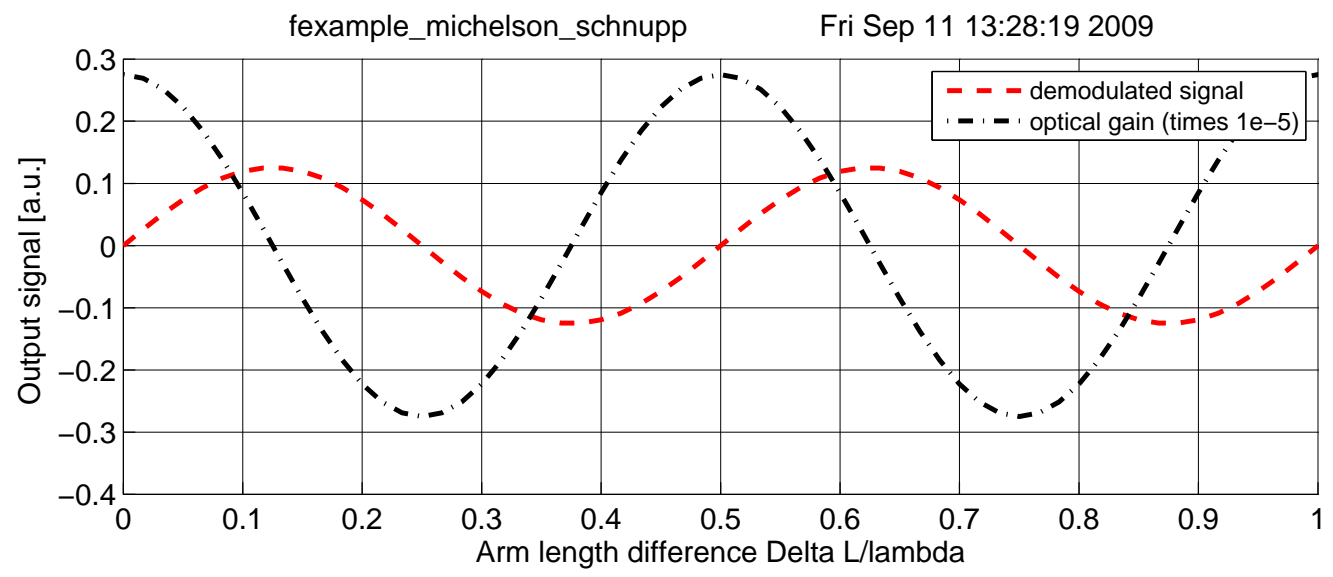

Figure 39: FinESSE example: Michelson with Schnupp modulation.

Finesse input file for 'Michelson with Schnupp modulation'

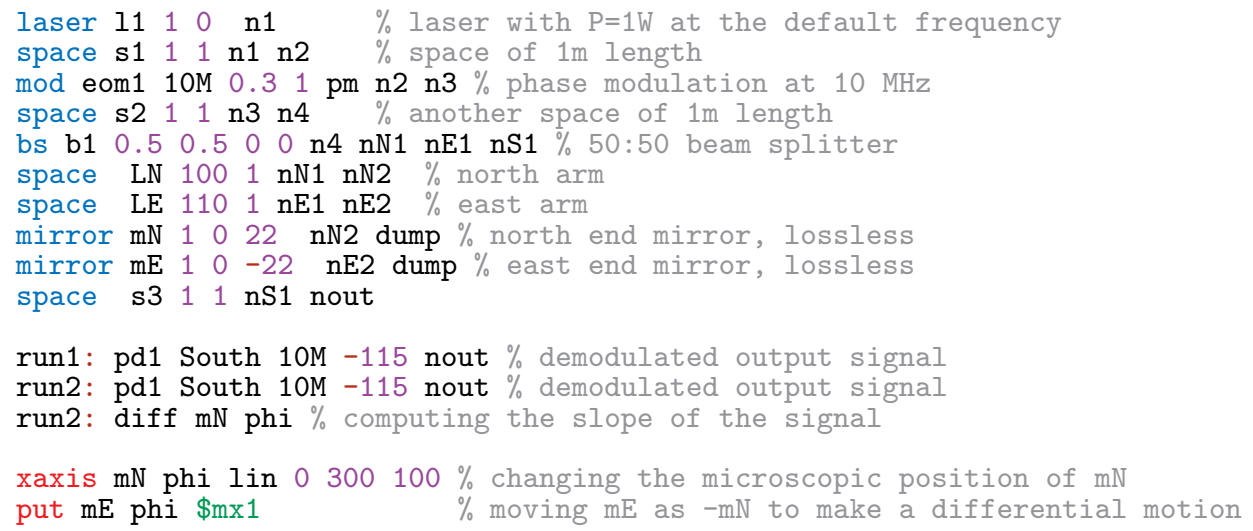




\section{Beam Shapes: Beyond the Plane Wave Approximation}

In previous sections we have introduced a notation for describing the on-axis properties of electric fields. Specifically, we have described the electric fields along an optical axis as functions of frequency (or time) and the location $z$. Models of optical systems may often use this approach for a basic analysis even though the respective experiments will always include fields with distinct offaxis beam shapes. A more detailed description of such optical systems needs to take the geometrical shape of the light field into account. One method of treating the transverse beam geometry is to describe the spatial properties as a sum of 'spatial components' or 'spatial modes' so that the electric field can be written as a sum of the different frequency components and of the different spatial modes. Of course, the concept of modes is directly related to the use of a sort of oscillator, in this case the optical cavity. Most of the work presented here is based on the research on laser resonators reviewed originally by Kogelnik and $\mathrm{Li}$ [35]. Siegman has written a very interesting historic review of the development of Gaussian optics $[52,51]$ and we use whenever possible the same notation as used in his textbook 'Lasers' [50].

This section introduces the use of Gaussian modes for describing the spatial properties along the transverse orthogonal $x$ and $y$ directions of an optical beam. We can write

$$
E(t, x, y, z)=\sum_{j} \sum_{n, m} a_{j n m} u_{n m}(x, y, z) \exp \left(\mathrm{i}\left(\omega_{j} t-k_{j} z\right)\right),
$$

with $u_{n m}$ as special functions describing the spatial properties of the beam and $a_{j n m}$ as complex amplitude factors $\left(\omega_{j}\right.$ is again the angular frequency and $\left.k_{j}=\omega_{j} / c\right)$. For simplicity we restrict the following description to a single frequency component at one moment in time $(t=0)$, so

$$
E(x, y, z)=\exp (-\mathrm{i} k z) \sum_{n, m} a_{n m} u_{n m}(x, y, z) .
$$

In general, different types of spatial modes $u_{n m}$ can be used in this context. Of particular interest are the Gaussian modes, which will be used throughout this document. Many lasers emit light that closely resembles a Gaussian beam: the light mainly propagates along one axis, is well collimated around that axis and the cross section of the intensity perpendicular to the optical axis shows a Gaussian distribution. The following sections provide the basic mathematical framework for using Gaussian modes for analysing optical systems.

\subsection{The paraxial wave equation}

Mathematically, Gaussian modes are solutions to the paraxial wave equation - a specific wave equation for electromagnetic fields. All electromagnetic waves are solutions to the general wave equation, which in vacuum can be given as:

$$
\Delta \vec{E}-\frac{1}{c^{2}} \ddot{\vec{E}}=0 .
$$

But laser light fields are special types of electromagnetic waves. For example, they are characterised by low diffraction. Hence, a laser beam will have a characteristic length $w$ describing the 'width' (the dimension of the field transverse to the main propagation axis), and a characteristic length $l$ defining some local length along the propagation over which the beam characteristics do not vary much. By definition, for what we call a beam $w$ is typically small and $l$ large in comparison, so that $w / l$ can be considered small. In fact, the paraxial wave equation (and its solutions) can be derived as the first-order terms of a series expansion of Equation (109) into orders of $w / l$ [37]. 
A simpler approach to the paraxial-wave equation goes as follows: A particular beam shape shall be described by a function $u(x, y, z)$ so that we can write the electric field as

$$
E(x, y, z)=u(x, y, z) \exp (-\mathrm{i} k z) .
$$

Substituting this into the standard wave equation yields a differential equation for $u$ :

$$
\left(\partial_{x}^{2}+\partial_{y}^{2}+\partial_{z}^{2}\right) u(x, y, z)-2 \mathrm{i} k \partial_{z} u(x, y, z)=0 .
$$

Now we put the fact that $u(x, y, z)$ should be slowly varying with $z$ in mathematical terms. The variation of $u(x, y, z)$ with $z$ should be small compared to its variation with $x$ or $y$. Also the second partial derivative in $z$ should be small. This can be expressed as

$$
\left|\partial_{z}^{2} u(x, y, z)\right| \ll\left|2 k \partial_{z} u(x, y, z)\right|,\left|\partial_{x}^{2} u(x, y, z)\right|,\left|\partial_{y}^{2} u(x, y, z)\right| .
$$

With this approximation, Equation (111) can be simplified to the paraxial wave equation,

$$
\left(\partial_{x}^{2}+\partial_{y}^{2}\right) u(x, y, z)-2 \mathrm{i} k \partial_{z} u(x, y, z)=0 .
$$

Any field $u$ that solves this equation represents a paraxial beam shape when used in the form given in Equation (110).

\subsection{Transverse electromagnetic modes}

In general, any solution $u(x, y, z)$ of the paraxial wave equation, Equation (113), can be employed to represent the transverse properties of a scalar electric field representing a beam-like electromagnetic wave. Especially useful in this respect are special families or sets of functions that are solutions of the paraxial wave equation. When such a set of functions is complete and countable, it's called a set of transverse electromagnetic modes (TEM). For instance, the set of HermiteGauss modes are exact solutions of the paraxial wave equation. These modes are represented by an infinite, countable and complete set of functions. The term complete means they can be understood as a base system of the function space defined by all solutions of the paraxial wave equation. In other words, we can describe any solution of the paraxial wave equation $u^{\prime}$ by a linear superposition of Hermite-Gauss modes:

$$
u^{\prime}(x, y, z)=\sum_{n, m} a_{j n m} u_{n m}(x, y, z)
$$

which in turn allows us to describe any laser beam using a sum of these modes:

$$
E(t, x, y, z)=\sum_{j} \sum_{n, m} a_{j n m} u_{n m}(x, y, z) \exp \left(\mathrm{i}\left(\omega_{j} t-k_{j} z\right)\right) .
$$

The Hermite-Gauss modes as given in this document (see Section 7.5) are orthonormal so that

$$
\iint d x d y u_{n m} u_{n^{\prime} m^{\prime}}^{*}=\delta_{n n^{\prime}} \delta_{m m^{\prime}}=\left\{\begin{array}{ll}
1 & \text { if } n=n^{\prime} \\
0 & \text { otherwise }
\end{array} \text { and } m=m^{\prime}\right\} .
$$

This means that, in the function space defined by the paraxial wave equation, the Hermite-Gauss functions can be understood as a complete set of unit-length basis vectors. This fact can be utilised for the computation of coupling factors. Furthermore, the power of a beam, as given by Equation (108), being detected on a single-element photodetector (provided that the area of the detector is large with respect to the beam) can be computed as

$$
E E^{*}=\sum_{n, m} a_{n m} a_{n m}^{*}
$$


or for a beam with several frequency components (compare with Equation (76)) as

$$
E E^{*}=\sum_{n, m} \sum_{i} \sum_{j} a_{i n m} a_{j n m}^{*} \quad \text { with } \quad\left\{i, j \mid i, j \in\{0, \ldots, N\} \wedge \omega_{i}=\omega_{j}\right\} .
$$

\subsection{Properties of Gaussian beams}

The basic or 'lowest-order' Hermite-Gauss mode is equivalent to what is usually called a Gaussian beam and is given by

$$
u(x, y, z)=\sqrt{\frac{2}{\pi}} \frac{1}{w(z)} \exp (\mathrm{i} \Psi(z)) \exp \left(-\mathrm{i} k \frac{x^{2}+y^{2}}{2 R_{C}(z)}-\frac{x^{2}+y^{2}}{w^{2}(z)}\right) .
$$

The parameters of this equation are explained in detail below. The shape of a Gaussian beam is quite simple: the beam has a circular cross section, and the radial intensity profile of a beam with total power $P$ is given by

$$
I(r)=\frac{2 P}{\pi w^{2}(z)} \exp \left(-2 r^{2} / w^{2}\right),
$$

with $w$ the spot size, defined as the radius at which the intensity is $1 / e^{2}$ times the maximum intensity $I(0)$. This is a Gaussian distribution, see Figure 40, hence the name Gaussian beam.

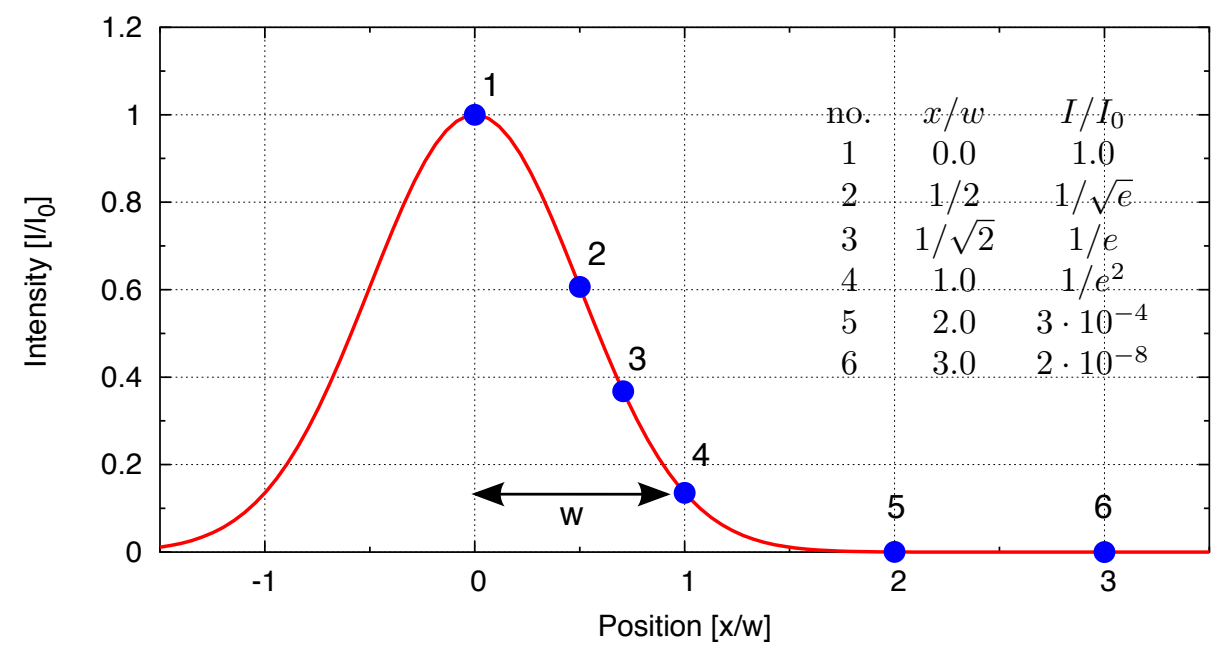

Figure 40: One dimensional cross-section of a Gaussian beam. The width of the beam is given by the radius $w$ at which the intensity is $1 / e^{2}$ of the maximum intensity.

Figure 41 shows a different cross section through a Gaussian beam: it plots the beam size as a function of the position on the optical axis.

Such a beam profile (for a beam with a given wavelength $\lambda$ ) can be completely determined by two parameters: the size of the minimum spot size $w_{0}$ (called beam waist) and the position $z_{0}$ of the beam waist along the $z$-axis.

To characterise a Gaussian beam, some useful parameters can be derived from $w_{0}$ and $z_{0}$. A Gaussian beam can be divided into two different sections along the $z$-axis: a near field - a region around the beam waist, and a far field - far away from the waist. The length of the near-field region is approximately given by the Rayleigh range $z_{\mathrm{R}}$. The Rayleigh range and the spot size are related by

$$
z_{\mathrm{R}}=\frac{\pi w_{0}^{2}}{\lambda} .
$$




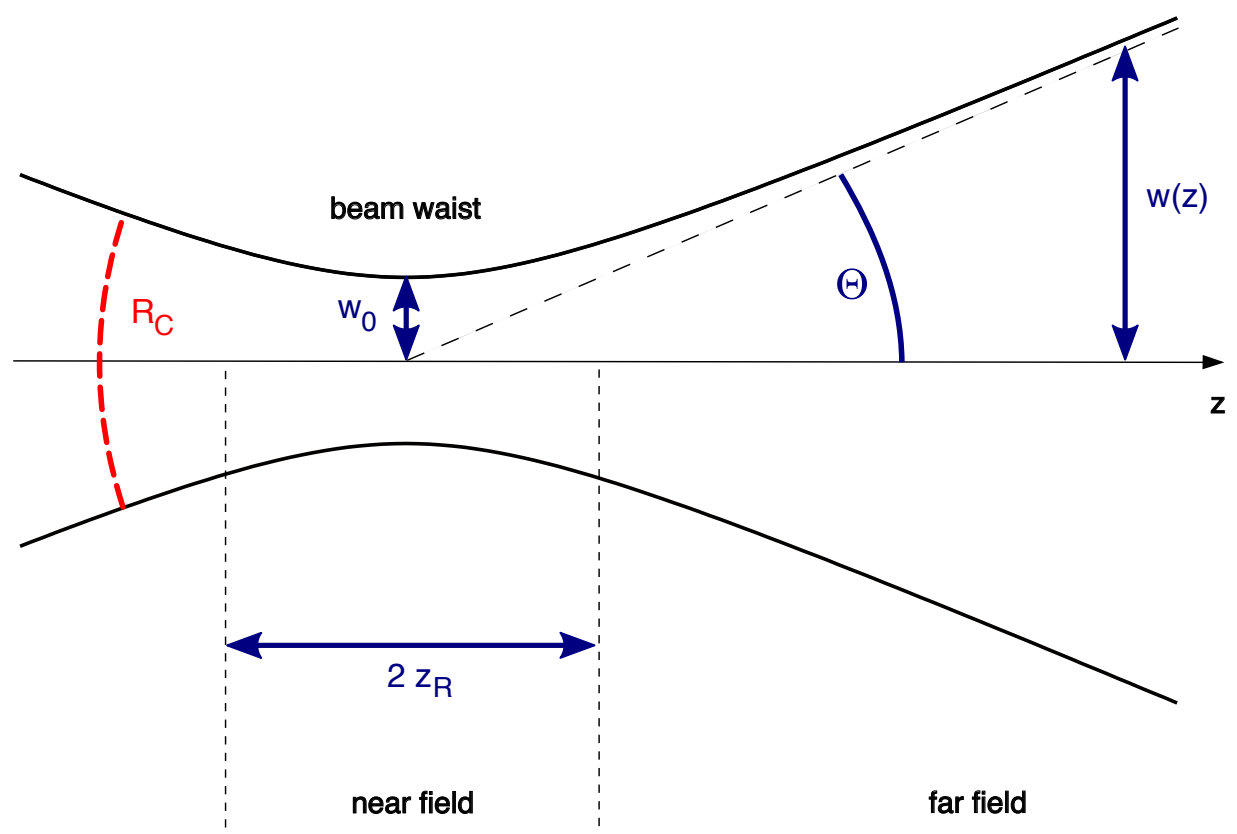

Figure 41: Gaussian beam profile along z: this cross section along the $x$ - $z$-plane illustrates how the beam size $w(z)$ of the Gaussian beam changes along the optical axis. The position of minimum beam size $w_{0}$ is called beam waist. See text for a description of the parameters $\Theta, z_{R}$ and $R_{c}$.

With the Rayleigh range and the location of the beam waist, we can usefully write

$$
w(z)=w_{0} \sqrt{1+\left(\frac{z-z_{0}}{z_{\mathrm{R}}}\right)^{2}}
$$

This equation gives the size of the beam along the $z$-axis. In the far-field regime $\left(z \gg z_{\mathrm{R}}, z_{0}\right)$, it can be approximated by a linear equation, when

$$
w(z) \approx w_{0} \frac{z}{z_{\mathrm{R}}}=\frac{z \lambda}{\pi w_{0}} .
$$

The angle $\Theta$ between the $z$-axis and $w(z)$ in the far field is called the diffraction angle ${ }^{6}$ and is defined by

$$
\Theta=\arctan \left(\frac{w_{0}}{z_{\mathrm{R}}}\right)=\arctan \left(\frac{\lambda}{\pi w_{0}}\right) \approx \frac{w_{0}}{z_{\mathrm{R}}} .
$$

Another useful parameter is the radius of curvature of the wavefront at a given point $z$. The radius of curvature describes the curvature of the 'phase front' of the electromagnetic wave - a surface across the beam with equal phase - intersecting the optical axis at the position $z$. We obtain the radius of curvature as a function of $z$ :

$$
R_{C}(z)=z-z_{0}+\frac{z_{\mathrm{R}}^{2}}{z-z_{0}}
$$

\footnotetext{
${ }^{6}$ Also known as the far-field angle or the divergence of the beam.
} 
We also find:

$$
\begin{aligned}
& R_{C} \approx \infty, \quad z-z_{0} \ll z_{\mathrm{R}} \quad \text { (beam waist) } \\
& R_{C} \approx z, \quad z \gg z_{\mathrm{R}}, z_{0} \quad \text { (far field) } \\
& R_{C}=2 z_{\mathrm{R}}, z-z_{0}=z_{\mathrm{R}} \quad \text { (maximum curvature). }
\end{aligned}
$$

\subsection{Astigmatic beams: the tangential and sagittal plane}

If the interferometer is confined to a plane (here the $x$ - $z$ plane), it is convenient to use projections of the three-dimensional description into two planes [46]: the tangential plane, defined as the $x-z$ plane and the sagittal plane as given by $y$ and $z$.

The beam parameters can then be split into two respective parameters: $z_{0, s}, w_{0, s}$ for the sagittal plane and $z_{0, t}$ and $w_{0, t}$ for the tangential plane so that the Hermite-Gauss modes can be written as

$$
u_{n m}(x, y)=u_{n}\left(x, z_{0, t}, w_{0, t}\right) u_{m}\left(y, z_{0, s}, w_{0, s}\right) .
$$

Beams with different beam waist parameters for the sagittal and tangential plane are astigmatic.

Remember that these Hermite-Gauss modes form a base system. This means one can use the separation into sagittal and tangential planes even if the actual optical system does not show this special type of symmetry. This separation is very useful in simplifying the mathematics. In the following, the term beam parameter generally refers to a simple case where $w_{0, x}=w_{0, y}$ and $z_{0, x}=z_{0, y}$ but all the results can also be applied directly to a pair of parameters.

\subsection{Higher-order Hermite-Gauss modes}

The complete set of Hermite-Gauss modes is given by an infinite discrete set of modes $u_{\mathrm{nm}}(x, y, z)$ with the indices $\mathrm{n}$ and $\mathrm{m}$ as mode numbers. The sum $\mathrm{n}+\mathrm{m}$ is called the order of the mode. The term higher-order modes usually refers to modes with an order $n+m>0$. The general expression for Hermite-Gauss modes can be given as [35]

$$
u_{\mathrm{nm}}(x, y, z)=u_{\mathrm{n}}(x, z) u_{\mathrm{m}}(y, z)
$$

with

$$
\begin{aligned}
u_{\mathrm{n}}(x, z)= & \left(\frac{2}{\pi}\right)^{1 / 4}\left(\frac{\exp (\mathrm{i}(2 n+1) \Psi(z))}{2^{n} n ! w(z)}\right)^{1 / 2} \times \\
& H_{n}\left(\frac{\sqrt{2} x}{w(z)}\right) \exp \left(-\mathrm{i} \frac{k x^{2}}{2 R_{C}(z)}-\frac{x^{2}}{w^{2}(z)}\right),
\end{aligned}
$$

and $H_{n}(x)$ the Hermite polynomials of order n. The first Hermite polynomials, without normalisation, can be written

$$
\begin{array}{ll}
H_{0}(x) & =1 \quad H_{1}(x)=2 x \\
H_{2}(x) & =4 x^{2}-2 H_{3}(x)=8 x^{3}-12 x .
\end{array}
$$

Further orders can be computed recursively since

$$
H_{n+1}(x)=2 x H_{n}(x)-2 n H_{n-1}(x) .
$$

For both transverse directions we can also rewrite the above to

$$
\begin{array}{r}
u_{\mathrm{nm}}(x, y, z)=\left(2^{n+m-1} n ! m ! \pi\right)^{-1 / 2} \frac{1}{w(z)} \exp (\mathrm{i}(n+m+1) \Psi(z)) \times \\
H_{n}\left(\frac{\sqrt{2} x}{w(z)}\right) H_{m}\left(\frac{\sqrt{2} y}{w(z)}\right) \exp \left(-\mathrm{i} \frac{k\left(x^{2}+y^{2}\right)}{2 R_{C}(z)}-\frac{x^{2}+y^{2}}{w^{2}(z)}\right) .
\end{array}
$$

The latter form has the advantage of clearly showing the extra phase shift along the $z$-axis of $(n+m+1) \Psi(z)$, called the Gouy phase; see Section 7.8. 


\subsection{The Gaussian beam parameter}

For a more compact description of the interaction of Gaussian modes with optical components we will make use of the Gaussian beam parameter $q$ [34]. The beam parameter is a complex quantity defined as

$$
\frac{1}{q(z)}=\frac{1}{R_{C}(z)}-\mathrm{i} \frac{\lambda}{\pi w^{2}(z)}
$$

It can also be written as

$$
q(z)=\mathrm{i} z_{\mathrm{R}}+z-z_{0}=q_{0}+z-z_{0} \quad \text { and } \quad q_{0}=\mathrm{i} z_{\mathrm{R}} .
$$

Using this parameter, Equation (119) can be rewritten as

$$
u(x, y, z)=\frac{1}{q(z)} \exp \left(-\mathrm{i} k \frac{x^{2}+y^{2}}{2 q(z)}\right) .
$$

Other parameters, like the beam size and radius of curvature, can also be written in terms of the beam parameter $q$ :

$$
\begin{gathered}
w^{2}(z)=\frac{\lambda}{\pi} \frac{|q|^{2}}{\Im\{q\}}, \\
w_{0}^{2}=\frac{\Im\{q\} \lambda}{\pi}, \\
z_{\mathrm{R}}=\Im\{q\}
\end{gathered}
$$

and

$$
R_{C}(z)=\frac{|q|^{2}}{\Re\{q\}} .
$$

The Hermite-Gauss modes can also be written using the Gaussian beam parameter as ${ }^{7}$

$$
\begin{aligned}
& u_{\mathrm{nm}}(x, y, z)=u_{\mathrm{n}}(x, z) u_{\mathrm{m}}(y, z) \quad \text { with } \\
& u_{\mathrm{n}}(x, z)=\left(\frac{2}{\pi}\right)^{1 / 4}\left(\frac{1}{2^{n} n ! w_{0}}\right)^{1 / 2}\left(\frac{q_{0}}{q(z)}\right)^{1 / 2}\left(\frac{q_{0} q^{*}(z)}{q_{0}^{*} q(z)}\right)^{n / 2} H_{n}\left(\frac{\sqrt{2} x}{w(z)}\right) \exp \left(-\mathrm{i} \frac{k x^{2}}{2 q(z)}\right) .
\end{aligned}
$$

\footnotetext{
${ }^{7}$ Please note that this formula from [50] is very compact. Since the parameter $q$ is a complex number, the expression contains at least two complex square roots. The complex square root requires a different algebra than the standard square root for real numbers. Especially the third and fourth factors can not be simplified in any obvious way: $\left(\frac{q_{0}}{q(z)}\right)^{1 / 2}\left(\frac{q_{0} q^{*}(z)}{q_{0}^{*} q(z)}\right)^{n / 2} \neq\left(\frac{q_{0}^{n+1} q^{* n}(z)}{q^{n+1}(z) q_{0}^{* n}}\right)^{1 / 2}$ !
} 


\subsection{Properties of higher-order Hermite-Gauss modes}

Some of the properties of Hermite-Gauss modes can easily be described using cross sections of the field intensity or field amplitude. Figure 42 shows such cross sections, i.e., the intensity in the $x$ - $y$ plane, for a number of higher-order modes. This shows a $x-y$ symmetry for mode indices $n$ and $m$. We can also see how the size of the intensity distribution increases with the mode index, while the peak intensity decreases.

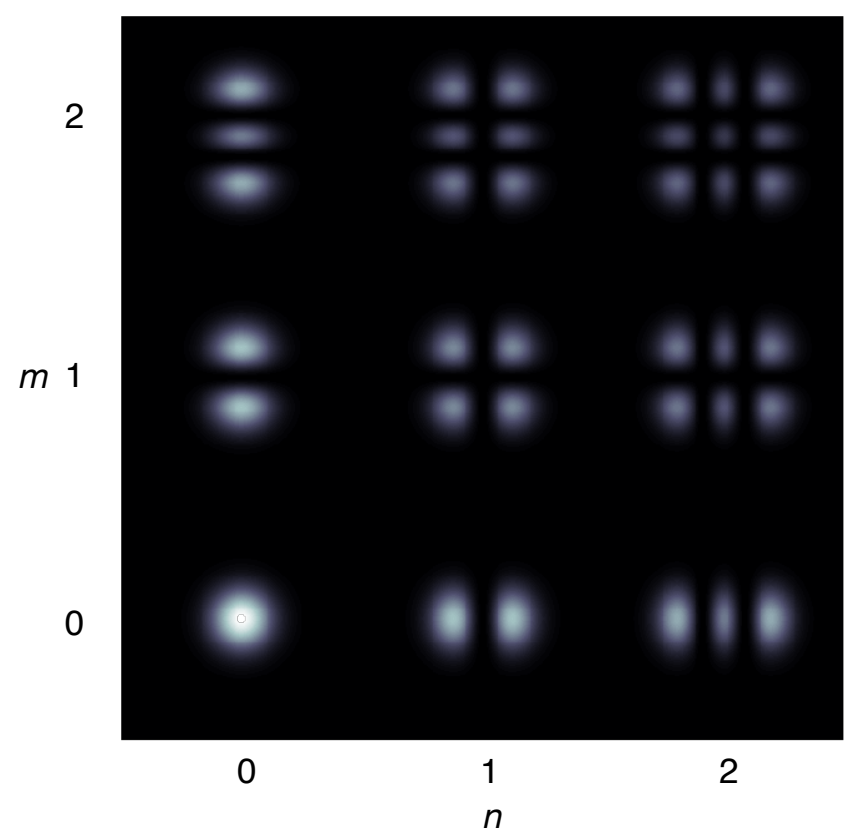

Figure 42: This plot shows the intensity distribution of Hermite-Gauss modes $u_{n m}$. One can see that the intensity distribution becomes wider for larger mode indices and the peak intensity decreases. The mode index defines the number of dark stripes in the respective direction.

Similarly, Figure 44 shows the amplitude and phase distribution of several higher-order HermiteGauss modes. Some further features of Hermite-Gauss modes:

- The size of the intensity profile of any sum of Hermite-Gauss modes depends on $z$ while its shape remains constant over propagation along the optical axis.

- The phase distribution of Hermite-Gauss modes shows the curvature (or radius of curvature) of the beam. The curvature depends on $z$ but is equal for all higher-order modes.

Note that these are special features of Gaussian beams and not generally true for arbitrary beam shapes. Figure 43, for example, shows the amplitude and phase distribution of a triangular beam at the point where it is (mathematically) created and after a $10 \mathrm{~m}$ propagation. Neither the shape is preserved nor does it show a spherical phase distribution. 

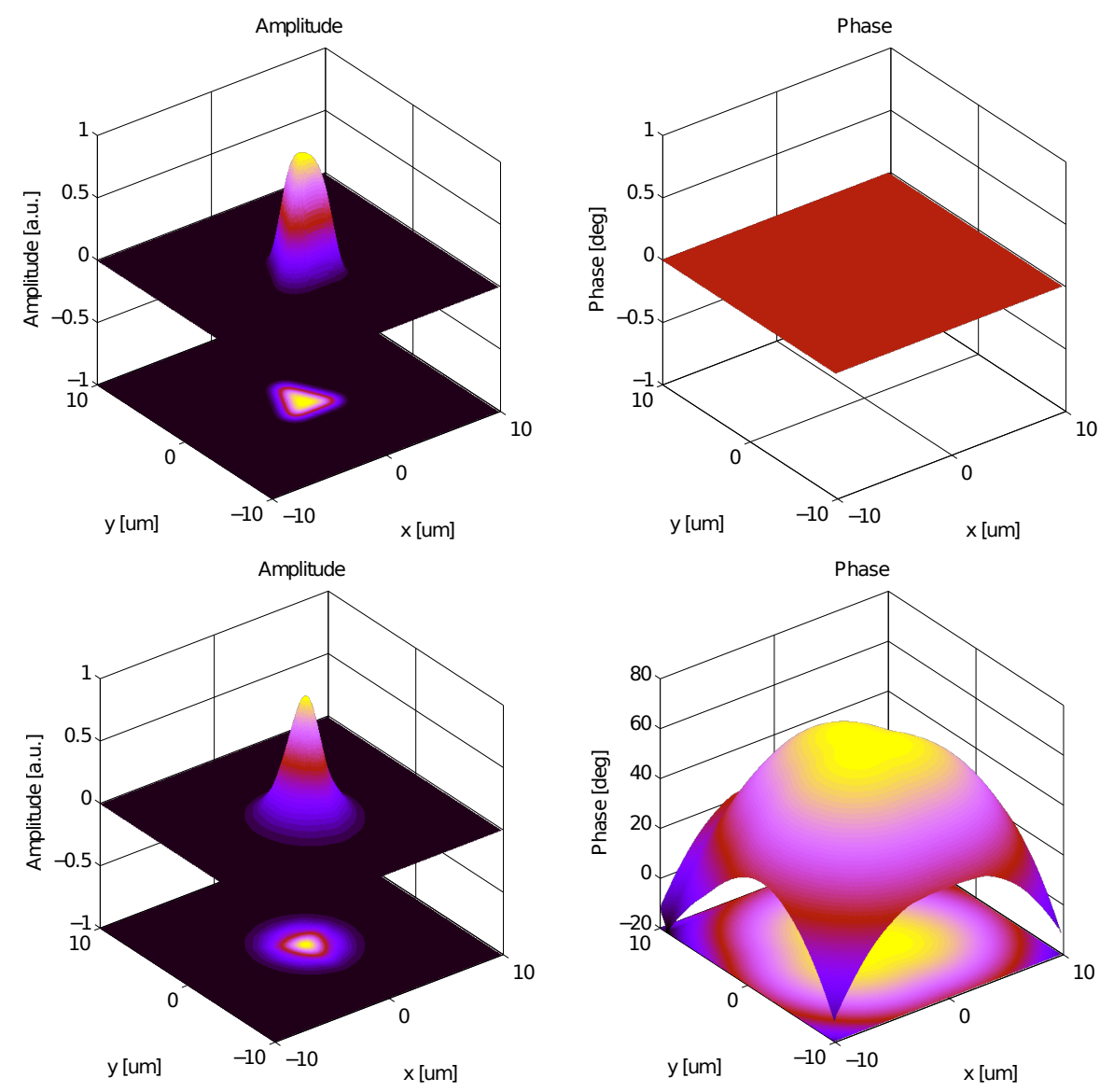

Figure 43: These top plots show a triangular beam shape and phase distribution and the bottom plots the diffraction pattern of this beam after a propagation of $z=5 \mathrm{~m}$. It can be seen that the shape of the triangular beam is not conserved and that the phase front is not spherical. 

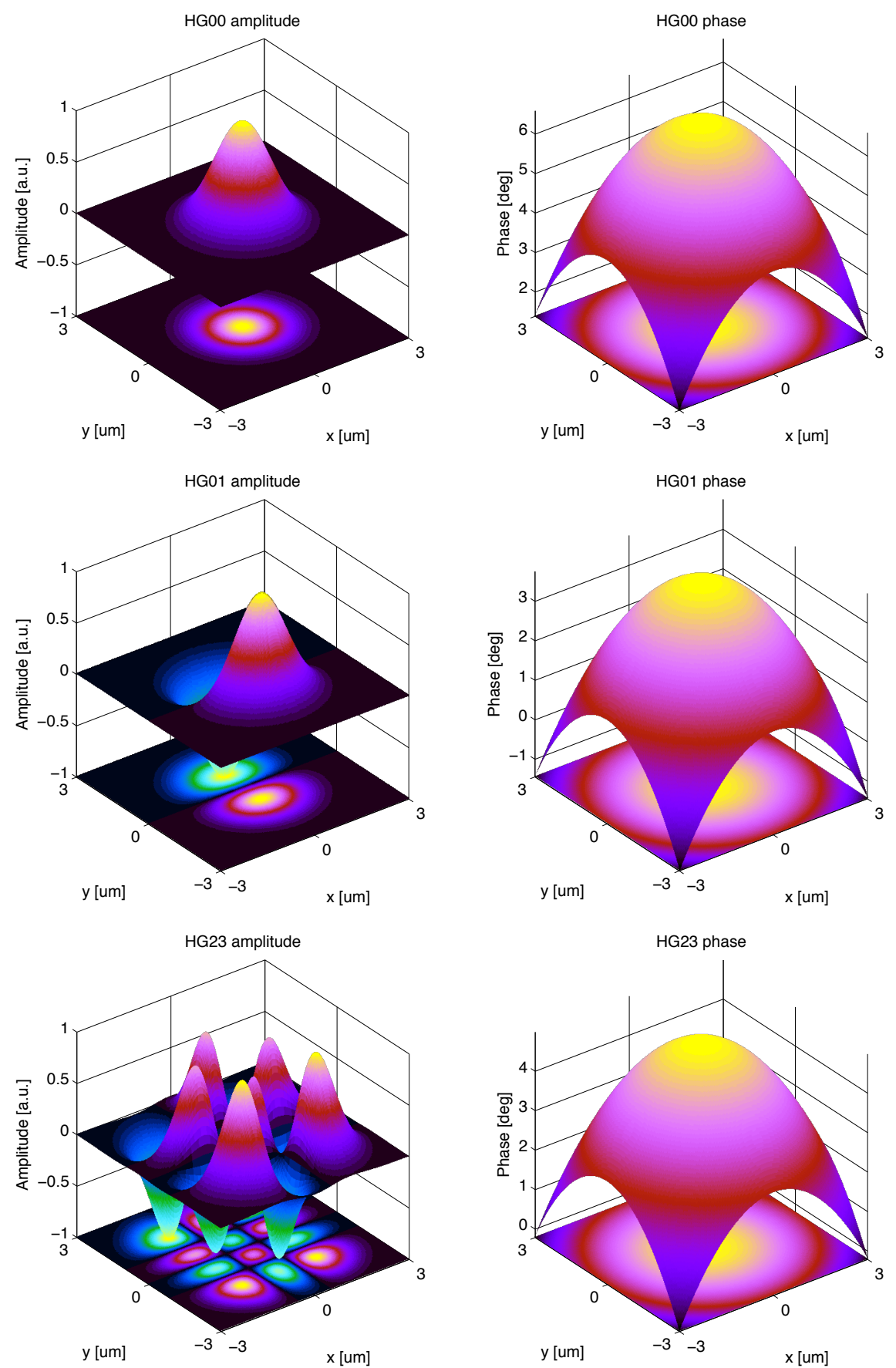

Figure 44: These plots show the amplitude distribution and wave front (phase distribution) of HermiteGaussian modes $u_{n m}$ (labeled as HGnm in the plot). All plots refer to a beam with $\lambda=1 \mu \mathrm{m}, w=1 \mathrm{~mm}$ and distance to waist $z=1 \mathrm{~m}$. The mode index (in one direction) defines the number of zero crossings (along that axis) in the amplitude distribution. One can also see that the phase distribution is the same spherical distribution, regardless of the mode indices. 


\subsection{Gouy phase}

The equation for Hermite-Gauss modes shows an extra longitudinal phase lag. This Gouy phase [8, 26, 25] describes the fact that, compared to a plane wave, the Hermite-Gauss modes have a slightly slower phase velocity, especially close to the waist. The Gouy phase can be written as

$$
\Psi(z)=\arctan \left(\frac{z-z_{0}}{z_{\mathrm{R}}}\right)
$$

or, using the Gaussian beam parameter,

$$
\Psi(z)=\arctan \left(\frac{\Re\{q\}}{\Im\{q\}}\right) .
$$

Compared to a plane wave, the phase lag $\varphi$ of a Hermite-Gauss mode is

$$
\varphi=(n+m+1) \Psi(z) .
$$

With an astigmatic beam, i.e., different beam parameters in the tangential and sagittal planes, this becomes

$$
\varphi=\left(n+\frac{1}{2}\right) \Psi_{t}(z)+\left(m+\frac{1}{2}\right) \Psi_{s}(z),
$$

with

$$
\Psi_{t}(z)=\arctan \left(\frac{\Re\left\{q_{t}\right\}}{\Im\left\{q_{t}\right\}}\right),
$$

as the Gouy phase in the tangential plane (and $\Psi_{s}$ is similarly defined in the sagittal plane).

\subsection{Laguerre-Gauss modes}

Laguerre-Gauss modes are another complete set of functions, which solve the paraxial wave equation. They are defined in cylindrical coordinates and can have advantages over Hermite-Gauss modes in the presence of cylindrical symmetry. More recently, Laguerre-Gauss modes are being investigated in a different context: using a pure higher-order Laguerre-Gauss mode instead of the fundamental Gaussian beam can significantly reduce the impact of mirror thermal noise on the sensitivity of gravitational wave detectors [54, 12]. Laguerre-Gauss modes are commonly given as $[50]$

$$
\begin{aligned}
u_{p, l}(r, \phi, z) & =\frac{1}{w(z)} \sqrt{\frac{2 p !}{\pi(|l|+p) !}} \exp (\mathrm{i}(2 p+|l|+1) \Psi(z)) \\
& \times\left(\frac{\sqrt{2} r}{w(z)}\right)^{|l|} L_{p}^{(l)}\left(\frac{2 r^{2}}{w(z)^{2}}\right) \exp \left(-\mathrm{i} k \frac{r^{2}}{2 q(z)}+\mathrm{i} l \phi\right),
\end{aligned}
$$

with $r, \phi$ and $z$ as the cylindrical coordinates around the optical axis. The letter $p$ is the radial mode index, $l$ the azimuthal mode index ${ }^{8}$ and $L_{p}^{(l)}(x)$ are the associated Laguerre polynomials:

$$
L_{p}^{(l)}(x)=\frac{1}{p !} \sum_{j=0}^{p} \frac{p !}{j !}\left(\begin{array}{c}
l+p \\
p-j
\end{array}\right)(-x)^{j} .
$$

All other parameters $(w(z), q(z), \ldots)$ are defined as above for the Hermite-Gauss modes.

The dependence of the Laguerre modes on $\phi$ as given in Equation (146) results in a spiraling phase front, while the intensity pattern will always show unbroken concentric rings; see Figure 45. These modes are also called helical Laguerre-Gauss modes because of the their special phase structure.

${ }^{8}[50]$ states that the indices must obey the following relations: $0 \leq|l| \leq p$. However, that is not the case. 

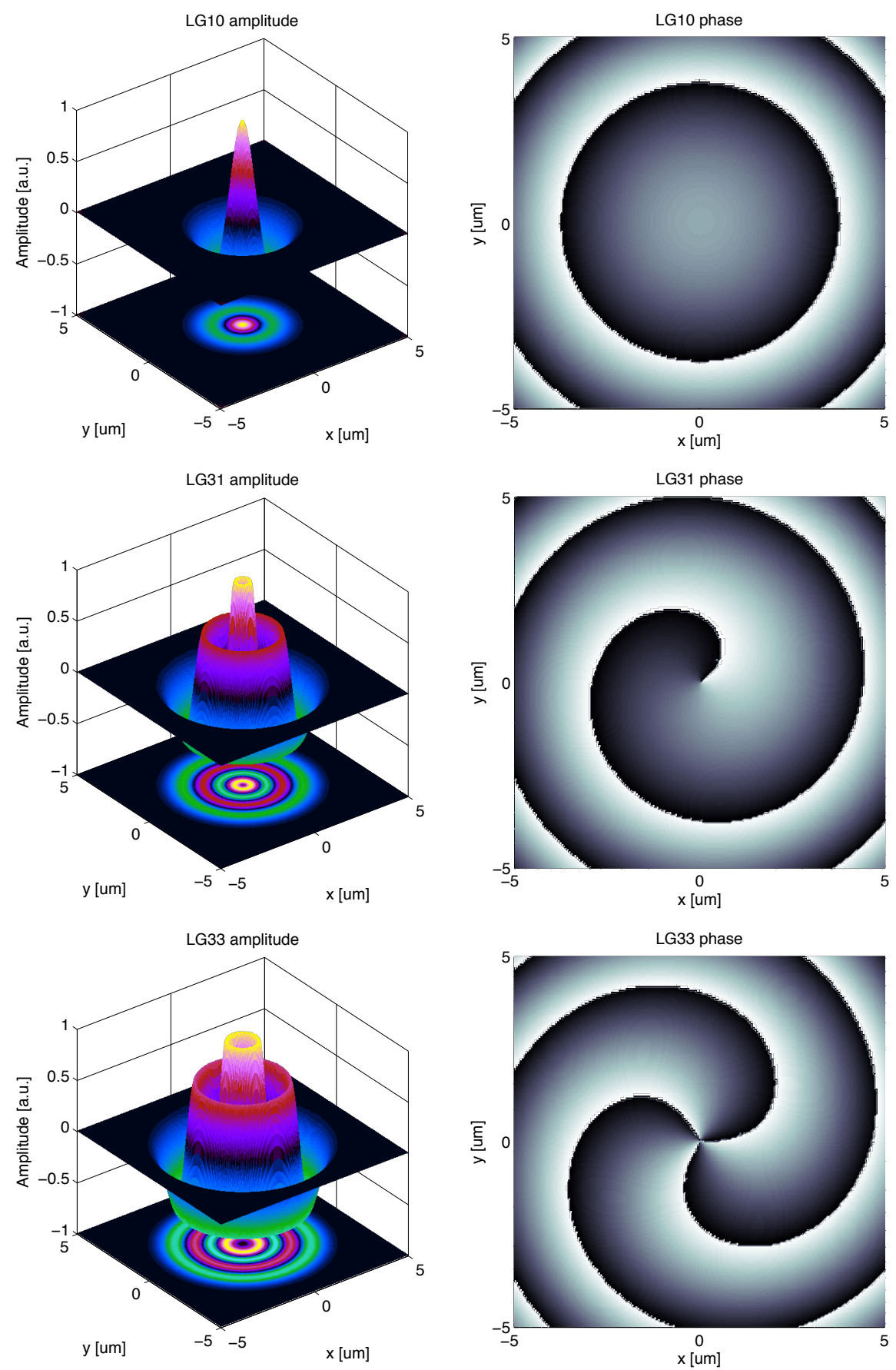

Figure 45: These plots show the amplitude distribution and wave front (phase distribution) of helical Laguerre-Gauss modes $u_{p l}$. All plots refer to a beam with $\lambda=1 \mu \mathrm{m}, w=1 \mathrm{~mm}$ and distance to waist $z=1 \mathrm{~m}$. 
The reader might be more familiar with a slightly different type of Laguerre modes (compare Figure 46 and Figure 47) that features dark radial lines as well as dark concentric rings. Mathematically, these can be described simply by replacing the phase factor $\exp (\mathrm{i} l \phi)$ in Equation (146) by a sine or cosine function. For example, an alternative set of Laguerre-Gauss modes is given by $[55]$

$$
\begin{aligned}
u_{p, l}^{\text {alt }}(r, \phi, z) & =\frac{2}{w(z)} \sqrt{\frac{p !}{\left(1+\delta_{0 l} \pi(|l|+p) !\right.}} \exp (\mathrm{i}(2 p+|l|+1) \Psi(z)) \\
& \times\left(\frac{\sqrt{2} r}{w(z)}\right)^{|l|} L_{p}^{(l)}\left(\frac{2 r^{2}}{w(z)^{2}}\right) \exp \left(-\mathrm{i} k \frac{r^{2}}{2 q(z)}\right) \cos (l \phi) .
\end{aligned}
$$

This type of mode has a spherical phase front, just as the Hermite-Gauss modes. We will refer to this set as sinusoidal Laguerre-Gauss modes throughout this document.

For the purposes of simulation it can be sometimes useful to decompose Laguerre-Gauss modes into Hermite-Gauss modes. The mathematical conversion for helical modes is given as [7, 1]

$$
u_{n, m}^{L G}(x, y, z)=\sum_{k=0}^{N} \mathrm{i}^{k} b(n, m, k) u_{N-k, k}^{H G}(x, y, z),
$$

with real coefficients

$$
b(n, m, k)=\sqrt{\frac{(N-k) ! k !}{2^{N} n ! m !}} \frac{1}{k !}\left(\partial_{t}\right)^{k}\left[(1-t)^{n}(1+t)^{m}\right]_{t=0},
$$

if $N=n+m$. This relates to the common definition of Laguerre modes as $u_{p l}$ as follows: $p=$ $\min (n, m)$ and $l=n-m$. The coefficients $b(n, m, k)$ can be computed numerically by using Jacobi polynomials. Jacobi polynomials can be written in various forms:

$$
P_{n}^{\alpha, \beta}(x)=\frac{(-1)^{n}}{2^{n} n !}(1-x)^{-\alpha}(1+x)^{-\beta}\left(\partial_{x}\right)^{n}(1-x)^{\alpha+n}(1+x)^{\beta+n}
$$

or

$$
P_{n}^{\alpha, \beta}(x)=\frac{1}{2^{n}} \sum_{j=0}^{n}\left(\begin{array}{c}
n+\alpha \\
j
\end{array}\right)\left(\begin{array}{c}
n+\beta \\
n-j
\end{array}\right)(x-1)^{n-j}(x+1)^{j},
$$

which leads to

$$
b(n, m, k)=\sqrt{\frac{(N-k) ! k !}{2^{N} n ! m !}}(-2)^{k} P_{k}^{n-k, m-k}(0) .
$$

\subsection{Tracing a Gaussian beam through an optical system}

Whenever Gauss modes are used to analyse an optical system, the Gaussian beam parameters (or equivalent waist sizes and locations) must be defined for each location at which field amplitudes are to be computed (or at which coupling equations are to be defined). In our experience the quality of a computation or simulation and the correctness of the results depend critically on the choice of these beam parameters. One might argue that the choice of a basis should not alter the result. This is correct, but there is a practical limitation: the number of modes having non-negligible power might become very large if the beam parameters are not optimised, so that in practice a good set of beam parameters is usually required.

In general, the Gaussian beam parameter of a mode is changed at every optical surface in a welldefined way (see Section 7.11). Thus, a possible method of finding reasonable beam parameters for every location in the interferometer is to first set only some specific beam parameters at selected locations and then to derive the remaining beam parameters from these initial ones: usually it is sensible to assume that the beam at the laser source can be properly described by the (hopefully

Living Reviews in Relativity

http://www. livingreviews.org//rr-2010-1 


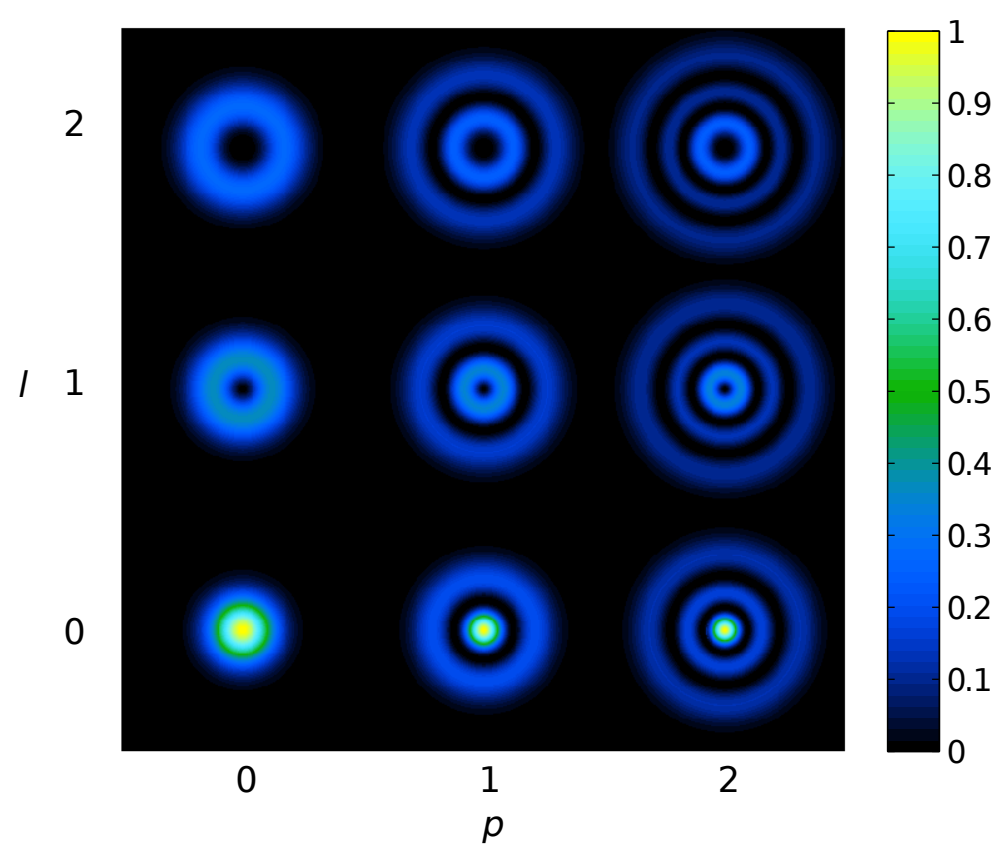

Figure 46: Intensity profiles for helical Laguerre-Gauss modes $u_{p l}$. The $u_{00}$ mode is identical to the Hermite-Gauss mode of order 0. Higher-order modes show a widening of the intensity and decreasing peak intensity. The number of concentric dark rings is given by the radial mode index $p$.

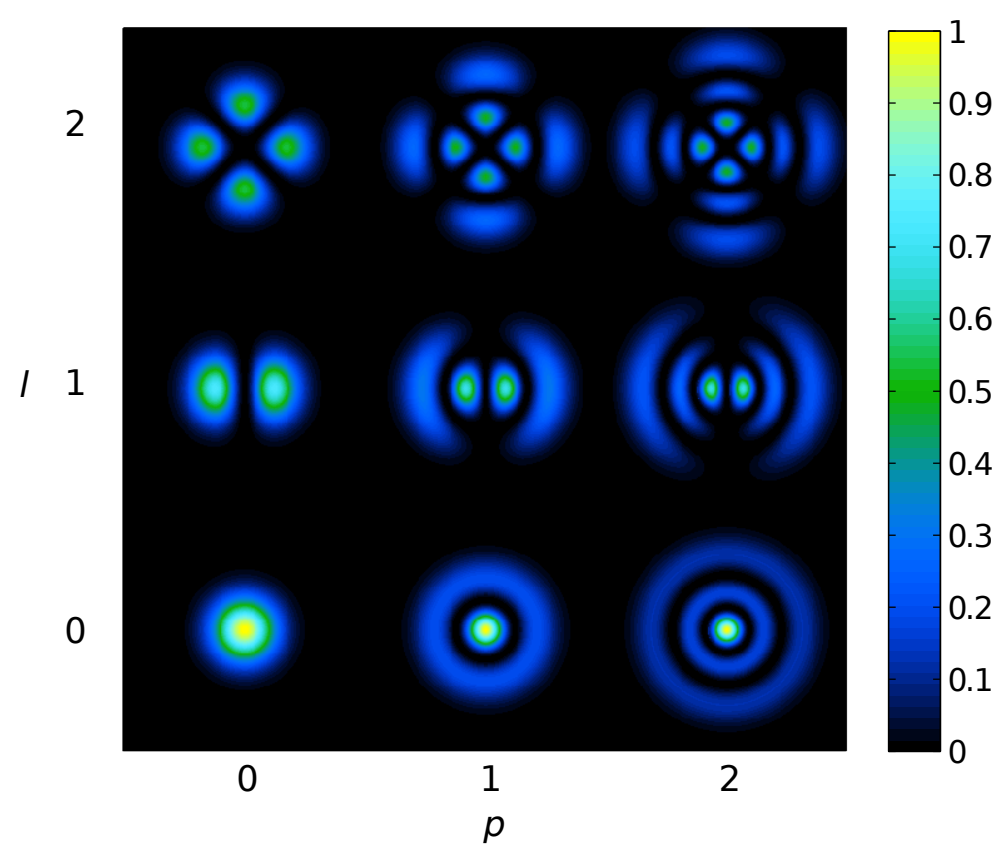

Figure 47: Intensity profiles for sinusoidal Laguerre-Gauss modes $u_{p l}^{\text {alt }}$. The $u_{p 0}$ modes are identical to the helical modes. However, for azimuthal mode indices $l>0$ the pattern shows $l$ dark radial lines in addition to the $p$ dark concentric rings. 
known) beam parameter of the laser's output mode. In addition, in most stable cavities the light fields should be described by using the respective cavity eigenmodes. Then, the remaining beam parameters can be computed by tracing the beam through the optical system. 'Trace' in this context means that a beam starting at a location with an already-known beam parameter is propagated mathematically through the optical system. At every optical element along the path the beam parameter is transformed according to the ABCD matrix of the element (see below).

\subsection{ABCD matrices}

The transformation of the beam parameter can be performed by the ABCD matrix-formalism [34, 50]. When a beam passes an optical element or freely propagates though space, the initial beam parameter $q_{1}$ is transformed into $q_{2}$. This transformation can be described by four real coefficients as follows:

$$
\frac{q_{2}}{n_{2}}=\frac{A \frac{q_{1}}{n_{1}}+B}{C \frac{q_{1}}{n_{1}}+D}
$$

with the coefficient matrix

$$
M=\left(\begin{array}{ll}
A & B \\
C & D
\end{array}\right),
$$

$n_{1}$ being the index of refraction at the beam segment defined by $q_{1}$, and $n_{2}$ the index of refraction at the beam segment described by $q_{2}$. ABCD matrices for some common optical components are given below, for the sagittal and tangential plane.

\section{Transmission through a mirror:}

A mirror in this context is a single, partly-reflecting surface with an angle of incidence of $90^{\circ}$. The transmission is described by

$$
M=\left(\begin{array}{cc}
1 & 0 \\
\frac{n_{2}-n_{1}}{R_{\mathrm{C}}} & 1
\end{array}\right)
$$

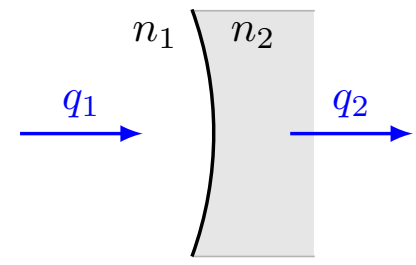

Figure 48

with $R_{\mathrm{C}}$ being the radius of curvature of the spherical surface. The sign of the radius is defined such that $R_{\mathrm{C}}$ is negative if the centre of the sphere is located in the direction of propagation. The curvature shown above (in Figure 48), for example, is described by a positive radius. The matrix for the transmission in the opposite direction of propagation is identical. 


\section{Reflection at a mirror:}

The matrix for reflection is given by

$$
M=\left(\begin{array}{cc}
1 & 0 \\
-\frac{2 n_{1}}{R_{\mathrm{C}}} & 1
\end{array}\right)
$$

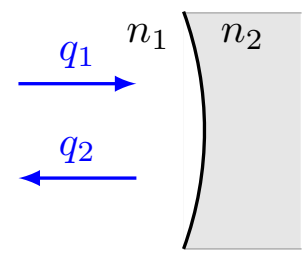

\section{Figure 49}

The reflection at the back surface can be described by the same type of matrix by setting $C=$ $2 n_{2} / R_{\mathrm{C}}$.

\section{Transmission through a beam splitter:}

A beam splitter is understood as a single surface with an arbitrary angle of incidence $\alpha_{1}$. The matrices for transmission and reflection are different for the sagittal and tangential planes $\left(M_{\mathrm{S}}\right.$ and $\left.M_{\mathrm{t}}\right)$ :

$$
\begin{aligned}
& M_{\mathrm{t}}=\left(\begin{array}{cc}
\frac{\cos \alpha_{2}}{\cos \alpha_{1}} & 0 \\
\frac{\Delta n}{R_{\mathrm{C}}} & \frac{\cos \alpha_{1}}{\cos \alpha_{2}}
\end{array}\right) \\
& M_{\mathrm{S}}=\left(\begin{array}{cc}
1 & 0 \\
\frac{\Delta n}{R_{\mathrm{C}}} & 1
\end{array}\right)
\end{aligned}
$$

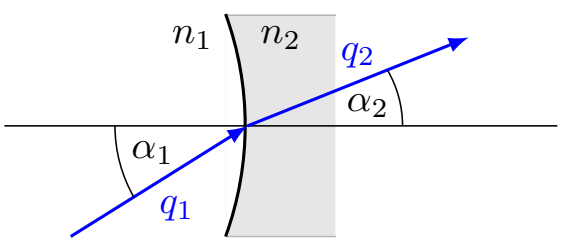

Figure 50

with $\alpha_{2}$ given by Snell's law:

$$
n_{1} \sin \left(\alpha_{1}\right)=n_{2} \sin \left(\alpha_{2}\right),
$$

and $\Delta n$ by

$$
\Delta n=\frac{n_{2} \cos \left(\alpha_{2}\right)-n_{1} \cos \left(\alpha_{1}\right)}{\cos \left(\alpha_{1}\right) \cos \left(\alpha_{2}\right)}
$$

If the direction of propagation is reversed, the matrix for the sagittal plane is identical and the matrix for the tangential plane can be obtained by changing the coefficients A and D as follows:

$$
\begin{aligned}
& A \longrightarrow 1 / A, \\
& D \longrightarrow 1 / D .
\end{aligned}
$$




\section{Reflection at a beam splitter:}

The reflection at the front surface of a beam splitter is given by:

$$
\begin{aligned}
& M_{\mathrm{t}}=\left(\begin{array}{cc}
1 & 0 \\
-\frac{2 n_{1}}{R_{\mathrm{C}} \cos \alpha_{1}} & 1
\end{array}\right) \\
& M_{\mathrm{s}}=\left(\begin{array}{cc}
1 & 0 \\
-\frac{2 n_{1} \cos \alpha_{1}}{R_{\mathrm{C}}} & 1
\end{array}\right)
\end{aligned}
$$

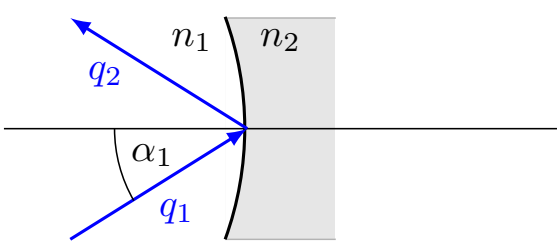

Figure 51

To describe a reflection at the back surface the matrices have to be changed as follows:

$$
\begin{aligned}
& R_{\mathrm{C}} \longrightarrow-R_{\mathrm{C}}, \\
& n_{1} \longrightarrow n_{2}, \\
& \alpha_{1} \longrightarrow-\alpha_{2} .
\end{aligned}
$$

\section{Transmission through a thin lens:}

A thin lens transforms the beam parameter as follows:

$$
M=\left(\begin{array}{cc}
1 & 0 \\
-\frac{1}{f} & 1
\end{array}\right)
$$

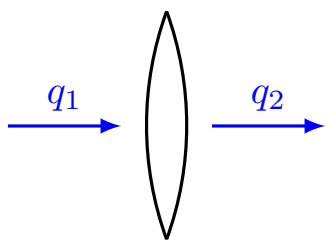

Figure 52

where $f$ is the focal length. The matrix for the opposite direction of propagation is identical. Here it is assumed that the thin lens is surrounded by 'spaces' with index of refraction $n=1$.

\section{Transmission through a free space:}

As mentioned above, the beam in free space can be described by one base parameter $q_{0}$. In some cases it is convenient to use a matrix similar to that used for the other components to describe the $z$-dependency of $q(z)=q_{0}+z$. On propagation through a free space of the length $L$ and index of refraction $n$, the beam parameter is transformed as follows.

$$
M=\left(\begin{array}{cc}
1 & \frac{L}{n} \\
0 & 1
\end{array}\right)
$$

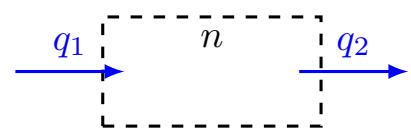

Figure 53

The matrix for the opposite direction of propagation is identical. 


\section{Interferometer Matrix with Hermite-Gauss Modes}

In the plane-wave analysis Section 1.4, a laser beam is described in general by the sum of various frequency components of its electric field

$$
E(t, z)=\sum_{j} a_{j} \exp \left(\mathrm{i}\left(\omega_{j} t-k_{j} z\right)\right) .
$$

Here we include the geometric shape of the beam by describing each frequency component as a sum of Hermite-Gauss modes:

$$
E(t, x, y, z)=\sum_{j} \sum_{n, m} a_{j n m} u_{n m}(x, y) \exp \left(\mathrm{i}\left(\omega_{j} t-k_{j} z\right)\right) .
$$

The shape of such a beam does not change along the $z$-axis (in the paraxial approximation). More precisely, the spot size and the position of the maximum intensity with respect to the $z$-axis may change, but the relative intensity distribution across the beam does not change its shape. Each part of the sum may be treated as an independent field that can be described using the equation for plane-waves with only two exceptions:

- the propagation through free space has to include the Gouy phase shift, and

- upon reflection or transmission at a mirror or beam splitter the different Hermite-Gauss modes may be coupled (see below).

The Gouy phase shift can be included in the simulation in several ways. For example, for reasons of flexibility the Gouy phase has been included in FINESSE as a phase shift of the component space.

\subsection{Coupling of Hermite-Gauss modes}

Let us consider two different cavities with different sets of eigenmodes. The first set is characterised by the beam parameter $q_{1}$ and the second by the parameter $q_{2}$. A beam with all power in the fundamental mode $u_{00}\left(q_{1}\right)$ leaves the first cavity and is injected into the second. Here, two 'misconfigurations' are possible:

- if the optical axes of the beam and the second cavity do not overlap perfectly, the setup is called misaligned,

- if the beam size or shape at the second cavity does not match the beam shape and size of the (resonant) fundamental eigenmode $\left(q_{1}\left(z_{\text {cav }}\right) \neq q_{2}\left(z_{\text {cav }}\right)\right)$, the beam is then not mode-matched to the second cavity, i.e., there is a mode mismatch.

The above misconfigurations can be used in the context of simple beam segments. We consider the case in which the beam parameter for the input light is specified. Ideally, the ABCD matrices then allow one to trace a beam through the optical system by computing the proper beam parameter for each beam segment. In this case, the basis system of Hermite-Gauss modes is transformed in the same way as the beam, so that the modes are not coupled.

For example, an input beam described by the beam parameter $q_{1}$ is passed through several optical components, and at each component the beam parameter is transformed according to the respective ABCD matrix. Thus, the electric field in each beam segment is described by HermiteGauss modes based on different beam parameters, but the relative power between the HermiteGauss modes with different mode numbers remains constant, i.e., a beam in a $u_{00}$ mode is described as a pure $u_{00}$ mode throughout the entire system. 
In practice, it is usually impossible to compute proper beam parameter for each beam segment as suggested above, especially when the beam passes a certain segment more than once. A simple case that illustrates this point is reflection at a spherical mirror. Let the input beam be described by $q_{1}$. From Figure 49 we know that the proper beam parameter of the reflected beam is

$$
q_{2}=\frac{q_{1}}{-2 q_{1} / R_{\mathrm{C}}+1}
$$

with $R_{\mathrm{C}}$ being the radius of curvature of the mirror. In general, we get $q_{1} \neq q_{2}$ and thus two different 'proper' beam parameters for the same beam segment. Only one special radius of curvature would result in matched beam parameters $\left(q_{1}=q_{2}\right)$.

\subsection{Coupling coefficients for Hermite-Gauss modes}

Hermite-Gauss modes are coupled whenever a beam is not matched to a cavity or to a beam segment or if the beam and the segment are misaligned. This coupling is sometimes referred to as 'scattering into higher-order modes' because in most cases the laser beam is a considered as a pure $\mathrm{TEM}_{00}$ mode and any mode coupling would transfer power from the fundamental into higher-order modes. However, in general, every mode with non-zero power will transfer energy into other modes whenever mismatch or misalignment occur, and this effect also includes the transfer from higher orders into a low order.

To compute the amount of coupling the beam must be projected into the base system of the cavity or beam segment it is being injected into. This is always possible, provided that the paraxial approximation holds, because each set of Hermite-Gauss modes, defined by the beam parameter at a position $z$, forms a complete set. Such a change of the basis system results in a different distribution of light power in the new Hermite-Gauss modes and can be expressed by coupling coefficients that yield the change in the light amplitude and phase with respect to mode number.

Let us assume that a beam described by the beam parameter $q_{1}$ is injected into a segment described by the parameter $q_{2}$. Let the optical axis of the beam be misaligned: the coordinate system of the beam is given by $(x, y, z)$ and the beam travels along the $z$-axis. The beam segment is parallel to the $z^{\prime}$-axis and the coordinate system $\left(x^{\prime}, y^{\prime}, z^{\prime}\right)$ is given by rotating the $(x, y, z)$ system around the $y$-axis by the misalignment angle $\gamma$. The coupling coefficients are defined as

$$
u_{n m}\left(q_{1}\right) \exp (\mathrm{i}(\omega t-k z))=\sum_{n^{\prime}, m^{\prime}} k_{n, m, n^{\prime}, m^{\prime}} u_{n^{\prime} m^{\prime}}\left(q_{2}\right) \exp \left(\mathrm{i}\left(\omega t-k z^{\prime}\right)\right),
$$

where $u_{n m}\left(q_{1}\right)$ are the Hermite-Gauss modes used to describe the injected beam and $u_{n^{\prime} m^{\prime}}\left(q_{2}\right)$ are the 'new' modes that are used to describe the light in the beam segment. Note that including the plane wave phase propagation within the definition of coupling coefficients is very important because it results in coupling coefficients that are independent of the position on the optical axis for which the coupling coefficients are computed.

Using the fact that the Hermite-Gauss modes $u_{n m}$ are orthonormal, we can compute the coupling coefficients by the convolution [6]

$$
k_{n, m, n^{\prime}, m^{\prime}}=\exp \left(\mathrm{i} 2 k z^{\prime} \sin ^{2}\left(\frac{\gamma}{2}\right)\right) \iint d x^{\prime} d y^{\prime} u_{n^{\prime} m^{\prime}} \exp \left(\mathrm{i} k x^{\prime} \sin \gamma\right) u_{n m}^{*} .
$$

Since the Hermite-Gauss modes can be separated with respect to $x$ and $y$, the coupling coefficients can also be split into $k_{n m n^{\prime} m^{\prime}}=k_{n n^{\prime}} k_{m m^{\prime}}$. These equations are very useful in the paraxial approximation as the coupling coefficients decrease with large mode numbers. In order to be described as paraxial, the angle $\gamma$ must not be larger than the diffraction angle. In addition, to obtain correct results with a finite number of modes the beam parameters $q_{1}$ and $q_{2}$ must not differ too much.

Living Reviews in Relativity

http: //www. livingreviews.org/lrr-2010-1 
The convolution given in Equation (164) can be computed directly using numerical integration. However, this is computationally very expensive. The following is based on the work of BayerHelms [6]. Another very good description of coupling coefficients and their derivation can be found in the work of Vinet [55]. In [6] the above projection integral is partly solved and the coupling coefficients are given by simple sums as functions of $\gamma$ and the mode mismatch parameter $K$, which are defined by

$$
K=\frac{1}{2}\left(K_{0}+\mathrm{i} K_{2}\right)
$$

where $K_{0}=\left(z_{R}-z_{R}^{\prime}\right) / z_{R}^{\prime}$ and $K_{2}=\left(\left(z-z_{0}\right)-\left(z^{\prime}-z_{0}^{\prime}\right)\right) / z_{R}^{\prime}$. This can also be written using $q=\mathrm{i} z_{\mathrm{R}}+z-z_{0}$, as

$$
K=\frac{\mathrm{i}\left(q-q^{\prime}\right)^{*}}{2 \Im\left\{q^{\prime}\right\}} .
$$

The coupling coefficients for misalignment and mismatch (but no lateral displacement) can then be written as

$$
k_{n n^{\prime}}=(-1)^{n^{\prime}} E^{(x)}\left(n ! n^{\prime} !\right)^{1 / 2}\left(1+K_{0}\right)^{n / 2+1 / 4}\left(1+K^{*}\right)^{-\left(n+n^{\prime}+1\right) / 2}\left\{S_{g}-S_{u}\right\}
$$

where

$$
\begin{aligned}
S_{g} & =\sum_{\mu=0}^{[n / 2]} \sum_{\mu^{\prime}=0}^{\left[n^{\prime} / 2\right]} \frac{(-1)^{\mu} \bar{X}^{n-2 \mu} X^{n^{\prime}-2 \mu^{\prime}}}{(n-2 \mu) !\left(n^{\prime}-2 \mu^{\prime}\right) !} \sum_{\sigma=0}^{\min \left(\mu, \mu^{\prime}\right)} \frac{(-1)^{\sigma} \bar{F}^{\mu-\sigma} F^{\mu^{\prime}-\sigma}}{a}(2 \sigma) !(\mu-\sigma) !\left(\mu^{\prime}-\sigma\right) !, \\
S_{u} & =\sum_{\mu=0}^{[(n-1) / 2]} \sum_{\mu^{\prime}=0}^{\left[\left(n^{\prime}-1\right) / 2\right]} \frac{(-1)^{\mu} \bar{X}^{n-2 \mu-1} X^{n^{\prime}-2 \mu^{\prime}-1}}{(n-2 \mu-1) !\left(n^{\prime}-2 \mu^{\prime}-1\right) !} \sum_{\sigma=0}^{\min \left(\mu, \mu^{\prime}\right)} \frac{(-1)^{\sigma} \bar{F}^{\mu-\sigma} F^{\mu^{\prime}-\sigma}}{(2 \sigma+1) !(\mu-\sigma) !\left(\mu^{\prime}-\sigma\right) !} .
\end{aligned}
$$

The corresponding formula for $k_{m m^{\prime}}$ can be obtained by replacing the following parameters: $n \rightarrow$ $m, n^{\prime} \rightarrow m^{\prime}, X, \bar{X} \rightarrow 0$ and $E^{(x)} \rightarrow 1$ (see below). The notation $[n / 2]$ means

$$
\left[\frac{m}{2}\right]= \begin{cases}m / 2 & \text { if } m \text { is even } \\ (m-1) / 2 & \text { if } m \text { is odd. }\end{cases}
$$

The other abbreviations used in the above definition are

$$
\begin{aligned}
& \bar{X}=\left(\mathrm{i} z_{\mathrm{R}}^{\prime}-z^{\prime}\right) \sin (\gamma) /\left(\sqrt{1+K^{*}} w_{0}\right), \\
& X=\left(\mathrm{i} z_{\mathrm{R}}+z^{\prime}\right) \sin (\gamma) /\left(\sqrt{1+K^{*}} w_{0}\right), \\
& F=K /\left(2\left(1+K_{0}\right)\right), \\
& \bar{F}=K^{*} / 2 \\
& E^{(x)}=\exp \left(-\frac{X \bar{X}}{2}\right) .
\end{aligned}
$$

In general, the Gaussian beam parameter might be different for the sagittal and tangential planes and a misalignment can be given for both possible axes (around the $y$-axis and around the $x$-axis), in this case the coupling coefficients are given by

$$
k_{n m m^{\prime} n^{\prime}}=k_{n n^{\prime}} k_{m m^{\prime}}
$$

where $k_{n n^{\prime}}$ is given above with

$$
\begin{aligned}
& q \rightarrow q_{t} \\
& \text { and } \\
& w_{0} \rightarrow w_{t, 0}, \text { etc. }
\end{aligned}
$$


and $\gamma \rightarrow \gamma_{y}$ is a rotation about the $y$-axis. The $k_{m m^{\prime}}$ can be obtained with the same formula, with the following substitutions:

$$
\begin{aligned}
& n \rightarrow m, \\
& n^{\prime} \rightarrow m^{\prime}, \\
& q \rightarrow q_{s}, \\
& \text { thus } \\
& w_{0} \rightarrow w_{s, 0}, \text { etc. }
\end{aligned}
$$

and $\gamma \rightarrow \gamma_{x}$ is a rotation about the $x$-axis.

At each component a matrix of coupling coefficients has to be computed for transmission and reflection; see Figure 54.

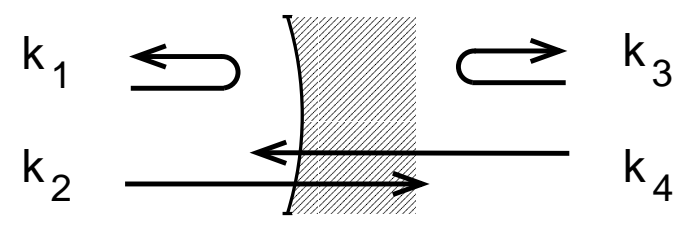

Figure 54: Coupling coefficients for Hermite-Gauss modes: for each optical element and each direction of propagation complex coefficients $k$ for transmission and reflection have to be computed. In this figure $k_{1}, k_{2}, k_{3}, k_{4}$ each represent a matrix of coefficients $k_{n m n^{\prime} m^{\prime}}$ describing the coupling of $u_{n, m}$ into $u_{n^{\prime}, m^{\prime}}$. 

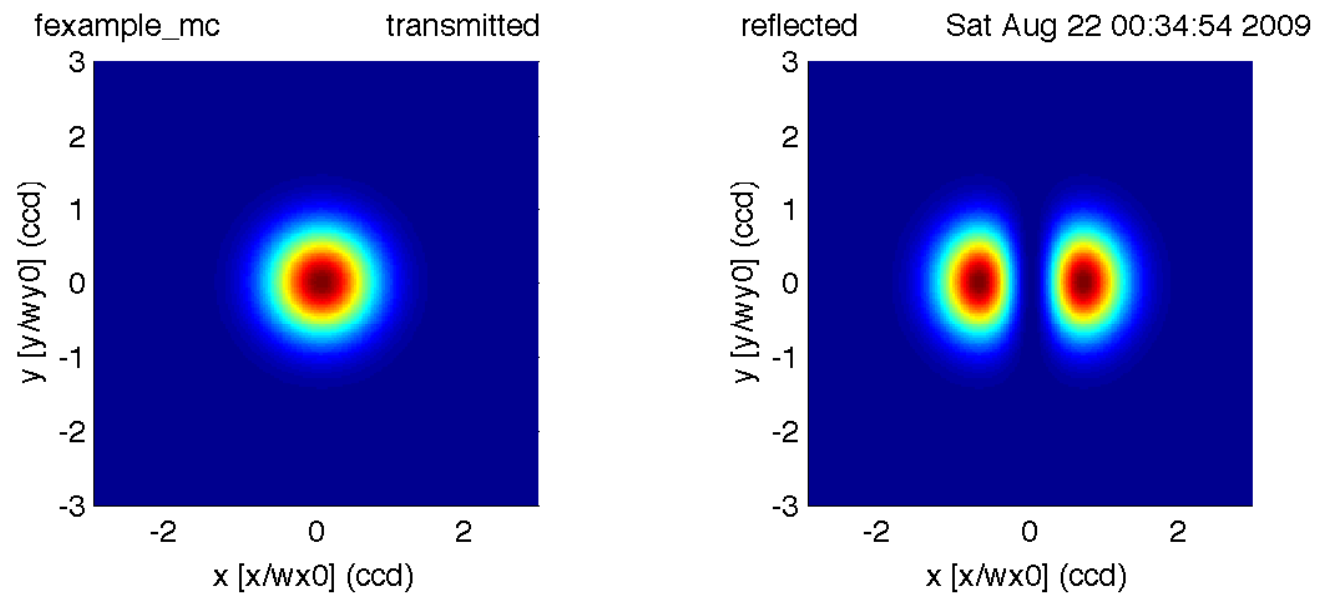

Figure 56: FINESSE example: Mode cleaner

cav cav1 bs1 n3 bs1 n4 run1: beam ccd ntrans run2: beam ccd nrefl

xaxis ccd x lin -33200

$\mathrm{x} 2$ axis ccd y lin -33200

yaxis abs
$\%$ computing cavity parameters

$\%$ beam shape in transmission

$\%$ beam shape in reflection

$\%$ tuning $\mathrm{x}, \mathrm{y}$ axes of beam detector

$\%$ plotting the absolute intensity

\subsubsection{LG33 mode}
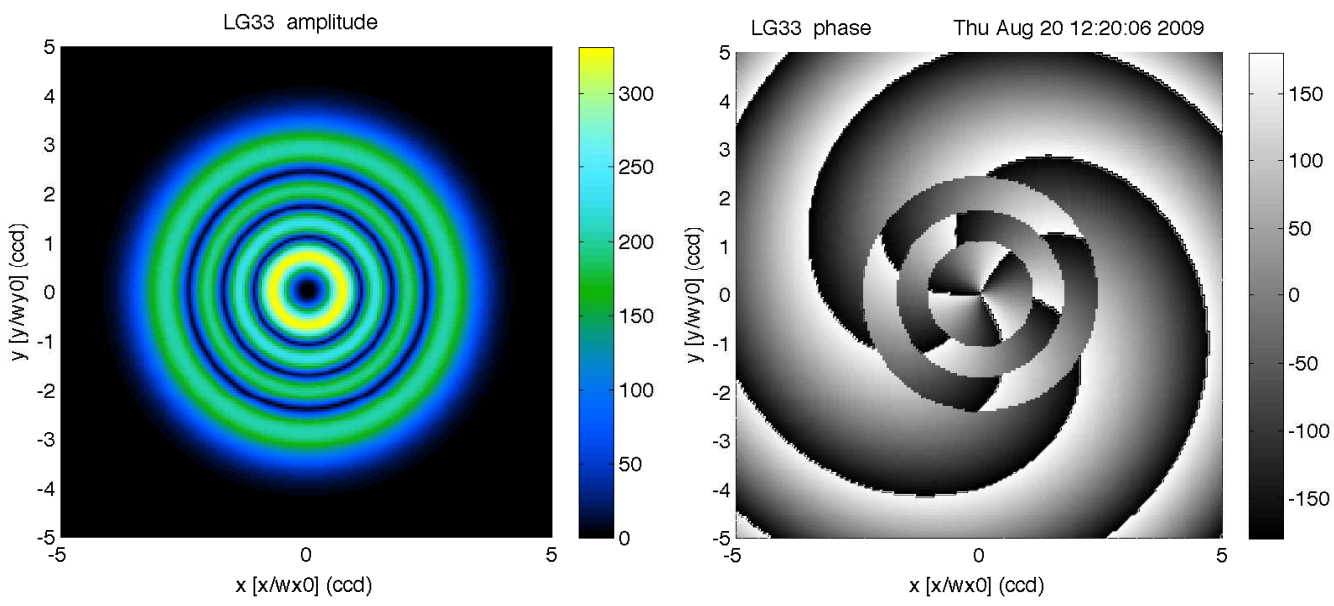

Figure 57: Finesse example: LG33 mode. The ring structure in the phase plot is due to phase jumps, which could be removed by applying a phase 'unwrap'.

Finesse uses the Hermite-Gauss modes as a base system for describing the spatial properties of laser beams. However, Laguerre-Gauss modes can be created using the coefficients given in Equation (149). This example demonstrates this and the use of a 'beam' detector to plot amplitude and phase of a beam cross section. 


\section{Finesse input file for 'LG33 mode'}

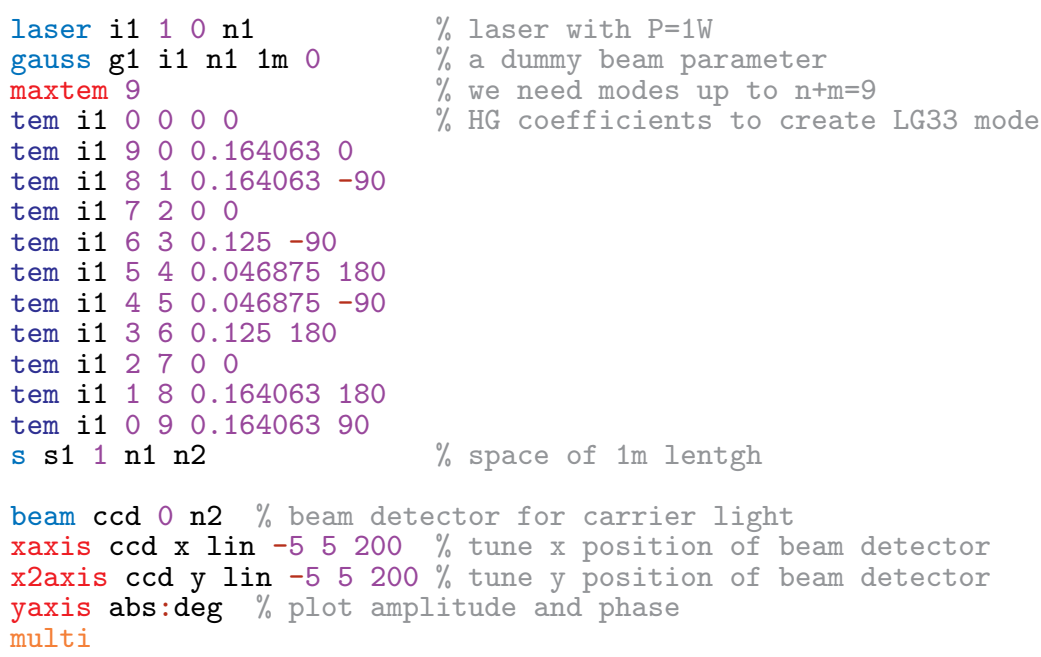




\section{Acknowledgements}

We would like to thank our colleagues in the GEO 600 project for many useful discussions over the years. AF acknowledges support from the University of Birmingham. KS acknowledges support from the University of Glasgow and the Albert Einstein Institute, Hannover. Some of the illustrations have been prepared using the component library by Alexander Franzen.

\section{A The Interferometer Simulation Finesse}

Throughout this document we have provided a number of text files that can be used as input files for the interferometer simulation FINESSE [19, 22]. FINESSE is a numerical simulation written in the $\mathrm{C}$ language; it is available free of charge for Linux, Windows and Macintosh computers and can be obtained online: http://www.gwoptics.org/finesse/.

FINESSE provides a fast and versatile tool that has proven to be very useful during the design and commissioning of interferometric gravitational-wave detectors. However, the program has been designed to allow the analysis of arbitrary, user-defined optical setups. In addition, it is easy to install and use. Therefore FinEsse is well suited to study basic optical properties, such as, the power enhancement in a resonating cavity and modulation-demodulation methods.

We encourage the reader to obtain FINESSE and to learn its basic usage by running the included example files (and by making use of its extensive manual). The FinEsse input files provided in this article are in most cases very simple and illustrate single concepts in interferometry. We believe that even a FinESSE novice should be able to use them as starting points to play and explore freely, for example by changing parameters, or by adding further optical components. This type of 'numerical experimentation' can provide insights similar to real experiments, supplementing the understanding through a mathematical analysis with experience and intuitions. 


\section{References}

[1] Abramochkin, E., and Volostnikov, V., "Beam transformations and nontransformed beams", Opt. Commun., 83, 123-135, (1991). [ADS]. (Cited on page 64.)

[2] Abramowitz, M., and Stegun, I.A., eds., Handbook of Mathematical Functions with Formulas, Graphs, and Mathematical Tables, (Dover, New York, 1965), corr. edition. [Google Books]. (Cited on page 23.)

[3] Acernese, F. (Virgo Collaboration), "The Virgo automatic alignment system", Class. Quantum Grav., 23(8), S91-S101, (2006). [DOI]. (Cited on page 6.)

[4] Acernese, F. (Virgo Collaboration), Advanced Virgo Baseline Design, VIR-027A-09, (Virgo, Cascina, 2009). Related online version (cited on 22 September 2009): http://www.virgo.infn.it/advirgo/docs.html. (Cited on page 5.)

[5] Advanced LIGO Reference Design, LIGO M060056-08-M, (LIGO, Pasadena, CA, 2007). Related online version (cited on 20 September 2009):

http://www.ligo.caltech.edu/docs/M/M060056-08/M060056-08.pdf. (Cited on page 5.)

[6] Bayer-Helms, F., "Coupling coefficients of an incident wave and the modes of spherical optical resonator in the case of mismatching and misalignment", Appl. Optics, 23, 1369-1380, (1984). [DOI]. (Cited on pages 70 and 71.)

[7] Beijersbergen, M.W., Allen, L., van der Veen, H.E.L.O., and Woerdman, J.P., "Astigmatic laser mode converters and transfer of orbital angular momentum", Opt. Commun., 96, 123132, (1993). [DOI]. (Cited on page 64.)

[8] Boyd, R.W., "Intuitive explanation of the phase anomaly of focused light beams", J. Opt. Soc. Am., 70(7), 877-880, (1980). (Cited on page 62.)

[9] Braginsky, V.B., Gorodetsky, M.L., Khalili, F.Y., and Thorne, K.S., "Energetic quantum limit in large-scale interferometers", in Meshkov, S., ed., Gravitational Waves: Third Edoardo Amaldi Conference, Pasadena, California, 12-16 July, 1999, AIP Conference Proceedings, vol. 523, pp. 180-190, (American Institute of Physics, Melville, NY, 2000). [DOI], [ADS], [arXiv:gr-qc/9907057]. (Cited on page 7.)

[10] Caves, C.M., "Quantum-mechanical noise in an interferometer", Phys. Rev. D, 23, 1693-1708, (1981). [DOI]. (Cited on page 7.)

[11] Chelkowski, S., Squeezed Light and Laser Interferometric Gravitational Wave Detectors, Ph.D. Thesis, (Universität Hannover, Hannover, 2007). Related online version (cited on 28 September 2009):

http://edok01.tib.uni-hannover.de/edoks/e01dh07/537859527.pdf. (Cited on page 27.)

[12] Chelkowski, S., Hild, S., and Freise, A., "Prospects of higher-order Laguerre-Gauss modes in future gravitational wave detectors", Phys. Rev. D, 79, 122002, 1-11, (2009). [DOI], [arXiv:0901.4931 [gr-qc]]. (Cited on page 62.)

[13] Davis, T.A., Direct Methods for Sparse Linear Systems, Fundamentals of Algorithms, vol. 2, (SIAM, Philadelphia, 2006). Related online version (cited on 28 September 2009): http://www.cise.ufl.edu/research/sparse/CSparse/. (Cited on page 12.) 
[14] Drever, R.W.P., Hall, J.L., Kowalski, F.V., Hough, J., Ford, G.M., Munley, A.J., and Ward, H., "Laser phase and frequency stabilization using an optical resonator", Appl. Phys. B, 31, 97-105, (1983). [DOI]. (Cited on page 47.)

[15] Drever, R.W.P., Hough, J., Munley, A.J., Lee, S.-A., Spero, R.E., Whitcomb, S.E., Ward, H., Ford, G.M., Hereld, M., Robertson, N.A., Kerr, I., Pugh, J.R., Newton, G.P., Meers, B.J., Brooks III, E.D., and Gürsel, Y., "Gravitational wave detectors using laser interferometers and optical cavities: Ideas, principles and prospects", in Meystre, P., and Scully, M.O., eds., Quantum Optics, Experimental Gravity, and Measurement Theory, Proceedings of the NATO Advanced Study Institute, held August 16-29, 1981 in Bad Windsheim, Germany, NATO ASI Series B, vol. 94, pp. 503-514, (Plenum Press, New York, 1983). (Cited on page 6.)

[16] Fabry, C., and Pérot, A., "Théorie et applications d'une nouvelle méthode de spectroscopie interférentielle", Ann. Chim. Phys., 16, 115-144, (1899). (Cited on pages 6 and 36.)

[17] Fattaccioli, D., Boulharts, A., Brillet, A., and Man, C.N., "Sensitivity of multipass and FabryPerot delay lines to small misalignments", J. Optics (Paris), 17(3), 115-127, (1986). [DOI]. (Cited on page 6.)

[18] Forward, R.L., "Wideband laser-interferometer gravitational-radiation experiment", Phys. Rev. D, 17, 379-390, (1978). [DOI]. (Cited on pages 6 and 36.)

[19] Freise, A., "FINESSE: An Interferometer Simulation", personal homepage, Andreas Freise. URL (cited on 16 January 2010):

http://www.gwoptics.org/finesse. (Cited on pages 5 and 76.)

[20] Freise, A., The Next Generation of Interferometry: Multi-Frequency Optical Modelling, Control Concepts and Implementation, Ph.D. Thesis, (Universität Hannover, Hannover, 2003). Related online version (cited on 28 September 2009):

http://edok01.tib.uni-hannover.de/edoks/e01dh03/361006918.pdf. （Cited on page 33.)

[21] Freise, A., Bunkowski, A., and Schnabel, R., "Phase and alignment noise in grating interferometers", New J. Phys., 9, 433, (2007). [DOI], [arXiv:0711.0291]. URL (cited on 17 January 2010): http://stacks.iop.org/1367-2630/9/433. (Cited on page 6.)

[22] Freise, A., Heinzel, G., Lück, H., Schilling, R., Willke, B., and Danzmann, K., "Frequencydomain interferometer simulation with higher-order spatial modes", Class. Quantum Grav., 21(5), S1067-S1074, (2004). [DOI], [arXiv:gr-qc/0309012]. (Cited on pages 5 and 76.)

[23] Fritschel, P., "Second generation instruments for the Laser Interferometer Gravitational Wave Observatory (LIGO)", in Cruise, M., and Saulson, P., eds., Gravitational-Wave Detection, Waikoloa, HI, USA, 23 August 2002, Proc. SPIE, vol. 4856, pp. 282-291, (SPIE, Bellingham, WA, 2003). [DOI], [gr-qc/0308090]. (Cited on page 5.)

[24] Giovannetti, V., Lloyd, S., and Maccone, L., "Quantum-Enhanced Measurements: Beating the Standard Quantum Limit", Science, 306, 1330-1336, (2004). [DOI], [ADS], [arXiv:quantph/0412078]. (Cited on page 7.)

[25] Gouy, L.G., "Sur la propagation anomale des ondes", C. R. Acad. Sci., 111, 33, (1890). (Cited on page 62.)

[26] Gouy, L.G., "Sur une propriété nouvelle des ondes lumineuses", C. R. Acad. Sci., 110, 1251, (1890). (Cited on page 62.) 
[27] Gradshteyn, I.S., and Ryzhik, I.M., Tables of Integrals, Series, and Products, (Academic Press, San Diego; London, 1994), 5th edition. (Cited on page 23.)

[28] Hecht, E., Optics, (Addison-Wesley, Reading, MA, 2002), 4th edition. (Cited on page 13.)

[29] Heinzel, G., Advanced optical techniques for laser-interferometric gravitational-wave detectors, Ph.D. Thesis, (Universität Hannover, Hannover, 1999). Related online version (cited on 28 September 2009):

http://edok01.tib.uni-hannover.de/edoks/e002/265099560.pdf. (Cited on pages 17 and 24.)

[30] Heinzel, G., Strain, K. A., Mizuno, J., Skeldon, K. D., Willke, B., Winkler, W., Schilling, R., Rüdiger, A., and Danzmann, K., "Experimental Demonstration of a Suspended Dual Recycling Interferometer for Gravitational Wave Detection", Phys. Rev. Lett., 81, 5493-5496, (1998). [DOI]. (Cited on page 6.)

[31] Herriott, D., Kogelnik, H., and Kompfner, R., "Off-Axis Paths in Spherical Mirror Interferometers", Appl. Optics, 3, 523-526, (1964). [DOI], [ADS]. (Cited on page 6.)

[32] Jaekel, M.T., and Reynaud, S., "Quantum Limits in Interferometric Measurements", Europhys. Lett., 13, 301-306, (1990). [DOI], [quant-ph/0101104]. (Cited on page 7.)

[33] Kenyon, I.R., The Light Fantastic: A Modern Introduction to Classical and Quantum Optics, (Oxford University Press, Oxford; New York, 2008). [Google Books]. (Cited on page 13.)

[34] Kogelnik, H., "On the Propagation of Gaussian Beams of Light Through Lenslike Media Including those with a Loss or Gain Variation", Appl. Optics, 4(12), 1562-1569, (1965). [ADS]. (Cited on pages 58 and 66.)

[35] Kogelnik, H., and Li, T., "Laser Beams and Resonators", Proc. IEEE, 54, 1312-1329, (1966). (Cited on pages 53 and 57.)

[36] Kuroda, K. (LCGT Collaboration), "The status of LCGT", Class. Quantum Grav., 23(8), S215-S221, (2006). [DOI]. (Cited on page 5.)

[37] Lax, M., Louisell, W.H., and McKnight, W.B., "From Maxwell to paraxial wave optics", Phys. Rev. A, 11, 1365-1370, (1975). [DOI]. (Cited on page 53.)

[38] Loudon, R., and Knight, P.L., "Squeezed Light", J. Mod. Opt., 34, 709-759, (1987). [DOI], [ADS]. (Cited on page 7.)

[39] Malec, M., Commissioning of advanced, dual-recycled gravitational-wave detectors: simulations of complex optical systems guided by the phasor picture, Ph.D. Thesis, (Universität Hannover, Hannover, 2006). Related online version (cited on 28 September 2009):

http://edok01.tib.uni-hannover.de/edoks/e01dh06/510301622.pdf. (Cited on page 27.)

[40] Matuschek, N., Kartner, F.X., and Keller, U., "Exact coupled-mode theories for multilayer interference coatings with arbitrary strong index modulations", IEEE J. Quantum Electron., 33, 295-302, (1997). [DOI]. (Cited on page 13.)

[41] Meers, B.J., "Recycling in laser-interferometric gravitational-wave detectors", Phys. Rev. D, 38, 2317-2326, (1988). [DOI]. (Cited on page 6.) 
[42] Michelson, A.A., and Morley, E.W., "On the Relative Motion of the Earth and the Luminiferous Ether", Am. J. Sci., 34, 333-345, (1887). Related online version (cited on 20 September 2009):

http://www.aip.org/history/gap/PDF/michelson.pdf. (Cited on page 36.)

[43] Mizuno, J., and Yamaguchi, I., "Method for analyzing multiple-mirror coupled optical systems", J. Opt. Soc. Am. A, 16, 1730-1739, (1999). [DOI]. (Cited on page 13.)

[44] Morrison, E., Meers, B.J., Robertson, D.I., and Ward, H., "Automatic alignment of optical interferometers", Appl. Optics, 33, 5041-5049, (1994). [DOI]. (Cited on page 6.)

[45] Newport Catalogue, (Newport Corporation, Irvine, CA, 2008). Related online version (cited on 20 September 2009):

http://www. newport.com/. (Cited on page 14.)

[46] Rigrod, W.W., "The optical ring resonator", Bell Syst. Tech. J., 44, 907-916, (1965). (Cited on page 57.)

[47] Rowan, S., and Hough, J, "Gravitational Wave Detection by Interferometry (Ground and Space)", Living Rev. Relativity, 3, lrr-2000-3, (2000). URL (cited on 20 September 2009): http://www.livingreviews.org/lrr-2000-3. (Cited on page 5.)

[48] Rüdiger, A., "Phase relationship at a symmetric beamsplitter", unknown status, (1998). (Cited on page 16.)

[49] Saulson, P.R., Fundamentals of Interferometric Gravitational Wave Detectors, (World Scientific, Singapore; River Edge, NJ, 1994). (Cited on page 50.)

[50] Siegman, A.E., Lasers, (University Science Books, Sausalito, CA, 1986). [Google Books]. See also errata list at http://www.stanford.edu/\%7Esiegman/AES\%20LASERS\%20Book/. (Cited on pages $53,58,62$, and 66 .)

[51] Siegman, A.E., "Laser Beams and Resonators: Beyond the 1960s", IEEE J. Select. Topics Quantum Electron., 6, 1389-1399, (2000). [DOI]. Related online version (cited on 18 January 2010):

http://www.stanford.edu/ siegman/beams_and_resonators/beams_and_resonators_2. pdf. (Cited on page 53.)

[52] Siegman, A.E., "Laser Beams and Resonators: The 1960s", IEEE J. Select. Topics Quantum Electron., 6, 1380-1388, (2000). [DOI]. Related online version (cited on 18 January 2010): http://www.stanford.edu/ siegman/beams_and_resonators/beams_and_resonators_1. pdf. (Cited on page 53.)

[53] Vahlbruch, H., Chelkowski, S., Danzmann, K., and Schnabel, R., "Quantum engineering of squeezed states for quantum communication and metrology", New J. Phys., 9(10), 371, (2007). [DOI], [arXiv:0707.2845]. URL (cited on 17 January 2010):

http://stacks.iop.org/1367-2630/9/371. (Cited on page 7.)

[54] Vinet, J.-Y., "On Special Optical Modes and Thermal Issues in Advanced Gravitational Wave Interferometric Detectors", Living Rev. Relativity, 12, lrr-2009-5, (2009). URL (cited on 20 September 2009):

http://www.livingreviews.org/lrr-2009-5. (Cited on page 62.)

[55] Vinet, J.-Y. (Virgo Collaboration), The Virgo Physics Book, Vol. II: Optics and Related Topics, (Virgo, Cascina, 2001). URL (cited on 20 September 2009):

http://www.virgo.infn.it/vpb/. (Cited on pages 64 and 71.) 
[56] "Virgo", project homepage, Virgo Collaboration. URL (cited on 19 September 2009): http://www.virgo.infn.it/. (Cited on page 17.)

[57] Winkler, W., Danzmann, K., Grote, H., Hewitson, M., Hild, S., Hough, J., Lück, H., Malec, M., Freise, A., Mossavi, K., Rowan, S., Rüdiger, A., Schilling, R., Smith, J.R., Strain, K.A., Ward, H., and Willke, B., "The GEO 600 core optics", Opt. Commun., 280, 492-499, (2007). [DOI]. (Cited on page 6.)

[58] Yariv, A., Quantum Electronics, (J. Wiley \& Sons, New York, 1989), 3rd edition. (Cited on page 31.) 